\title{
GROTHENDIECK'S EXISTENCE THEOREM IN ANALYTIC GEOMETRY AND RELATED RESULTS
}

\author{
SIEGMUND KOSAREW
}

\begin{abstract}
We state and prove several kinds of analytification theorems of formal objects (such as coherent sheaves and formal complex spaces) which are in the spirit of Grothendieck's algebraization theorem in [EGA, III]. The formulation of the results was derived from deformation theory and especially M. Artin's work on representability of functors. The methods of proof depend heavily on a deeper study of cotangent complexes and resolvants. As applications one can deduce the convergence of formal versal deformations in diverse situations.
\end{abstract}

A powerful tool for the solution of many moduli problems in algebraic geometry is Grothendieck's existence theorem in [EGA, III, Theorème (5.1.4)]. This theorem gives a general algebraicity criterion for coherent formal sheaves and goes as follows.

Theorem (Grothendieck). Let $A$ be an adic noetherian ring, $Y=\operatorname{Spec}(A), \mathcal{I}$ an ideal of definition for $A, Y^{\prime}=V(\mathscr{I}), f: X \rightarrow Y$ a separated morphism of finite type and $X^{\prime}=f^{-1}\left(Y^{\prime}\right)$. Furthermore, let $\hat{Y}=Y_{/ Y^{\prime}}=\operatorname{Spf}(A), \hat{X}=X_{/ X^{\prime}}$ be the formal completions of $Y$ with respect to $Y^{\prime}$ and of $X$ with respect to $X^{\prime}$, and $\hat{f}: \hat{X} \rightarrow \hat{Y}$ the completion of $f$.

Then the functor $\mathscr{F} \mapsto \widehat{\mathscr{F}}$ gives an equivalence between the category of coherent $\mathscr{O}_{X}$-modules with proper support over $Y$ and the category of coherent $\mathscr{O}_{\widehat{X}}$-modules with proper support over $\hat{Y}$.

A generalization of this theorem to algebraic spaces (in the sense of Artin) can be found in [Knu, $\mathrm{V}, 6.3]$. The applications in algebraic deformation theory are usually made there where one has to verify the effectiveness of formal deformations (compare for instance [Art, §1]).

As the formulation of Grothendieck's theorem shows, a straightforward translation into the context of analytic geometry is not possible. But, having in mind the applications to deformation theory and especially the algebraization of formal moduli in the framework of analytic geometry (see $\left.\left[\mathrm{Bi}_{2}\right]\right)$, it is indeed possible to formulate an analogous assertion.

The methods of Palamodov, developed in $\left[\mathrm{Pa}_{1}, \mathrm{~Pa}_{2}\right]$ for the solution of the local moduli problem for compact complex spaces, and the expansion of these

Received by the editors September 12, 1989.

1980 Mathematics Subject Classification (1985 Revision). Primary 32G13, 14D15. 
techniques given in [B-K], can be used now to establish a proof for this analytic version. The idea has been known to the author for a long time, but enormous technical problems had to be overcome and this was achieved quite recently. One crucial notion is that of a special resolvent and a special resolution (see $\S \S 5$ and 6), which is a refined version of appropriate data from [B-K] ("zulässige Resolventen"). It should be mentioned that this paper relies heavily on the book [B-K] and only some indispensable definitions are repeated in the appendix.

We are even able to show an existence theorem for formal spaces, by using the same approach (Theorem (1.7)), and for which no algebraic analogue seems to be known. As one expects, local existence theorems hold too. But here the proof is easy in comparison to the global case.

Some applications of the main results are discussed in $\S 12$. Among others, we can prove the following assertion in full generality: Every 1-convex space $X$ with exceptional set $E$ and $\operatorname{supp}\left(\mathscr{T}^{1}\left(X, \mathscr{O}_{X}\right)\right) \subset E$ has a semiuniversal deformation as a germ along $E$. The essential step for this is to show a formal principle for deformations of 1-convex spaces (the rest is done analogously like in [B-K, Kap. $\mathrm{V}, \S 5]$ ), which is a consequence of the existence theorem for formal sheaves and Artin's approximation theorem.

Our main theorem, Theorem (1.2), seems to be very useful for the representation of the Chow functor in analytic geometry. But since this functor has not yet been explicitly defined in the analytic case, we did not touch this question here.

The main part of this work has been obtained at the Sonderforschungsbereich 170 Geometrie und Analysis in Göttingen to whom the author wants to express his sincere thanks. Moreover, I want to mention that several statements in this paper were previously known to J. Bingener, as for example the relative quotient theorem $(\S 3)$ and the deduction of the formal principle for 1-convex maps from the analytic existence theorem for sheaves. He also informed me that P. Deligne asked if an existence theorem for formal spaces (like in (1.7)) holds too.

\section{Formulation of the MAIN Results}

(1.1) We call a C-algebra $A$ formal, if it is a quotient of an algebra of the form $(K)^{\wedge \mathfrak{a}}$, where $K=\mathbf{C}\left\{x_{1}, \ldots, x_{m}\right\}$ is a convergent power series ring and " $\wedge \mathrm{a}$ " means completion with respect to the ideal $\mathfrak{a} \subset \mathfrak{m}_{K}$. So we have $A=(K)^{\wedge \mathfrak{a}} / \mathfrak{b}$ with an ideal $\mathfrak{b}$ in $(K)^{\wedge a}$, contained in the maximal ideal. Especially, every analytic C-algebra is a formal one. We associate to $A$ a space germ $S=(0, A)$, consisting set-theoretically of just one point which we always denote by 0 .

The category $\operatorname{An}(A)$ - or $\operatorname{An}(S)$ - of complex spaces over $A$ is defined in a natural way. The local models in $\operatorname{An}(A)$ are given as follows: The smooth spaces $M$ over $A$ consist (locally) of an open subset $D$ in some $C^{n}$ with the structure sheaf $\mathscr{O}_{M}=\mathscr{O}_{D}\left\{x_{1}, \ldots, x_{m}\right\}^{\wedge \mathfrak{a}} / \mathfrak{b} \mathscr{O}_{D}\left\{x_{1}, \ldots, x_{m}\right\}^{\wedge a}$, or, more invariantly, $\mathscr{O}_{M}=\lim _{k} \mathscr{O}_{D} \hat{\otimes}_{\mathbf{C}}\left(A / \overline{\mathfrak{a}}^{k+1}\right)$, where $\overline{\mathfrak{a}}$ is the image of $\mathfrak{a}$ in $A$ (which we denote from now on also by $\mathfrak{a}$ ). The general local pieces are of 
the form $X=\left(V(\mathscr{I}),\left.\left(\mathscr{O}_{M} / \mathscr{I}\right)\right|_{V(\mathscr{F})}\right)$, where $\mathscr{I} \subset \mathscr{O}_{M}$ is a coherent ideal and $V(\mathscr{I}) \subset D$ is the zero-set of $\mathscr{I}$ (observe that $\mathscr{O}_{M}$ is a coherent sheaf of rings). Obviously, one has products in $\operatorname{An}(A)$ and also a base change functor $\operatorname{An}(A) \rightarrow \operatorname{An}(B), X \mapsto X_{B}$, for each continuous morphism $A \rightarrow B$ of formal algebras. For any space $X$ in $\operatorname{An}(A)$, the spaces $X_{A / a^{k+1}}$ are so-called pseudocomplex spaces, i.e., C-ringed spaces $Y$ which are locally of the form $\mathscr{O}_{Z} \| Y \mid$, where $Z$ is a complex space and $|Y| \subset Z$ a locally closed subspace (in our case this subset is even analytic). In the case where $A$ is $\mathfrak{m}_{A}$-adically complete, the category $\operatorname{An}(A)$ is just the category of those formal complex spaces $X$ over $A$, for which $\mathfrak{m}_{A} \mathscr{O}_{X}$ is an ideal of definition for $X$.

Let $S=(S, 0)$ be a usual complex space germ, $f: X \rightarrow S$ a separated holomorphic map, and $A$ a formal algebra over $\Lambda:=\mathscr{O}_{S, 0}$. In the following, we consider exhaustions of $A$,

$$
A=\underset{i \in I}{\lim } A_{i}
$$

by analytic $\Lambda$-subalgebras $A_{i}$ of $A$ with filtered index set $I$. Such exhaustions always exist. For a formal space germ $T$ over $S$, we put $X_{T}:=T \times_{S} X$ and $f_{T}: X_{T} \rightarrow T$ denotes the base change of $f$ with respect to $T \rightarrow S$. If $B$ is the algebra belonging to $T$, we also write $X_{B}$ and $f_{B}$ instead of $X_{T}$ and $f_{T}$. Let $Y$ be a space in $\operatorname{An}(A)$, then we denote by $\operatorname{Coh}_{p}(Y), \operatorname{resp} . \operatorname{Coh}_{p, f}(Y)$, the full subcategories of the category $\operatorname{Coh}(Y)$ of coherent sheaves on $Y$ which consists of those modules having compact support, resp. those which are in addition flat over $A$. We now get the following functors by base change:

$$
\begin{aligned}
\underset{i \in I}{\lim _{i \in}} \operatorname{Coh}_{p}\left(X_{A_{i}}\right) & \rightarrow \operatorname{Coh}_{p}\left(X_{A}\right), \\
\underset{i \in I}{\lim } \operatorname{Coh}_{p, f}\left(X_{A_{i}}\right) & \rightarrow \operatorname{Coh}_{p, f}\left(X_{A}\right) .
\end{aligned}
$$

Note that each $A_{i}$ is here considered as a formal algebra. By using known results, it is not difficult to see that (1.1.3) gives an equivalence of categories (compare $\left[\mathrm{Bi}_{2}\right.$, Satz (11.3)]). Also the functor

$$
\operatorname{Coh}\left(X_{A}\right) \rightarrow \underset{k}{\lim } \operatorname{Coh}\left(X_{A / a^{k+1}}\right)
$$

is an equivalence $\left(\right.$ see $\left.\left[\mathrm{Bi}_{1},(1.2),(1.14)\right]\right)$ and the same is true for $\operatorname{Coh}_{p}(\cdots)$ and $\operatorname{Coh}_{p, f}(\cdots)$. The surjectivity of (1.1.3) can be deduced from the theorem of Siu and Trautmann [S-T] concerning the existence of semiuniversal deformations of coherent sheaves with compact supports, whilst the fully faithfulness is a consequence of a theorem of Pourcin [Pou]. Vice versa, it is possible to derive the result of Siu and Trautmann from the equivalence (1.1.3) by using only abstract deformation theory. One main theorem of this paper is the following assertion.

(1.2) Theorem. The functor (1.1.2) gives an equivalence of categories. 
(1.3) Corollary. If $X \rightarrow S$ is proper and $\operatorname{Coh}_{p}(\cdots)$ is replaced by the category $\operatorname{Vect}(\cdots)$ of holomorphic vector bundles, then

$$
\underset{i \in I}{\lim _{i \in I}} \operatorname{Vect}\left(X_{A_{i}}\right) \rightarrow \operatorname{Vect}\left(X_{A}\right)
$$

is an equivalence of categories.

(1.4) Next we are going to formulate an analogous assertion to (1.2) for the case of spaces. We fix an exhaustion like in (1.1.1) with $\Lambda=\mathbf{C}$. For any formal algebra $B$, we denote by $\operatorname{An}_{p}(B)$, resp. $\mathbf{A n}_{p, f}(B)$, the full subcategory of $\operatorname{An}(B)$, consisting of those spaces whose underlying topological space is compact, resp. those which are in addition flat over $B$. Again, by base change, we obtain functors

$$
\begin{aligned}
\underset{i \in I}{\lim } \mathbf{A} \mathbf{n}_{p}\left(A_{i}\right) & \rightarrow \mathbf{A} \mathbf{n}_{p}(A), \\
\underset{i \in I}{\lim } \mathbf{A} \mathbf{n}_{p, f}\left(A_{i}\right) & \rightarrow \mathbf{A n} \mathbf{n}_{p, f}(A) .
\end{aligned}
$$

Standard results in deformation theory imply

(1.5) Proposition. The functor (1.4.2) is an equivalence of categories.

Proof. Surjectivity. Let $T$ be the space germ associated to $A$ and $\mathscr{X} \rightarrow T$ an object in $\operatorname{An}_{p, f}(A)$. Then the special fiber $X_{0}$ in $0 \in T$ is a compact complex space. Now, denote by $Y \rightarrow S$ the semiuniversal deformation of $X_{0}$. By versality, we get a cartesian diagram

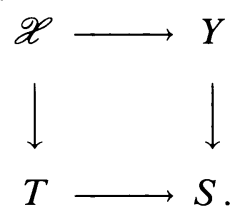

Since the morphism $\mathscr{O}_{S, 0} \rightarrow A$ necessarily factorizes over some $A_{i}$, we get $\left(Y_{A_{i}}\right)_{A} \cong \mathscr{X}$, so we are done.

Fully faithfulness. Let $X$ and $Y$ be spaces in $\operatorname{An}_{p, f}\left(A_{i}\right)$ and $f: X_{A} \rightarrow Y_{A}$ a morphism in $\operatorname{An}_{p, f}(A)$. Then the complex space $H:=\operatorname{Hom}_{A_{i}}(X, Y)$, defined over $A_{i}$, has a section after base change with $A_{i} \rightarrow A$. The distinguished point of $H$ is given here by $f(0): X_{A}(0) \rightarrow Y_{A}(0)$. We have a cornmutative diagram

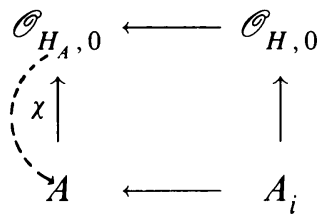

with a section $\chi$. The restriction of $\chi$ to $\mathscr{O}_{H, 0}$ factorizes over some $A_{j}$ with $j \geq i$, and therefore we get a section of $A_{j} \rightarrow \mathscr{O}_{H_{A_{j}}, 0}$. This shows the surjectivity of

$$
\underset{j \geq i}{\lim _{j}} \operatorname{Hom}_{A_{j}}\left(X_{A_{j}}, Y_{A_{j}}\right) \rightarrow \operatorname{Hom}_{A}\left(X_{A}, Y_{A}\right) .
$$


Now, let $f, g: X_{A_{i}} \rightarrow Y_{A_{i}}$ be two morphisms with $f_{A}=g_{A}$. Then $f$ and $g$ induce two sections of $A_{i} \rightarrow \mathscr{O}_{H, 0}$, which are equal after base change with $A$. But $A_{i} \rightarrow A$ is injective, and so $f=g$. This shows the proposition.

(1.6) Remark. Assuming the conclusion in (1.5), one can derive the existence of convergent formally semiuniversal deformations of compact complex spaces by general deformation theory (compare $\left[\mathrm{Bi}_{2}, \operatorname{Korollar}(2.7)\right]$ ).

The second main result is now

(1.7) Theorem. The functor (1.4.1) gives an equivalence of categories.

This assertion can be considered as an existence theorem for formal spaces. The proof of (1.2) and (1.7) uses (for the local description of spaces and sheaves) the theory of resolvents, as developed in [B-K, (I.8)]. For our purpose very special resolvents are necessary, even more special ones than those considered in [B-K, Kap. V]. This is rather essential for the proof. Furthermore, we need a relative version of the quotient theorem in [loc. cit., (II.12)], which is given in $\S 3$. Concerning some other applications not considered in this paper, it is useful to state it in a very general way.

(1.8) We are now going to treat the "local" case which is somewhat different. Let $(X, 0) \rightarrow(S, 0)$ be a morphism of complex space germs. For a formal algebra $B$ over $\mathscr{O}_{S, 0}$, we denote by $\operatorname{Coh}^{\prime}\left(X_{B}, 0\right)$ the full subcategory of $\operatorname{Coh}\left(X_{B}, 0\right)$ consisting of those germs of modules whose singularity set is finite over $B$. Fix now a formal $\mathscr{O}_{S, 0}$-algebra $A$ together with an exhaustion $\left(A_{i}\right)_{i \in I}$ as in (1.1.1). Then we have

(1.9) Theorem. Let $(X, 0) \rightarrow(S, 0)$ be flat. Then the functors

$$
\begin{aligned}
& \underset{i \in I}{\lim _{i}} \operatorname{Coh}^{\prime}\left(X_{A_{i}}, 0\right) \rightarrow \operatorname{Coh}^{\prime}\left(X_{A}, 0\right), \\
& \operatorname{Coh}^{\prime}\left(X_{A}, 0\right) \rightarrow \underset{k}{\lim } \operatorname{Coh}^{\prime}\left(X_{A / a^{k+1}}, 0\right)
\end{aligned}
$$

are surjective. Here $\mathfrak{a} \subset A$ is an ideal of definition.

The functors in (1.9) are in general not equivalences.

Next we consider the case of spaces. Let $A$ be a formal $\mathbf{C}$-algebra. Then we denote by $\operatorname{Gan}(A)$ the category of complex space germs over $A$ and by $\operatorname{Gan}^{\prime}(A)$ the full subcategory whose objects $X$ have the property that the nonsmoothness locus of $X \rightarrow A$ is finite over $A$. If we fix an exhaustion $\left(A_{i}\right)_{i \in I}$ of $A$ by convergent subalgebras, we have

(1.10) Theorem. The functor

$$
\underset{i \in I}{\lim _{i}} \operatorname{Gan}^{\prime}\left(A_{i}\right) \rightarrow \operatorname{Gan}^{\prime}(A)
$$

is surjective. 
Unfortunately, our method does not yet apply to show that also

$$
\operatorname{Gan}^{\prime}(A) \rightarrow \underset{k}{\lim } \operatorname{Gan}^{\prime}\left(A / \mathfrak{a}^{k+1}\right)
$$

is surjective. But this should be true nevertheless.

Both results (1.9) and (1.10) are proved by using privileged neighborhoods for certain complexes and by applying essentially the same idea as in the proof of (1.2) or (1.7).

At last we want to mention that there is certainly a common generalization of Theorems (1.2) and (1.7) in the context of graduate (formal) spaces. But since the foundation of such a theory has not yet been developed enough, we do not enter into this context.

\section{INTRODUCTORY NOTATIONS AND DEFINITIONS}

The main part of this section is concerned with the notion of a relative POspace and a relative PO-Lie group, where the parameter spaces are banachanalytic space germs. In the absolute case, these constructions have been discussed in detail in [B-K, Kap. II]. As the approach given here is sufficient for our purpose, we have not intended to develop such a relative theory which gives precisely the one of $[\mathrm{B}-\mathrm{K}]$ in the absolute context.

(2.1) Let $\mathscr{C}$ be a category, $S$ an object of $\mathscr{C}$, and $F: \mathscr{C}_{S} \rightarrow$ (sets) a contravariant functor. For a morphism $S^{\prime} \rightarrow S$, we denote by $F_{S^{\prime}}$ the composition $\mathscr{C}_{S^{\prime}} \rightarrow \mathscr{C}_{S} \stackrel{F}{\rightarrow}$ (sets). If for example $\mathscr{C}$ is the category (Ban) of banachanalytic space germs and $S=(S, 0)$ is an object of $\mathscr{C}$, we also write $F(0)$ instead of $F_{0}$ (induced by the inclusion $0 \hookrightarrow S$ ) and call $F(0)$ the fiber of $F$.

(2.2) Let $S$ be a banachanalytic space germ and $\varepsilon \in] 0,1$. Then the category $\operatorname{Con}^{\varepsilon}(S)$ of PO-spaces over $S$ is defined in the following way:

The objects of $\operatorname{Con}^{e}(S)$ are pairs $(S, E)$ where $E$ is a PO-space (compare the appendix). We usually write such an object in the form $\left(E_{S}, 0\right)$. If $\left(E_{S}, 0\right)$ and $\left(F_{S}, 0\right)$ are two objects in $\operatorname{Con}^{\varepsilon}(S)$, a morphism $u:\left(E_{S}, 0\right) \rightarrow\left(F_{S}, 0\right)$ will consist of the following data and conditions:

(1) A morphism $u_{0}:(E, 0) \rightarrow(F, 0)$ in the absolute category $\operatorname{Con}^{\varepsilon}$ (compare [B-K, (II.8)]); so $u_{0}$ is a convergent morphism between PO-spaces.

(2) $S$-morphisms of banachanalytic space germs

$$
u_{\lambda^{\prime}, \lambda}: S \times\left(\bar{E}_{\lambda}, 0\right) \rightarrow S \times\left(\bar{F}_{\lambda^{\prime}}, 0\right)
$$

for $\lambda^{\prime}, \lambda \in\left[1-\varepsilon, 1\left[\right.\right.$ with $\lambda^{\prime}<\lambda$, such that the equality

$$
\left(\operatorname{id}_{S} \times i_{\lambda_{4}, \lambda_{2}}^{F}\right) \cdot u_{\lambda_{2}, \lambda_{1}}=u_{\lambda_{4}, \lambda_{3}} \circ\left(\operatorname{id}_{S} \times i_{\lambda_{3}, \lambda_{1}}^{E}\right)
$$

holds for $\lambda_{4}<\lambda_{2}<\lambda_{1}$ and $\lambda_{4}<\lambda_{3}<\lambda_{1}$; here $i_{\lambda^{\prime}, \lambda}^{E}: \bar{E}_{\lambda} \rightarrow \bar{E}_{\lambda^{\prime}}$ and $i_{\lambda^{\prime}, \lambda}^{F}: \bar{F}_{\lambda} \rightarrow$ $\bar{F}_{\lambda^{\prime}}$ are the canonical mappings (see appendix).

(3) $u_{\lambda^{\prime}, \lambda}(0)=\left(\bar{u}_{0}\right)_{\lambda^{\prime}, \lambda}$ for $\lambda^{\prime}<\lambda$.

The composition of two such morphisms is defined obviously. If one replaces " $<$ " by " $\leq$ " in the above definition, then, in particular, there are morphisms 
$u_{\lambda}: S \times\left(\bar{E}_{\lambda}, 0\right) \rightarrow S \times\left(\bar{F}_{\lambda}, 0\right)$ for $\lambda \in\left[1-\varepsilon, 1\left[\right.\right.$, and supposes $u_{0}$ to be strictly convergent (in the sense of [B-K, (II.8.6(2))], then we shall also call $u$ strictly convergent. In the case $\varepsilon=1 / 2$, we just write $\operatorname{Con}(S)$ instead of $\operatorname{Con}^{1 / 2}(S)$.

(2.3) Now, we fix $t_{0} \in\left[1 / 2,1\left[, \lambda_{.} \in\left[t_{0}, 1\right]\right.\right.$. Let $S, E$, and $F$ be as above and $\sigma: S \rightarrow S \times\left(\bar{F}_{\lambda_{0}}, 0\right)$ a section of the canonical projection

$$
\operatorname{pr}_{2}: S \times\left(\bar{F}_{\lambda_{.}}, 0\right) \rightarrow S .
$$

Then every strictly convergent morphism $u:\left(E_{S}, 0\right) \rightarrow\left(F_{S}, 0\right)$ in $\operatorname{Con}(S)$ induces an inductive system $W=\left(W_{\lambda}\right)_{\left.\lambda \in] t_{0}, \lambda_{\text {. }}\right]}$ of banachanalytic space germs over $S$, by setting

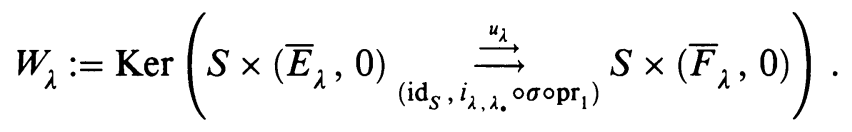

We call $W$ the fiber of $u$ in $\sigma$ and write $W=u^{-1}(\sigma)$. One can associate to $W$ a functor

$$
\begin{aligned}
& \check{W}_{t_{0}}: \text { Ban } / S \rightarrow(\text { sets }) \\
& T \mapsto \underset{\lambda>t_{0}}{\lim } \operatorname{Hom}_{S}\left(T, W_{\lambda}\right) .
\end{aligned}
$$

The fiber of this functor is, according to (2.1), denoted by $\check{W}_{t_{0}}(0)$. In the case $F=0$, we write $\left(\check{E}_{S}\right)_{t_{0}}$ for $\check{W}_{t_{0}}$, and we obviously have $\left(\check{E}_{S}\right)_{t_{0}}(0)=$ $\check{E}_{t_{0}}:$ Ban $\rightarrow$ (sets), $T \mapsto{\stackrel{\lim }{\longrightarrow} \lambda>t_{0}}_{\operatorname{Hom}}\left(T,\left(\bar{E}_{\lambda}, 0\right)\right)$.

(2.4) Let $G=\left(G_{\lambda}\right)_{\lambda \in] t_{0}, 1[}$ be an inductive system of banachanalytic space germs, contained in $\left(\bar{K}_{\lambda, S}, 0\right)_{\lambda}$ with a PO-space $K$. We say that $G$ is a POLie group over $S$, if there are $S$-morphisms of banachanalytic space germs (compatible in $\lambda^{\prime}, \lambda$ ):

$$
\begin{array}{ll}
m_{\lambda^{\prime}, \lambda}: G_{\lambda^{\prime}} \times{ }_{S} G_{\lambda} \rightarrow G_{\lambda^{\prime}} & \text { (multiplication), } \\
j_{\lambda^{\prime}, \lambda}: G_{\lambda} \rightarrow G_{\lambda^{\prime}} & \text { (inversion), } \\
e_{\lambda}: S \rightarrow G_{\lambda} & \text { (unit section) }
\end{array}
$$

for all $\lambda^{\prime}, \lambda$ with $t_{0}<\lambda^{\prime}<\lambda \leq \lambda$, such that the analogous properties for a group hold, like associativity, right-left-inverse, etc. (here whenever the composition of these morphisms $m_{\lambda^{\prime}, \lambda}, j_{\lambda^{\prime}, \lambda}$, etc. is defined). Observe that the functor $\check{G}_{t_{0}}$ is group valued.

If $G$ and $H$ are PO-Lie groups over $S$, then a homomorphism $u: G \rightarrow H$ is a morphism of the underlying inductive system which commutes with $m, j$, and $e$. The notions of a PO-Lie subgroup, of a normal PO-Lie subgroup, and of a semidirect product are defined in an obvious way. A PO-Lie group $G$ over $S$ is called strict if its "group-structure" is induced by germs of relative and compatible group structures on each $G_{\lambda}$. If $G$ is a strict PO-Lie group in 
the sense of [B-K, (II.9.2)], then $G_{S}$ has a natural structure of a strict relative PO-Lie group over $S$.

(2.5) Let $G$ be a PO-Lie group over $S$ and $W=\left(W_{\lambda}\right)_{\left.\lambda \in t_{0}, \lambda_{\text {. }}\right]}$ an inductive system of banachanalytic space germs over $S$. A (left)-operation of $G$ on $W$ of type $(-1,1 ;-1)$ is a compatible system of $S$-morphisms

$$
\omega_{\lambda^{\prime}, \lambda}: G_{\lambda^{\prime}} \times_{S} W_{\lambda} \rightarrow W_{\lambda^{\prime}}
$$

for $t_{0}<\lambda^{\prime}<\lambda \leq \lambda_{\text {. }}$, such that $\omega=\left(\omega_{\lambda^{\prime}, \lambda}\right)_{\lambda^{\prime}, \lambda}$ commutes with the multiplication on $G$ and $\omega_{\lambda^{\prime}, \lambda}\left(e_{\lambda^{\prime}} \times \mathrm{id}_{W_{\lambda}}\right)=i_{\lambda^{\prime}, \lambda}^{W}$. From this, we get an operation of $\check{G}_{t_{0}}$ on $\check{W}_{t_{0}}$ which is denoted by $\check{\omega}_{t_{0}}$. The operation $\omega$ is called strict if $\lambda^{\prime}=\lambda$ is also allowed. The notion of an equivariant morphism is obviously defined.

If now $\sigma: S \rightarrow W$ is an inductive system of sections of $W \rightarrow S$, then we call $\sigma$ fixed under the operation $\omega$ if each diagram

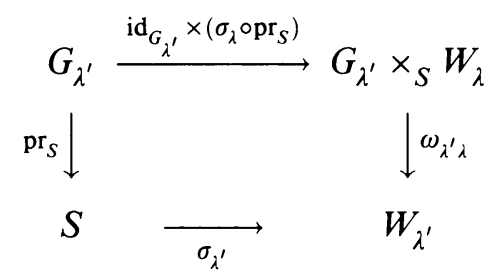

is commutative for $\lambda^{\prime}<\lambda$ (and for $\lambda^{\prime} \leq \lambda$ in the case of a strict operation).

Let $u: W \rightarrow V$ be an equivariant morphism (with respect to $G$ ) and $\sigma: S \rightarrow$ $V$ a section which is fixed under the operation. Then the operation of $G$ on $W$ induces one on $u^{-1}(\sigma)$.

(2.6) If $\varphi: S^{\prime} \rightarrow S$ is a morphism of banachanalytic space germs, we get a functor $\operatorname{Con}^{\varepsilon}(S) \rightarrow \operatorname{Con}^{\varepsilon}\left(S^{\prime}\right)$ via base change with $\varphi$. For $W$ in (2.3), we have $\left(\check{W}_{t_{0}}\right)_{S^{\prime}}=\left(W_{S^{\prime}}\right)_{t_{0}}^{v}$, where $W_{S^{\prime}}=\left(S^{\prime} \times_{S} W_{\lambda}\right)_{\lambda}$. The notions introduced in (2.4), (2.5) are consistent with base change. For example, if $G$ is a PO-Lie group over $S$, then the base changed object $G_{S^{\prime}}$ is again a PO-Lie group over $S^{\prime}$.

(2.7) Let $\omega: G \times{ }_{S} W \rightarrow W$ be an operation as in (2.5) and let us assume that $W$ is given as a subspace of $\left(E_{S}, 0\right)$ like in $(2.3)$ and $G$ embedded in $\left(K_{S}, 0\right)$ with a PO-space $K$. If $\sigma: S \rightarrow W$ is a section of $W \rightarrow S$, the orbit mapping $\beta: G \rightarrow W$ of $\omega$ in $\sigma$ is given by $\beta_{\lambda^{\prime}}=\omega_{\lambda^{\prime}, \lambda}\left(\mathrm{id}_{G_{\lambda^{\prime}}}, \sigma_{\lambda}\right)$ for $\lambda^{\prime}<\lambda$ and $\beta=\left(\beta_{\lambda^{\prime}}\right)_{\left.\lambda^{\prime} \in\right] t_{0}, \lambda_{\cdot}[}$.

Suppose now $S=\operatorname{Spec}(\mathbf{C})$. Then $\beta$ is called direct (with respect to $1-t_{0}$ ), if $\operatorname{Ker}\left(T\left(\beta_{\lambda}\right)\right)$ and $\operatorname{Im}\left(T\left(\beta_{\lambda}\right)\right)$ are complemented linear subspaces of the Banach space $\bar{K}_{\lambda}$, resp. $\bar{E}_{\lambda}$, and one has a compatible system of projections on them (here $\lambda$ varies in $] t_{0}, \lambda .[$ ).

(2.8) We are going to introduce briefly some notation concerning truncations of complexes. For this, let $\mathscr{C}$ be an additive category in which kernels exist. We fix a complex $K$ in $\mathscr{C}$ and a number $r$ in $\mathbf{Z} \cup\{ \pm \infty\}$. By $K^{[\leq r]}$ we denote the subcomplex of $K$ with $\left(K^{[\leq r]}\right)^{\nu}:=K^{\nu}$ for $\nu<r,\left(K^{[\leq r]}\right)^{\nu}:=\operatorname{Ker}\left(K^{\nu} \stackrel{d}{\rightarrow} K^{\nu+1}\right)$ 
for $\nu=r$ and $\left(K^{[\leq r]}\right)^{\nu}:=0$ for $\nu>r$. If we assume that cokernels also exist in $\mathscr{C}$, then the quotient complex $K^{[\geq r]}$ of $K$ is defined by $\left(K^{[\geq r]}\right)^{\nu}:=0$ for $\nu<r,\left(K^{[\geq r]}\right)^{\nu}:=\operatorname{Coker}\left(K^{\nu-1} \stackrel{d}{\rightarrow} K^{\nu}\right)$ for $\nu=r$ and $\left(K^{[\geq r]}\right)^{\nu}:=K^{\nu}$ for $\nu>r$.

Now, if $m, r$ are in $\mathbf{Z} \cup\{ \pm \infty\}$ with $m<r$, we combine the constructions above and get a complex $K^{[m, r]}$.

For $m=r$, we put $K^{[m, m]}:=H^{m}(K)[-m]$. Here $[k]$ means the shift to the $(-k)$ th place.

The usual foolish truncation of $K$ between $m$ and $r$ is denoted by $K^{(m, r)}$ (and also analogously by $K^{(\geq m)}, K^{(\leq r)}$ within the interval $[m,+\infty[$, resp. ] $-\infty, r])$. Here of course $\mathscr{C}$ may be arbitrary again.

\section{A RELATIVE QUOTIENT THEOREM}

This section is devoted to a relative and expanded version of the quotient theorem in [B-K, Kap. II, §12]. We begin with some general remarks.

(3.1) Let $F: \mathscr{C} \rightarrow$ (sets) and $G: \mathscr{C} \rightarrow$ (groups) be contravariant functors on a category $\mathscr{C}$ and suppose that an operation of $G$ on $F$ (from the left) is given. Then the quotient groupoid $F / G \stackrel{p}{\rightarrow} \mathscr{C}$ is defined as follows: The objects of $F / G$ over $T \in \mathscr{C}$ are the elements of $F(T)$. If $a^{\prime} \in F\left(T^{\prime}\right)$ and $a \in F(T)$ are two objects, then $\operatorname{Hom}_{F / G}\left(a^{\prime}, a\right)$ consists of all pairs $(f, g)$ in $\operatorname{Hom}_{\mathscr{E}}\left(T^{\prime}, T\right) \times G\left(T^{\prime}\right)$ with $g .(F(f)(a))=a^{\prime}$. Obviously, the set of isomorphy classes $\overline{(F / G)}(T)$ is equal to the orbit space $F(T) / G(T)$. Moreover, one has a functor $F / G \rightarrow \overline{F / G}$ of groupoids over $\mathscr{C}$. This is an equivalence of categories in the case when $G$ operates freely on $F$.

Now let $\mathscr{C}=\operatorname{Gan} / S$ be the category of complex space germs over a fixed germ $S$. We assume that $F$ and $G$ satisfy the Schlessinger condition $\left(S 1^{\prime}\right)$ from $\left[\mathrm{Bi}_{3}\right]$ and that $G$ is unobstructed (i.e., $G\left(T^{\prime}\right) \rightarrow G(T)$ is surjective for any $S$-embedding $\left.T \hookrightarrow T^{\prime}\right)$. One easily checks that also $F / G$ fulfills $\left(S 1^{\prime}\right)$. We fix a space $T$ in $\mathscr{C}$, a coherent $\mathscr{O}_{T}$-module $\mathscr{M}$ and an element $a \in F(T)$. Then the mapping $G_{1}(T[\mathscr{M}]) \rightarrow F_{a}(T[\mathscr{M}]),{ }^{1} g \mapsto g .\left(a_{T[\mathscr{M}]}\right)$ is a homomorphism of $\mathscr{O}_{T, 0}$-modules and the canonical surjection of $F_{a}(T[\mathscr{M}])$ onto $\overline{(F / G)_{a}}(T[\mathscr{M}])$ induces a functorial isomorphism

$$
F_{a}(T[\mathscr{M}]) / G_{1}(T[\mathscr{M}]) \stackrel{\sim}{\rightarrow}{\overline{(F / G)_{a}}}_{(}(T[\mathscr{M}])
$$

of $\mathscr{O}_{T, 0}$-modules.

(3.2) We fix a number $t_{0} \in[1 / 2,1[$. Let $S$ be a complex space germ, $G \rightarrow S$ a PO-Lie group over $S$ (see (2.4)) and $E, F$ two PO-spaces with an operation on $\left(E_{S}, 0\right)$ and $\left(F_{S}, 0\right)$. Moreover, we take a section $\sigma: S \rightarrow$ $\left(F_{S}, 0\right)$ of $\left(F_{S}, 0\right) \rightarrow S$, fixed by the operation of $G$, and a strictly convergent equivariant morphism $u:\left(E_{S}, 0\right) \rightarrow\left(F_{S}, 0\right)$ in $\operatorname{Con}(S)$. The operation of $G$

\footnotetext{
${ }^{1}$ Here $T[\mathscr{M}]$ is the trivial infinitesimal extension of $T$ by $\mathscr{M}$ and $G_{1}$, resp. $F_{a}$, is the fiber of $G$, resp. $F$, in $1_{G(T)}$, resp. $a$.
} 
on the fiber $W:=u^{-1}(\sigma)$ of $u$ in $\sigma$ induces an operation of $\check{G}_{t_{0}}$ on $\check{W}_{t_{0}}$. If in addition $G \rightarrow S$ is smooth, i.e., each morphism $G_{\lambda} \rightarrow S$ is smooth for $\lambda \in] t_{0}, 1\left[\right.$, then the quotient groupoid $\check{W}_{t_{0}} / \check{G}_{t_{0}}$, restricted to $(\mathbf{G a n} / S)$, fulfills the Schlessinger condition $\left(S 1^{\prime}\right)$. The following theorem tells us when $\check{W}_{t_{0}} / \check{G}_{t_{0}}$ has a semiuniversal deformation or is even representable.

(3.3) Theorem. The assumptions and notions are as in (3.2), and, moreover, we assume that the following conditions are satisfied:

(1) The operations $G \times_{S}\left(E_{S}, 0\right) \stackrel{\omega}{\rightarrow}\left(E_{S}, 0\right)$ and $G \times_{S}\left(F_{S}, 0\right) \rightarrow\left(F_{S}, 0\right)$ are of type $(-1,1 ;-1)$ and the spaces $E, F$ are $\left(1-t_{0}\right)$-good (see the appendix).

(2) The fiber operation of $G(0)$ on $(E, 0)$ is direct with respect to $\left(1-t_{0}\right)$-see (2.7)

(3) $u_{0}:(E, 0) \rightarrow(F, 0)$ is a direct morphism with respect to $\left(1-t_{0}\right)$, i.e., the tangent mapping $T\left(u_{0}\right): E \rightarrow F$ is direct in the category $\mathrm{PO}_{1-t_{0}}$.

(4) $\operatorname{dim}_{\mathbf{C}}\left(\left(\check{W}_{t_{0}}(0) / \check{G}_{t_{0}}(0)\right)\left(\operatorname{Spec}\left(\mathbf{C}[t] / t^{2}\right)\right)\right)<\infty$.

Then $\check{W}_{t_{0}} / \check{G}_{t_{0}}$ has a finite-dimensional semiuniversal deformation over $S$. If in addition

(5) the tangent mapping of the orbit map $G_{\lambda}(0) \rightarrow \bar{E}_{\lambda}$ is injective for all $\lambda \in$ ]$t_{0}, 1\left[\right.$, then the functor $\overline{\check{W}_{t_{0}} / \check{G}_{t_{0}}}$ of isomorphy classes on Ban/S is representable by a finite-dimensional space germ.

If (1)-(5) hold and the dimension in (4) is equal to zero, then $\overline{\check{W}_{t_{0}} / \check{G}_{t_{0}}}$ is representable by a closed subgerm of $S$.

(3.4) Remark. The dimension of the vector space in (3.3)(4) is zero, if $\check{G}_{t_{0}}$ operates freely and transitively on $\check{W}_{t_{0}}$, i.e., $\check{W}_{t_{0}}$ is a formal principal homogeneous "space" under the action of $\check{G}_{t_{0}}$.

Proof of the theorem. By (3) the image $I$ of $T\left(u_{0}\right): E \rightarrow F$ is a complemented summand of $F$ in $\mathrm{PO}_{1-t_{0}}$, so we have a retraction $v: F \rightarrow I$ onto $I$ in $\mathrm{PO}_{1-t_{0}}$. Let $W_{\lambda}$, resp. $M_{\lambda}$, be the inverse image of $\sigma_{\lambda}$, resp. $\left(\mathrm{id}_{s} \times \bar{v}_{\lambda}\right) \sigma_{\lambda}$, under the $S$-morphism $u_{\lambda}:\left(\bar{E}_{\lambda, S}, 0\right) \rightarrow\left(\bar{F}_{\lambda, S}, 0\right)$, resp. $\bar{v}_{\lambda, S} u_{\lambda}:\left(\bar{E}_{\lambda, S}, 0\right) \rightarrow$ $\left(\bar{I}_{\lambda, S}, 0\right)$, for $\left.\lambda \in\right] t_{0}, 1\left[\right.$. Then $W=\left(W_{\lambda}\right)_{\lambda \in] t_{0}, 1[}$ and $M=\left(M_{\lambda}\right)_{\lambda \in] t_{0}, 1[}$ are inductive systems of banachanalytic space germs over $S$ and each $M_{\lambda} \rightarrow S$ is smooth (see [Dou 2 , Proposition 2, p. 573]). Moreover, we have $W \subset M$. If $\beta: G \rightarrow\left(E_{S}, 0\right)$ denotes the orbit of the zero section $o$ of $\left(E_{S}, 0\right) \rightarrow S$ under the operation $\omega$, we get

$$
\left(\check{W}_{t_{0}}(0) / \check{G}_{t_{0}}(0)\right)(D)=\underset{\lambda>t_{0}}{\lim } T\left(W_{\lambda}(0)\right) / \operatorname{Im}\left(T\left(\beta_{\lambda}(0)\right)\right),
$$

with $D:=\operatorname{Spec}\left(\mathbf{C}[t] / t^{2}\right)$. Since, according to (1), $E$ is $\left(1-t_{0}\right)$-good, it follows from (2) that the transition homomorphisms from $T\left(W_{\lambda}(0)\right) / \operatorname{Im}\left(T\left(\beta_{\lambda}(0)\right)\right)$ to $T\left(W_{\lambda^{\prime}}(0)\right) / \operatorname{Im}\left(T\left(\beta_{\lambda^{\prime}}(0)\right)\right)$ are injective for $t_{0}<\lambda^{\prime} \leq \lambda<1$. Because of (3.3.1) 
and (4), there is an index $\lambda>t_{0}$ for which the canonical map from $T\left(W_{\lambda}(0)\right)$ to $\left(\check{W}_{t_{0}}(0) / \breve{G}_{t_{0}}(0)\right)(D)$ is surjective. Now we fix a finite-dimensional subgerm $N \subset M_{\lambda}$, smooth over $S$, such that the map $T(N(0)) \rightarrow\left(\check{W}_{t_{0}}(0) / \check{G}_{t_{0}}(0)\right)(D)$ is bijective. For the intersection $Y:=N \cap W_{\lambda}$, we get $T(Y / S)=T(N / S)$. From [Dou ${ }_{1}, 7.5$, Proposition 7], it follows that $Y$ is finite dimensional.

Let $\lambda^{\prime}$ be an arbitrary index with $t_{0}<\lambda^{\prime}<\lambda$. Then we have the following important assertion.

(3.3.2) Proposition. The morphism $\omega_{\lambda^{\prime}, \lambda}: G_{\lambda^{\prime}} \times_{S} Y \rightarrow W_{\lambda^{\prime}}$ is smooth.

For the proof of this statement, we choose subgerms $H$ and $K$ of $G_{\lambda^{\prime}}$, both smooth over $S$, and a $S$-retraction $p: G_{\lambda^{\prime}} \rightarrow K$ with the following properties:

(a) $e_{\lambda^{\prime}}: S \rightarrow G_{\lambda}$ factorizes over $H$ and $K$,

(b) $H=p^{-1}\left(e_{\lambda^{\prime}}\right)$,

(c) $T\left(G_{\lambda^{\prime}}(0)\right)=T(H(0)) \amalg T(K(0))$ and $T(K(0))=\operatorname{Ker}\left(T\left(\beta_{\lambda^{\prime}}(0)\right)\right)$.

The existence of such subgerms is easily seen by using the smoothness of $G_{\lambda^{\prime}} \rightarrow S$ and (2). Furthermore, we fix an $S$-retraction $q:\left(\bar{E}_{\lambda^{\prime}, S}, 0\right) \rightarrow M_{\lambda^{\prime}}$ and put

$$
\psi:=\left(p \circ \operatorname{pr}_{1}, q \circ \omega_{\lambda^{\prime}, \lambda}\right): G_{\lambda^{\prime}} \times{ }_{S} N \rightarrow K \times{ }_{S} M_{\lambda^{\prime}} .
$$

Then we obviously have the inclusions

$$
G_{\lambda^{\prime}} \times_{S} Y \subset \psi^{-1}\left(K \times_{S} W_{\lambda^{\prime}}\right) \subset G_{\lambda^{\prime}} \times_{S} N .
$$

If we denote by $i$, resp. $j$, the canonical $S$-morphism from $\bar{E}_{\lambda, S}$ to $\bar{E}_{\lambda^{\prime}, S}$, resp. from $\bar{F}_{\lambda, S}$ to $\bar{F}_{\lambda^{\prime}, S}$, and by $k: N \rightarrow G_{\lambda^{\prime}} \times_{S} N$ the inclusion given by $e_{\lambda^{\prime}}$, we can calculate

$$
\begin{aligned}
(\psi k)^{-1}\left(K \times{ }_{S} W_{\lambda^{\prime}}\right) & =(q \circ(i \mid N))^{-1}\left(W_{\lambda^{\prime}}\right)=(i \mid N)^{-1}\left(W_{\lambda^{\prime}}\right) \\
& =\left(u_{\lambda^{\prime}} \circ(i \mid N)\right)^{-1}\left(\sigma_{\lambda^{\prime}}\right)=\left(j \circ\left(u_{\lambda} \mid N\right)\right)^{-1}\left(\sigma_{\lambda^{\prime}}\right),
\end{aligned}
$$

since $q \circ(i \mid N)=i \mid N$ and $j u_{\lambda}=u_{\lambda^{\prime}} i$. As now $j(0)$ is injective (according to (1)) and $S$ is finite dimensional, the subgerm $\left(j \circ\left(u_{\lambda} \mid N\right)\right)^{-1}\left(\sigma_{\lambda^{\prime}}\right)$ coincides with $\left(u_{\lambda} \mid N\right)^{-1}\left(\sigma_{\lambda}\right)=Y$ by [B-K, (II.12.4)]. From [loc. cit., (II.12.6)] we get

$$
\psi^{-1}\left(K \times{ }_{S} W_{\lambda^{\prime}}\right)=G_{\lambda^{\prime}} \times_{S} Y .
$$

For elements $x \in T(K(0)), y \in T(H(0))$, and $z \in T(N(0))$, one obviously has the identity $T(\psi(0))(x+y, z)=\left(x, T\left(\beta_{\lambda^{\prime}}(0)\right)(y)+i(0)(z)\right)$. Therefore $T(\psi(0))$ maps $T\left(G_{\lambda}(0)\right) \times 0$ isomorphically onto $T(K(0)) \times \operatorname{Im}\left(T\left(\beta_{\lambda^{\prime}}(0)\right)\right)$. Since, by construction of $N$, the map $i(0)$ induces an isomorphism of $T(N(0))$ onto $T\left(M_{\lambda^{\prime}}(0)\right) / \operatorname{Im}\left(T\left(\beta_{\lambda^{\prime}}(0)\right)\right)$, the tangent map $T(\psi(0))$ is bijective. This implies by the relative implicit function theorem that $\psi$ itself is an isomorphism. So, in particular, $\psi$ gives an isomorphism from $G_{\lambda^{\prime}}{ }_{S} Y$ onto $K \times_{S} W_{\lambda^{\prime}}$. The 
commutative diagram

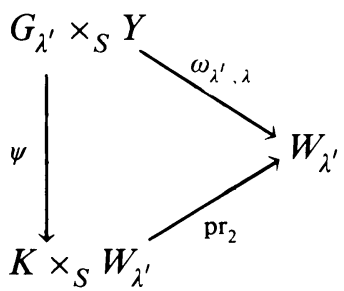

shows (3.3.2) and, moreover, also the first part of the assertion in (3.3) with $Y$ as a semiuniversal deformation.

Now, we prove the second assertion. According to the injectivity of the infinitesimal orbit maps $T\left(G_{\lambda^{\prime}}(0)\right) \rightarrow \bar{E}_{\lambda^{\prime}}$, it is possible to assume $H=G_{\lambda^{\prime}}$ in the proof above for $\left.\lambda^{\prime} \in\right] t_{0}, \lambda\left[\right.$. This implies that $\omega_{\lambda^{\prime}, \lambda}: G_{\lambda^{\prime}} \times_{S} Y \rightarrow W_{\lambda^{\prime}}$ is even an isomorphism and therefore the functor morphism

$$
\operatorname{Hom}_{S}(-, Y) \rightarrow \overline{\check{W}_{t_{0}} / \check{G}_{t_{0}}}
$$

is surjective, but also injective: Take $v, w \in \operatorname{Hom}_{S}(T, Y)$ with $T \in \operatorname{Ban} / S$, such that there is a $\left.\lambda^{\prime} \in\right] t_{0}, \lambda\left[\right.$ and $\alpha \in \operatorname{Hom}_{S}\left(T, G_{\lambda^{\prime}}\right)$ with $\omega_{\lambda^{\prime}, \lambda}(\alpha, v)=$ $i \circ w$, so $(\alpha, v)=\left(\omega_{\lambda^{\prime}, \lambda}\right)^{-1}(i \circ w)$. But since we have the commutative diagram

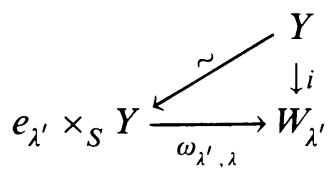

we get $\alpha=\left(e_{\lambda^{\prime}}\right)_{T}$ and this gives $i v=i w$. By [B-K, (II.12.4)], we obtain $v=w$.

The last assertion is true, because one can choose $N \stackrel{\sim}{\rightarrow} S$ in the proof above and so $Y \rightarrow S$ is an embedding. This completes the proof of (3.3).

At last we want to formulate a kind of finite-dimensional analogue of the construction in the proof of (3.3), which is often quite useful.

(3.5) Theorem (on relative transversal slices). Let $G \rightarrow S, X \rightarrow S$ be complex space germs over the germ $S$, where $G$ is in addition a relative Lie group germ over $S$. Moreover, let $\omega: G \times_{S} X \rightarrow X$ be an operation with orbit map $\beta_{0}: G(0) \rightarrow X(0)$ over the special fiber.

If $G \rightarrow S$ is smooth, then there exists a subgerm $Y \subset X$ (not necessarily unique) with the properties

(1) $\omega: G \times{ }_{S} Y \rightarrow X$ is smooth,

(2) $T(Y(0)) \cong T(X(0)) / \operatorname{Im}\left(T\left(\beta_{0}\right)\right)$.

If in addition $\check{G}=\operatorname{Hom}_{S}(-, G)$ operates freely on $\check{X}=\operatorname{Hom}_{S}(-, X)$, then $\omega: G \times{ }_{S} Y \rightarrow X$ is an isomorphism; if $X$ is a formal principal homogeneous space under the action of $\check{G}$, then $Y \rightarrow S$ is an embedding.

The proof of (3.5) is left to the reader as any easy exercise. 


\section{FORMAL MAPS INTO BANACHANALYTIC SPACE GERMS; FORMAL PO-MODULES}

(4.1) Let $T=(0, A)$ be a formal germ as in (1.1) and $\mathfrak{a} \subset A$ an ideal of definition. We write $A$ as a quotient $A=K^{\wedge \mathfrak{a}} / \mathfrak{b}$, where $K=\mathbf{C}\left\{x_{1}, \ldots, x_{m}\right\}$ is a smooth analytic algebra. For any $A$-module $M$, we put

$$
M_{k}:=M / \mathfrak{a}^{k+1} M, \quad k \in \mathbf{N},
$$

and $T_{k}$ denotes the germ $\left(0, A_{k}\right)$. If now $Z$ is a banachanalytic space or space germ, we define

$$
\operatorname{Hom}(T, Z):=\underset{k}{\lim } \operatorname{Hom}\left(T_{k}, Z\right) .
$$

Obviously, $\operatorname{Hom}(T, Z)$ does not depend on the choice of $\mathfrak{a}$.

Let $D^{\prime} \subset \subset D \subset \subset \mathbf{C}^{n}$ be open polydiscs and $X$ be the space $\left(\mathbf{C}^{n}\right)_{A}$ in $\operatorname{An}(A)$. Then we have

(4.2) Proposition. (i) There is a natural commutative diagram

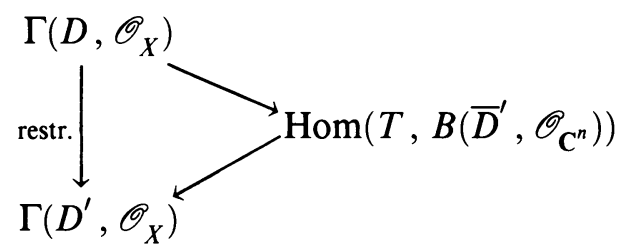

where $B\left(\bar{D}^{\prime}, \mathscr{O}_{\mathbf{C}^{n}}\right)$ is the Banach space of continuous maps $\bar{D}^{\prime} \rightarrow \mathbf{C}$ which are holomorphic on $D^{\prime}$.

(ii) If $D^{(\lambda)}$ denotes the shrinking of $D$ by the factor $\lambda$ with $\lambda \in[0,1[$, we have for $t_{0} \in[0,1[$ :

$$
\Gamma\left(\bar{D}^{\left(t_{0}\right)}, \mathscr{O}_{X}\right)=\underset{\lambda>t_{0}}{\lim } \operatorname{Hom}\left(T, B\left(\bar{D}^{(\lambda)}, \mathscr{O}_{\mathbf{C}^{n}}\right)\right) .
$$

(In the case $t_{0}=0$, the space $\bar{D}^{\left(t_{0}\right)}$ is just the origin of $D$. .)

Proof. The first part of the assertion is immediately reduced to the case $A=A_{k}$ where we can apply [Dou,$\S 5.2]$. The second part follows from the first one.

(4.3) Now, let $E$ be a complete PO-space. For a convergent C-algebra $A=$ $\mathbf{C}\{x\} / \mathfrak{b}$, we put

$$
E_{A}:=E\{x\} / \mathfrak{b} E\{x\} .
$$

Then $E_{A}$ is an $A$-module (which we call pseudofree), functorial in $A$ and independent of the chosen representation. More generally, if $A=\hat{K}^{\mathfrak{a}} / \mathfrak{b}$ is formal, we define

$$
E_{A}:=\underset{k}{\lim } E_{A_{k}} \cdot
$$

Then we have $E_{A}=E_{\hat{K}^{\mathrm{a}}} / \mathfrak{b} E_{\hat{K}^{\mathrm{a}}}$ and

$$
E_{A} \cong \underset{\lambda}{\lim }\left(\bar{E}_{\lambda}\right)_{A}
$$


We set

$$
E_{A}^{0}:=\operatorname{Ker}\left(E_{A} \rightarrow E_{A / \mathrm{m}_{A}}\right)
$$

Obviously, $E_{A}^{0}=\mathfrak{m}_{A} E_{A}$.

Let $A$ be formal and $Z$ a banachanalytic space germ, given as the zero-set of an analytic map $\varphi:(E, 0) \rightarrow(F, 0)$ between Banach space germs with Taylor expansion $\varphi=\sum_{k>0} \varphi_{k}$. Then $\varphi$ induces a unique map ("composition by $\varphi$ "),

$$
\Phi_{A}: E_{A}^{0} \rightarrow F_{A}^{0}
$$

which is functorial in $A$ (with respect to continuous C-algebra homomorphisms $\alpha: A \rightarrow B)$ and is, in the case $A=K=\mathbf{C}\left\{x_{1}, \ldots, x_{m}\right\}$, given by

$$
\begin{gathered}
\Phi_{K}(f):=\sum_{\substack{\nu \in \mathbf{N}^{m} \\
\Phi_{K}}} \Phi_{K_{\nu}}(f)_{\nu} x^{\nu}, \\
\Phi_{K}(f)_{l=1}^{\infty} \sum_{\substack{\nu_{1}+\cdots+\nu_{l}=\nu, \nu_{i} \in \mathbf{N}^{m}}} \tilde{\varphi}_{l}\left(f_{\nu_{1}}, \ldots, f_{\nu_{l}}\right),
\end{gathered}
$$

where $f=\sum_{\nu} f_{\nu} x^{\nu}$ is the expansion of $f$ (with $f_{\nu} \in E$ ) and $\tilde{\varphi}_{l}: E \times \cdots \times E \rightarrow$ $F$ denotes the symmetric $l$-multilinear map associated to $\varphi_{l}$.

(4.4) Proposition. In the situation above, one has a functorial isomorphism

$$
\operatorname{Hom}(T, Z) \cong \Phi_{A}^{-1}(0) \text {. }
$$

Proof (sketch). Since $\Phi_{A}=\underline{\lim }_{k} \Phi_{A_{k}}$, we are reduced to the case where $A$ is analytic. The next reductions are that it is sufficient to assume $Z=(E, 0)$ and $A$ smooth, so $A=\mathbf{C}\left\{x_{1}, \ldots, x_{m}\right\}$. But here the assertion is trivial.

(4.5) Let $A=K / \mathfrak{b}$ be an analytic C-algebra with $K=\mathbf{C}\left\{x_{1}, \ldots, x_{m}\right\}$ smooth. On $K$ we have pseudonorms $\|\cdot\|_{\rho}, \rho \in \mathbf{R}_{>0}^{m}$, defined by $\left\|\sum_{\nu} a_{\nu} x^{\nu}\right\|_{\rho}$ $:=\sum_{\nu}\left|a_{\nu}\right| \rho^{\nu}$, which induce quotient pseudonorms on $A$, also denoted by $\|\cdot\|_{\rho}$. Moreover, there is a triangle set (in the sense of [B-K, (II.3.1)]) $\Delta \subset \mathbf{R}_{>0}^{m}$, a constant $C>0$ and a C-linear section $\tau: A \rightarrow K$ of $K \rightarrow A$, such that $\|\tau(a)\|_{\rho} \leq C\|a\|_{\rho}$ for all $a \in A$ and $\rho \in \Delta$. We call $\Delta$ privileged for $A$.

Let $E$ be a complete PO-space. By setting $\left\|\sum_{\nu} e_{\nu} x^{\nu}\right\|_{\rho, \lambda}:=\sum_{\nu}\left\|e_{\nu}\right\|_{\lambda} \rho^{\nu}$, we obtain p̈seudonorms $\|\cdot\|_{\rho, \lambda}$ on $E_{K}$ and quotient pseudonorms on $E_{A}$ (denoted by the same symbol) for any $\left.\rho \in \mathbf{R}_{>0}^{m}, \lambda \in\right] 0,1[$. The map $\tau$ induces a $\mathbf{C}$ linear section $\tau^{E}: E_{A} \rightarrow E_{K}$ of $E_{K} \rightarrow E_{A}$, such that $\left\|\tau^{E}(u)\right\|_{\rho, \lambda} \leq C\|u\|_{\rho, \lambda}$ for all $\rho \in \Delta$ and $\lambda \in] 0,1[$.

Let $F$ be a second complete PO-space and $\varepsilon \in] 0,1[$. An $A$-linear map $\varphi: E_{A} \rightarrow F_{A}$ is called a $\mathrm{PO}_{\varepsilon}(A)$-morphism if there are $C>0$ and $\rho_{0}=\rho_{0}(\varphi) \in$ $\mathbf{R}_{>0}^{m}, \rho_{0} \leq(1, \ldots, 1),{ }^{2}$ such that

$$
\|\varphi(u)\|_{\rho, \lambda} \leq C\|u\|_{\rho, \lambda}
$$

\footnotetext{
${ }^{2}$ This means " $\leq$ " in each coordinate.
} 
for $u \in E_{A}, \lambda \in\left[1-\varepsilon, 1\left[\right.\right.$, and $\rho \leq \rho_{0}$. The category $\mathrm{PO}_{\varepsilon}(A)$ consists of all $A$-modules of the form $E_{A}$, with $E$ a complete $\mathrm{PO}$-space, and of the $\mathrm{PO}_{\varepsilon}(A)$ morphisms just defined. We call $E_{A}$ nuclear free if $E$ is a nuclear PO-space (compare the appendix). Furthermore, we set

$$
\text { PO. }(A)=\underset{\varepsilon \in] 0,1[}{\lim } \mathrm{PO}_{\varepsilon}(A) \text {. }
$$

Observe that every $\mathrm{PO}_{\varepsilon}(A)$-morphism is induced by a $\mathrm{PO}_{\varepsilon}(K)$-morphism: Let $\varphi: E_{A} \rightarrow F_{A}$ be in $\mathrm{PO}_{\varepsilon}(A)$ and denote by $\psi$ the composition

$$
E \stackrel{\varphi \mid E}{\longrightarrow} F_{A} \stackrel{\tau^{F}}{\longrightarrow} F_{K} \text {. }
$$

Then $\|\psi(e)\|_{\rho, \lambda} \leq C^{\prime}\|e\|_{\lambda}$ for all $\lambda \in\left[1-\varepsilon, 1\left[\right.\right.$ and $\rho \in \Delta, \rho \leq \rho_{0}(\varphi)$. Since $\|\cdot\|_{\rho^{\prime}, \lambda} \leq\|\cdot\|_{\rho, \lambda}$ for $\rho^{\prime} \leq \rho$, this estimate even holds for all $\rho \leq \rho_{0}$ with a fixed $\rho_{0} \in \Delta, \rho_{0} \leq \rho_{0}(\varphi)$. Obviously, the canonical $K$-linear extension of $\psi$ to $E_{K}$ has the desired property.

(4.6) Examples. (1) Let $\varphi_{\nu}: E \rightarrow F, \nu \in \mathbf{N}^{m}$, be a sequence of $\mathrm{PO}_{\varepsilon^{-}}$ morphisms, such that there are constants $C, R>0$ with

$(*) \quad\left\|\varphi_{\nu}(e)\right\|_{\lambda} \leq C R^{|\nu|}\|e\|_{\lambda}, \quad \forall e \in E, \forall \nu \in \mathbf{N}^{m}, \forall \lambda \in[1-\varepsilon, 1[$.

Then the $\mathbf{C}\{x\}$-linear map $\varphi: E\{x\} \rightarrow F\{x\}$, given on $E \subset E\{x\}$ by $\varphi(e):=$ $\sum_{\nu \in \mathbf{N}^{m}} \varphi_{\nu}(e) x^{\nu}$, is a morphism in $\mathrm{PO}_{\varepsilon}(\mathbf{C}\{x\})$. Moreover, the estimate $(*)$ characterizes $\mathrm{PO}_{\varepsilon}(\mathbf{C}\{x\})$-morphisms.

(2) Let $D \subset \subset D^{\prime} \subset \subset \mathbf{C}^{n}$ be two open polydiscs, $p, q \in \mathbf{N}$, and $M$ a matrix in $M\left(p \times q, \Gamma\left(D^{\prime}, \mathscr{O}_{\mathbf{C}^{n}}\right)_{A}\right)$. We set $E:=\Gamma\left(D, \mathscr{O}_{\mathbf{C}^{n}}\right)$. Then $M$ induces a $\mathrm{PO}_{\varepsilon}(A)$-morphism $M: E_{A}^{q} \rightarrow E_{A}^{p}$ by multiplication with $M$ for each $\left.\varepsilon \in\right] 0,1[$.

(4.7) Let $A \rightarrow A^{\prime}$ be a morphism of analytic algebras, where $A^{\prime}$ is considered as a quotient of the smooth algebra $K^{\prime}=\mathbf{C}\left\{x_{1}^{\prime}, \ldots, x_{m^{\prime}}^{\prime}\right\}$. Then for any $\mathrm{PO}_{\varepsilon}(A)$-morphism $\varphi: E_{A} \rightarrow F_{A}$, the base change $\varphi_{A^{\prime}}: E_{A^{\prime}} \rightarrow F_{A^{\prime}}$ is defined in $\mathrm{PO}_{\varepsilon}\left(A^{\prime}\right)$, and $\mathrm{PO}_{\varepsilon}(*)$ is functorial in $*$.

(4.8) We want to generalize these notions above to formal algebras $A$, given as usual as a quotient $A=K^{\wedge \mathfrak{a}} / \mathfrak{b}$. The objects of the category $\mathrm{PO}_{\varepsilon}(A)$ are the $A$-modules of the form $E_{A}$, with $E$ a complete PO-space. The morphisms in $\mathrm{PO}_{\varepsilon}(A)$ are by definition projective limits $\varphi=\varliminf_{k} \varphi_{k}$ of $\mathrm{PO}_{\varepsilon}\left(A_{k}\right)$-morphisms $\varphi_{k}: E_{A_{k}} \rightarrow F_{A_{k}}$. Here each $A_{k}$ is considered as a quotient of $K$. Observe that $\rho_{0}\left(\varphi_{k}\right)$ may shrink to 0 for $k \rightarrow \infty$. The example (4.6)(2) holds in this context too. We set

$$
\text { PO. }(A):=\underset{\varepsilon \in] 0,1[}{\lim } \mathrm{PO}_{\varepsilon}(A)
$$

and call the objects of PO. $(A)$ formal PO-modules over $A$. Again, there is a base change functor $\mathrm{PO}_{\varepsilon}(A) \rightarrow \mathrm{PO}_{\varepsilon}\left(A^{\prime}\right)$ for $A \rightarrow A^{\prime}$ continuous.

(4.9) Remark. Let $A$ be a formal algebra and $\varphi: E_{A} \rightarrow E_{A}$ a morphism in $\mathrm{PO}_{\varepsilon}(A)$. Then $\varphi$ is invertible in $\operatorname{PO}_{\varepsilon}(A)$ iff $\varphi_{A / \mathrm{m}_{A}}: E \rightarrow E$ is invertible in $\mathrm{PO}_{\varepsilon}$. 
For the proof, one immediately reduces to the situation $A=\mathbf{C}\left\{x_{1}, \ldots, x_{m}\right\}$. In this case the assertion follows from standard estimates in the proof of the (analytic) local inversion theorem by using (4.6)(*).

\section{SPECIAL POLYHEDRON FAMILIES AND RESOLVENTS}

In this section we are going to cut a space $\mathscr{Z}$ in $\operatorname{An}(A)$ into simplicial pieces and then construct a very special kind of resolution of the structure sheaf which will be crucial for the proof of (1.7). The construction goes along the lines of [B-K, Kap. IV, $\S \S 1-3]$, and so we shall be brief at those points which are analogous to loc. cit.

(5.1) Let $X$ be a (usual) complex space. By a quasi-polyhedron for $X$, we understand a triple $(P, \varphi, E)$, consisting of a compact subset $P$ of $X$, a closed embedding $\varphi: U \rightarrow V$ of complex spaces, where $U$ is open in $X$ and $V$ open in some $\mathbf{C}^{n}$, and an open polydisc $E \subset \subset \mathbf{C}^{n}$ with center $x_{0}$, such that $\bar{E} \subset V$ and $P=\varphi^{-1}(\bar{E})$. We call $(P, \varphi, E)$ a polyhedron for $X$ if $\varphi^{-1}\left(x_{0}\right)$ is not empty; the point $x \in P$ with $\varphi(x)=x_{0}$ is considered as the center of $(P, \varphi, E)$. Moreover, we set $\dot{P}:=\varphi^{-1}(E)$.

The morphism $\varphi$ induces a closed embedding $P \rightarrow \bar{E}$ of pseudocomplex spaces which is also denoted by $\varphi$. In the following, we just write $P$ instead of $(P, \varphi, E)$ and $U\langle P\rangle, V\langle P\rangle, \varphi\langle P\rangle, E\langle P\rangle$ for $U, V, \ldots$.

(5.2) Now, let $T=(0, A)$ be a formal space germ, $\mathfrak{a} \subset A$ an ideal of definition, and $\mathscr{X}$ a space in $\operatorname{An}(A)$ with structure map $\mathfrak{f}: \mathscr{X} \rightarrow T$ which is assumed to be separated. We fix a representation $A=K^{\wedge \mathfrak{a}} / \mathfrak{b}$ as in (1.1) and denote by $Z$, resp. $\hat{Z}$, the smooth space germ $(0, K)$, resp. $\left(0, K^{\wedge a}\right)$. Then a quasi-polyhedron $(P, \varphi, E)$ for $\mathscr{X} / T$ will consist of a closed subset $P \subset \mathscr{X}$, a closed embedding $\varphi: U \rightarrow \hat{Z} \times V$ in $\operatorname{An}(\hat{Z})$, where $U \subset \mathscr{X}$ is open and $V$ is open in some $\mathbf{C}^{n}$, and an open polydisc $E \subset \subset \mathbf{C}^{n}$ with center $x_{0}$, such that $\bar{E} \subset V$ and $\varphi^{-1}(\{0\} \times \bar{E})=P$. If $\varphi^{-1}\left(\{0\} \times\left\{x_{0}\right\}\right)$ is not empty, we call $P=(P, \varphi, E)$ a polyhedron for $\mathscr{X} / T$. The further notions of (5.1) also transpose into this context.

For each $t \in] 0,1]$, the (concentric) shrinking $P^{(t)}=\left(P^{(t)}, \varphi, E^{(t)}\right)$ of $P$ by the factor $t$ is again a (quasi-)polyhedron for $\mathscr{Z} / T$.

(5.3) Let $\mathscr{P}=\left(P_{i}, \varphi_{i}, E_{i}\right)_{i \in I}$ be a family of polyhedrons for $\mathscr{X} / T$. The nerve $\operatorname{Ner}(\mathscr{P})$ of $\mathscr{P}$ is by definition the nerve of the family $\left(P_{i}\right)_{i \in I}$ of compact subsets of $\mathscr{X}$, considered as an abstract simplicial scheme. The subset $|\mathscr{P}|:=$ $\bigcup_{i \in I} P_{i}$ of $\mathscr{X}$ is called the support of $\mathscr{P}$. We usually assume $\mathscr{P}$ to be locally finite, i.e., the family $\left(U\left\langle P_{i}\right\rangle\right)_{i \in I}$ is locally finite. If $t=\left(t_{i}\right)_{i \in I}$ is a sequence of numbers in $] 0,1]$, the shrinking $\mathscr{P}^{(t)}=\left(P_{i}^{\left(t_{i}\right)}, \varphi_{i}, E_{i}^{\left(t_{i}\right)}\right)_{i \in I}$ is again a family of polyhedrons for $\mathscr{X} / T$. In the case where all $t_{i}=s$ are equal, we also write $\mathscr{P}^{(s)}$ for $\mathscr{P}^{(t)}$. Furthermore, we put

$$
n(\mathscr{P}):=\sup \left\{\operatorname{dim} Z+\operatorname{dim} E_{\nu}+\sum_{i \in I} \operatorname{dim} E_{i} ; \nu \in I\right\} .
$$


For $\alpha=\left\{i_{0}, \ldots, i_{r}\right\}$ in $\operatorname{Ner}(\mathscr{P})$, we define $E_{\alpha}:=E_{i_{0}} \times \cdots \times E_{i_{r}}$ and $\varphi_{\alpha}:=\varphi_{i_{0}} \times \widehat{z} \cdots \times \widehat{Z} \varphi_{i_{r}}: U_{i_{0}} \cap \cdots \cap U_{i_{r}} \rightarrow \widehat{Z} \times V_{i_{0}} \times \cdots \times V_{i_{r}}$. Here $U_{i}:=U\left\langle P_{i}\right\rangle, V_{i}:=$ $V\left\langle P_{i}\right\rangle$, and $E_{i}:=E\left\langle P_{i}\right\rangle$. In general, $\varphi_{\alpha}$ is only a quasi-polyhedron. If $\mathscr{N}$ is an abstract simplicial scheme, then $\mathscr{N}^{(k)}, k \in \mathbf{N}$, denotes the subscheme of $\mathscr{N}$ consisting only of simplices of dimension $\leq k$.

(5.4) Let $\mathscr{P}$ be a family of polyhedrons for $\mathscr{X} / T$ with nerve $\mathscr{N}$. We say that $\mathscr{P}$ satisfies the polyhedral axiom if the following two conditions are fulfilled:

(5.4.1) There is a sequence $t=\left(t_{i}\right)_{i \in I}$ of numbers in $] 0,1[$, such that $\operatorname{Ner}\left(\mathscr{P}^{(t)}\right)=\operatorname{Ner}(\mathscr{P})$.

(5.4.2) For $\left\{i_{0}, i_{1}\right\}$ in $\mathscr{N}^{(1)} \backslash \mathscr{N}^{(0)}$, one has $P_{i_{0}} \cup P_{i_{1}} \subset U_{i_{0}} \cap U_{i_{1}}$.

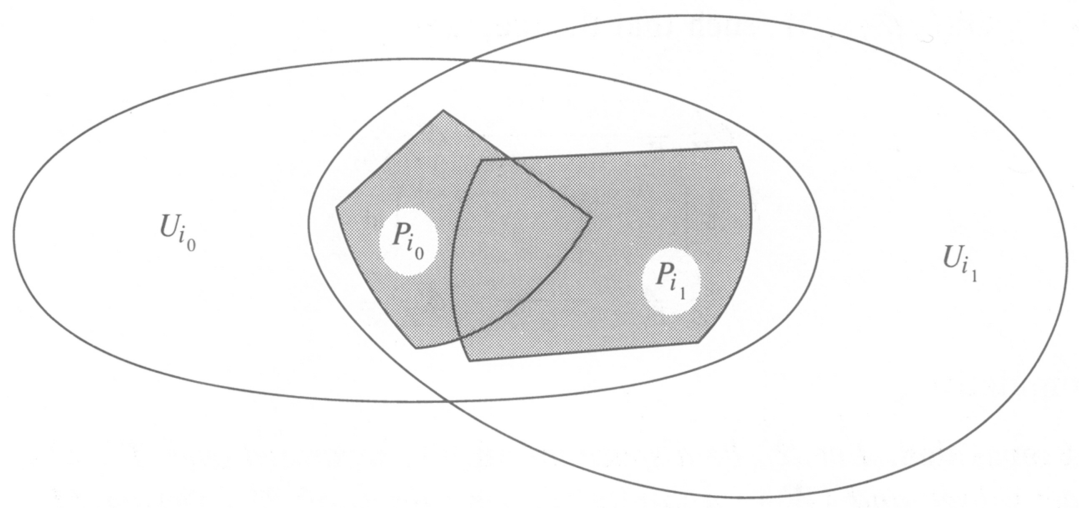

(5.5) Assume that $\mathscr{P}$ satisfies the polyhedral axiom. We put $\widetilde{I \times I}:=$ $\left\{\left(i_{0}, i_{1}\right) \in I \times I:\left\{i_{0}, i_{1}\right\} \in \mathscr{N}^{(1)} \backslash \mathcal{N}^{(0)}\right\}$. A change of charts for $\mathscr{P}$ consists by definition of a family $E_{i}^{\vee}, i \in I$, of open polydiscs in $\mathbf{C}^{n_{i}}$ (with center $x_{i}$ of $E_{i}$, multiradius $b^{i}=\left(b_{1}^{i}, \ldots, b_{n_{i}}^{i}\right)$, and $\left.E_{i} \subset \subset E_{i}^{\vee}\right)$, together with $\hat{Z}$ morphisms

$$
\varphi^{\left(i_{0}, i_{1}\right)}=\left(\operatorname{pr}_{1}, \psi^{\left(i_{0}, i_{1}\right)}\right): \hat{Z} \times \bar{E}_{i_{1}} \rightarrow \widehat{Z} \times \bar{E}_{i_{0}}^{\vee}
$$

for $\left(i_{0}, i_{1}\right) \in \widetilde{I \times I}$, such that $\varphi_{i_{0}}\left(P_{i_{1}}\right) \subset\{0\} \times \bar{E}_{i_{0}}^{\vee}$ and the diagrams

$$
\begin{array}{ccc}
\hat{Z} \times \bar{E}_{i_{1}} \stackrel{\varphi_{i_{1}} \stackrel{\left(i_{0}, i_{1}\right)}{\longrightarrow}}{\longrightarrow} & \widehat{Z} \times \bar{E}_{i_{0}}^{\vee} \\
& P_{i_{1}} & \nearrow \varphi_{i_{0}}
\end{array}
$$

commute. Furthermore, there should be a number $q \in] 0,1[$ with

$$
\sup \left\{\left\|\left(\psi_{j}^{\left(i_{0}, i_{1}\right)} \mid\{0\} \times E_{i_{1}}\right)-x_{j}^{i_{0}}\right\|_{\lambda}: \lambda \in\right] 0,1[\} \leq q b_{j}^{i_{0}}, 3
$$

for $1 \leq j \leq n_{i_{0}}$ and $\left(i_{0}, i_{1}\right) \in \widetilde{I \times I}$.

\footnotetext{
${ }^{3}$ Here we use the $\|\cdot\|_{\lambda}$-norms of the appendix.
} 
If the index set $I$ is finite, we can always find a change of charts for $\mathscr{P}$.

(5.6) Let $\mathscr{P}$ be a family of polyhedrons for $\mathscr{X} / T$, satisfying the polyhedral axiom, together with a change of charts. We call $\mathscr{P}$ special if a total ordering on $I$ is fixed and the following properties hold:

(5.6.1) $n=n_{i}$ is independent of $i \in I$,

(5.6.2) $\bar{E}_{i}^{\vee} \subset V_{i}$ for all $i \in I$,

(5.6.3) the $\varphi^{\left(i_{0}, i_{1}\right)}$ can be represented by open embeddings,

(5.6.4) there are a number $l \in \mathbf{N}$ and exact sequences

$$
\mathscr{O}_{\widehat{Z} \times V_{i}}^{l} \stackrel{s_{i}}{\longrightarrow} \mathscr{O}_{\widehat{Z} \times V_{i}} \rightarrow\left(\varphi_{i}\right)_{*}\left(\mathscr{O}_{U_{i}}\right) \rightarrow 0
$$

of $\mathscr{O}_{\hat{\mathbf{Z}} \times V_{i}}$-modules for each $i \in I$,

(5.6.5) if $\{i, a\}$ is in $\mathscr{N}^{(1)} \backslash \mathscr{N}^{(0)}$, there is an invertible matrix $H_{(i, a)}$ in $\Gamma\left(\widehat{Z} \times V_{i}, \operatorname{Gl}\left(l, \mathscr{O}_{\widehat{Z} \times V_{i}}\right)\right)$, such that the diagram

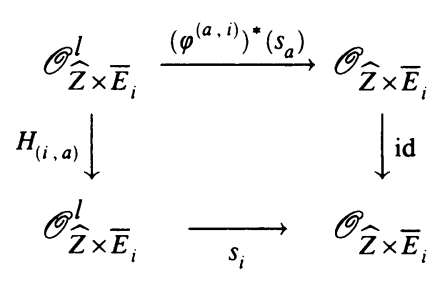

is commutative.

(5.7) Proposition. Let $\mathscr{X}$ be a space in $\operatorname{An}(T)$, separated over $T, M \subset \mathscr{X}$ a compact subset, and $\left(W_{\nu}\right)_{\nu}$ a family of open subsets of $\mathscr{X}$ covering $M$.

Then there is a finite special family $\mathscr{P}$ of polyhedrons for $\mathscr{Z} / T$ and a number $\left.s_{0} \in\right] 0,1[$ with the properties

(a) each $U\left\langle P_{i}\right\rangle$ is contained in some $W_{\nu}$, $M$.

(b) for $t_{i} \in\left[s_{0}, 1\left[\right.\right.$ and $t=\left(t_{i}\right)_{i \in I}$ one has $\operatorname{Ner}\left(\mathscr{P}^{(t)}\right)=\operatorname{Ner}(\mathscr{P})$ and $\left|\mathscr{P}^{(t)}\right| \supset$

Proof. First of all we can find for $\mathscr{X} / T$ a finite family of polyhedrons $\mathscr{Q}=$ $\left(Q_{r}, \varphi_{r}, E_{r}\right)_{r \in R}$ with a shrinking $\mathscr{Q}^{\prime}=\left(Q_{r}^{\prime}, \varphi_{r}, E_{r}^{\prime}\right)_{r \in R}$, such that we have

(1) each $U_{r}$ is contained in some $W_{\nu}$,

(2) $\cup_{r \in R} \dot{Q}_{r}^{\prime} \supset M$

(3) $n=n_{r}$ is independent of $r \in R$,

(4) there are open Stein subsets $E_{\left(r_{0}, r_{1}\right)} \subset E_{r_{0}}$ for $\left\{r_{0}, r_{1}\right\} \in \operatorname{Ner}(\mathscr{Q}), r_{0} \neq r_{1}$, and $\hat{Z}$-isomorphisms

$$
\varphi^{\left(r_{0}, r_{1}\right)}: \hat{Z} \times E_{\left(r_{1}, r_{0}\right)} \rightarrow \hat{Z} \times E_{\left(r_{0}, r_{1}\right)}
$$

with $\varphi_{r_{0}}^{-1}\left(\{0\} \times E_{\left(r_{0}, r_{1}\right)}\right)=\dot{Q}_{r_{0}} \cap \dot{Q}_{r_{1}}$ and $\varphi^{\left(r_{0}, r_{1}\right)} \circ \varphi_{r_{1}}\left|\dot{Q}_{r_{1}} \cap \dot{Q}_{r_{0}}=\varphi_{r_{0}}\right| \dot{Q}_{r_{0}} \cap \dot{Q}_{r_{1}}$,

(5) there are a number $l \in \mathbf{N}$ and exact sequences

$$
\mathscr{O}_{\widehat{Z} \times V_{r}}^{l} \stackrel{s_{r}}{\longrightarrow} \mathscr{O}_{\widehat{Z} \times V_{r}} \rightarrow\left(\varphi_{r}\right)_{*}\left(\mathscr{O}_{U_{r}}\right) \rightarrow 0 \text { for } r \in R
$$


(6) for any $\left\{r_{0}, r_{1}\right\} \in \operatorname{Ner}(\mathscr{Q}), r_{0} \neq r_{1}$, there is an invertible $l \times l$-matrix $H_{\left(r_{0}, r_{1}\right)}$ on $\widehat{Z} \times E_{\left(r_{0}, r_{1}\right)}$, which makes the diagram

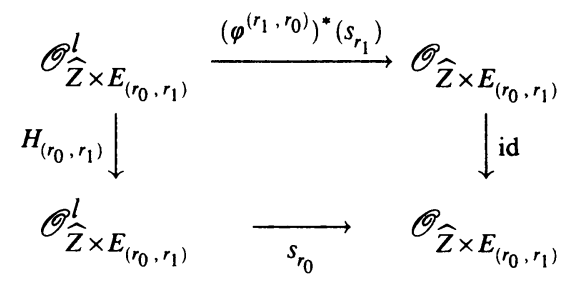

commutative.

The existence of such polyhedron families $\mathscr{Q}$ is proven exactly as in [Fo- $\mathrm{Kn}$, $\S 5]$, using a trick of $\mathrm{H}$. Cartan.

Now we fix open Stein subsets $E_{\left(r_{0}, r_{1}\right)}^{\prime} \subset \subset E_{\left(r_{0}, r_{1}\right)}$ with $\varphi_{r_{0}}^{-1}\left(\{0\} \times E_{\left(r_{0}, r_{1}\right)}^{\prime}\right)=$ $\dot{Q}_{r_{0}}^{\prime} \cap \dot{Q}_{r_{1}}^{\prime}$ and such that $\varphi^{\left(r_{0}, r_{1}\right)}: \hat{Z} \times E_{\left(r_{1}, r_{0}\right)}^{\prime} \rightarrow \widehat{Z} \times E_{\left(r_{0}, r_{1}\right)}^{\prime}$ is a well-defined isomorphism. We are going to refine $\mathscr{Q}$ to a family $\mathscr{P}=\left(P_{i}, \varphi_{i}, E_{i}\right)_{i \in I}$ with refinement map $\tau: I \rightarrow R$, such that $E_{i} \subset E_{\tau(i)}$ and $\varphi_{i}, \varphi^{\left(i_{0}, i_{1}\right)}, H_{\left(i_{0}, i_{1}\right)}$ are induced by $\varphi_{\tau(i)}, \varphi^{\left(\tau\left(i_{0}\right), \tau\left(i_{1}\right)\right)}, H_{\left(\tau\left(i_{0}\right), \tau\left(i_{1}\right)\right)}$.

We set $J_{r}:=\left|\varphi_{r}\left(Q_{r}^{\prime}\right)\right|, r \in R$, and fix a metric on $\mathscr{Z}$. Around each point $z \in J_{r}$ we put a polydisc $E_{z}$, whose diameter is so small that the following conditions are satisfied with $\left(P_{z}:=\varphi_{r}^{-1}\left(\{0\} \times \bar{E}_{z}\right)\right)$ :

(I) diameter $\left(P_{z}\right)<\frac{1}{3} \min \left\{\operatorname{dist}\left(Q_{s}^{\prime}, \complement Q_{s}\right): s \in R\right\}$,

(II) if $z \in E_{\left(r, r_{1}\right)},\left(r, r_{1}\right) \in \operatorname{Ner}(\mathscr{Q})$, then $\bar{E}_{z} \subset E_{\left(r, r_{1}\right)}$,

(III) for $\varphi^{\left(r_{1}, r\right)}=\left(\mathrm{pr}_{1}, \psi^{\left(r_{1}, r\right)}\right): \hat{Z} \times E_{\left(r, r_{1}\right)} \rightarrow \hat{Z} \times E_{r_{1}}$, the estimate

$$
\sup \left\{\left\|\left(\psi_{j}^{\left(r_{1}, r\right)} \mid\{0\} \times E_{z}\right)-x_{j}^{r_{1}}\right\|_{\lambda}: \lambda \in\right] 0,1[\} \leq q_{z} b_{j}^{r_{1}}
$$

holds for some $\left.q_{z} \in\right] 0,1\left[\right.$, all $\left(r, r_{1}\right) \in \widetilde{R \times R}$ and $1 \leq j \leq n$ (here $x^{r_{1}}$ is the center and $b^{r_{1}}$ the multiradius of $E_{r_{1}}$ ).

There is a finite subset $I_{r} \subset J_{r}$ with $\bigcup_{z \in I_{r}} E_{z} \supset \varphi_{r}\left(Q_{r}^{\prime}\right)$. We put $I:=$ $\amalg_{r} I_{r}$ and define $\tau: I \rightarrow R$ by $\tau \mid I_{r}:=r$, as well as $\varphi_{i}:=\varphi_{\tau(i)}, U_{i}:=U_{\tau(i)}$, $V_{i}:=V_{\tau(i)}$. This gives a family $\mathscr{P}=\left(P_{i}, \varphi_{i}, E_{i}\right)_{i \in I}$ of polyhedrons for $\mathscr{X} / T$, satisfying condition (5.4.2) (because of (I)). By an (arbitrary) small change of the multi-radii of the $E_{i}$, we may assume that $\mathscr{P}$ fulfills the polyhedral axiom (compare [B-K, (IV.3.7)]). We still must specify the change of charts for $\mathscr{P}$. For this we set $E_{i}^{\vee}:=E_{\tau(i)}$. If $\left(i_{0}, i_{1}\right)$ is in $\widetilde{I \times I}$ with $\tau\left(i_{0}\right)=\tau\left(i_{1}\right)$, then $E_{i_{0}} \cup E_{i_{1}} \subset E_{\tau\left(i_{0}\right)}=E_{\tau\left(i_{1}\right)}$ and $\varphi^{\left(i_{0}, i_{1}\right)}$ shall be induced by $\operatorname{id}_{\widehat{Z} \times E_{\tau\left(i_{0}\right)}}$.

If $\tau\left(i_{0}\right) \neq \tau\left(i_{1}\right)$, then $\left\{\tau\left(i_{0}\right), \tau\left(i_{1}\right)\right\} \in \operatorname{Ner}(\mathscr{Q})$ and $P_{i_{0}} \cup P_{i_{1}} \subset \dot{Q}_{\tau\left(i_{0}\right)} \cap \dot{Q}_{\tau\left(i_{1}\right)}$ according to (I). The center of $E_{i_{0}}$, resp. $E_{i_{1}}$, lies automatically in $E_{\left(\tau\left(i_{0}\right), \tau\left(i_{1}\right)\right)}$, resp. $E_{\left(\tau\left(i_{1}\right), \tau\left(i_{0}\right)\right)}$. By (II) we have $\bar{E}_{i_{1}} \subset E_{\left(\tau\left(i_{1}\right), \tau\left(i_{0}\right)\right)}$ and therefore we can define $\varphi^{\left(i_{0}, i_{1}\right)}:=\varphi^{\left(\tau\left(i_{0}\right), \tau\left(i_{1}\right)\right)} \mid \hat{Z} \times \bar{E}_{i_{1}}$. This is the desired change of charts. 
At last, we define $H_{\left(i_{0}, i_{1}\right)}:=H_{\left(\tau\left(i_{0}\right), \tau\left(i_{1}\right)\right)} \mid \hat{Z} \times \bar{E}_{i_{0}}$ for $\left\{i_{0}, i_{1}\right\} \in \operatorname{Ner}(\mathscr{P})$, $i_{0} \neq i_{1}$. Obviously, these data have the desired properties and so (5.7) is proved.

(5.8) Let $\mathscr{P}$ be a special family of polyhedrons for $\mathscr{X} / T$ with nerve $\mathscr{N}$ and put $J:=\{1, \ldots, l\}$. We define

$$
\mathscr{R}_{i}^{0}:=\mathscr{O}_{\bar{E}_{i}}, \quad \mathscr{R}_{i}^{-1}:=\coprod_{j \in J} \mathscr{O}_{\bar{E}_{i}} e_{i, j}
$$

with free generators $e_{i, j}$ of degree -1 , and for $\alpha=(i, a) \in \mathscr{N}, i<a$, we set

$$
\begin{aligned}
& \mathscr{R}_{\alpha}^{0}:=\mathscr{O}_{\bar{E}_{\alpha}}, \\
& \mathscr{R}_{\alpha}^{-1}:=\coprod_{j \in J} \mathscr{O}_{\bar{E}_{\alpha}} e_{i, j} \amalg \coprod_{j \in J} \mathscr{O}_{\bar{E}_{\alpha}} e_{a, j} \amalg \coprod_{1 \leq j \leq n} \mathscr{O}_{\bar{E}_{\alpha}} e_{\alpha, j}
\end{aligned}
$$

with further free generators $e_{\alpha, j}$ of degree -1 for $j \in\{1, \ldots, n\}$. Moreover, we put $\mathscr{R}_{i, Z}^{0}:=\mathscr{O}_{Z \times \bar{E}_{i}}, \mathscr{R}_{i, \widehat{Z}}^{0}:=\mathscr{O}_{\widehat{Z} \times \bar{E}_{i}}$ and analogously $\mathscr{R}_{i, Z}^{-1}$, etc. The morphism $s_{i}: \mathscr{R}_{i, \widehat{Z}}^{-1} \rightarrow \mathscr{R}_{i, \hat{Z}}^{0}$ is taken from (5.6.4) and

$$
s_{\alpha}: \mathscr{R}_{\alpha, \widehat{Z}}^{-1} \rightarrow \mathscr{R}_{\alpha, \widehat{Z}}^{0}
$$

is given by $s_{\alpha}\left(e_{i, j}\right):=s_{i}\left(e_{i, j}\right), s_{\alpha}\left(e_{a, j}\right):=s_{a}\left(e_{a, j}\right)$ and $s_{\alpha}\left(e_{\alpha, j}\right):=z_{j}^{a}-\psi_{j}^{(a, i)}$, where $z_{j}^{a}$ is the $j$ th coordinate function on $\bar{E}_{a}$ for $1 \leq j \leq n$. One easily shows

(5.8.1) Lemma. $\operatorname{Coker}\left(s_{\alpha}\right)=\left(\left(\varphi_{\alpha}\right)_{*}\left(\mathscr{O}_{\mathscr{Z}} \mid U_{\alpha}\right)\right) \mid \widehat{Z} \times \bar{E}_{\alpha}$.

Let $H_{(i, a)}$ be as in (5.6.5) and $H_{(i, a)}\left(e_{a, j}\right)=\sum_{j^{\prime}} h_{j, j^{\prime}}^{(a)} e_{i, j^{\prime}}$. We set

$$
\xi_{j}:=\left(\sum_{j^{\prime}=1}^{l} h_{j, j^{\prime}}^{(a)} s_{\alpha}\left(e_{i, j^{\prime}}\right)\right)-s_{\alpha}\left(e_{a, j}\right),
$$

considered as an element in $\Gamma\left(\widehat{Z} \times \bar{E}_{i} \times \bar{E}_{a}^{\vee}, \mathscr{O}_{\widehat{Z} \times \mathbf{C}^{n} \times \mathbf{C}^{n}}\right)$.

(5.8.2) Lemma. There are $\tilde{h}_{j, k} \in \Gamma\left(\hat{Z} \times \bar{E}_{i} \times \bar{E}_{a}^{\vee}, \mathscr{O}_{\widehat{Z} \times \mathbf{C}^{n} \times \mathbf{C}^{n}}\right)$ for $1 \leq j \leq l$, $1 \leq k \leq n$ with

$$
\xi_{j}+\sum_{k=1}^{n} \tilde{h}_{j, k} \cdot\left(z_{k}^{a}-\psi_{k}^{(a, i)}\right)=0 .
$$

Proof. Let $\Phi^{(a, i)}: \hat{Z} \times \bar{E}_{i} \rightarrow \hat{Z} \times \bar{E}_{i} \times \bar{E}_{a}^{\vee}$ be defined by

$$
\Phi^{(a, i)}:=\left(\mathrm{id}_{\widehat{Z} \times \bar{E}_{i}}, \psi^{(a, i)}\right) .
$$

The commutativity of the diagram in $(5.6 .5)$ gives $\left(\Phi^{(a, i)}\right)^{*}\left(\xi_{j}\right)=0$ for $j \in J$. This implies the lemma. 
(5.8.3) Remark. In the situation of (5.8.2) we put

$$
h_{j}:=-\sum_{j^{\prime}} h_{j, j^{\prime}}^{(a)} e_{i, j^{\prime}}-\sum_{k}\left(\tilde{h}_{j, k} \mid \widehat{Z} \times \bar{E}_{\alpha}\right) e_{\alpha, k} .
$$

Then we get

$$
s_{\alpha}\left(h_{j}\right)+s_{\alpha}\left(e_{a, j}\right)=0
$$

in $\Gamma\left(\widehat{Z} \times \bar{E}_{\alpha}, \mathscr{R}_{\alpha, \widehat{Z}}^{0}\right)$ for $j \in J$.

(5.9) Definition. Let $\mathscr{P}$ be a special family of polyhedrons for $\mathscr{X} / T$. We call $\mathscr{P}$ augmented if for each $(i, a) \in \operatorname{Ner}(\mathscr{P}), i<a$, there are elements $\tilde{h}_{j, k} \in \Gamma\left(\widehat{Z} \times \bar{E}_{\alpha}, \mathscr{O}_{\widehat{Z} \times \bar{E}_{\alpha}}\right), 1 \leq j \leq l, 1 \leq k \leq n$, such that the identity

$$
s_{\alpha}\left(h_{j}\right)+s_{\alpha}\left(e_{a, j}\right)=0, \quad j \in J,
$$

holds with

$$
h_{j}:=-\sum_{j^{\prime}} h_{j, j^{\prime}}^{(a)} e_{i, j^{\prime}}-\sum_{k} \tilde{h}_{j, k} e_{\alpha, k} .
$$

Using (5.8.2), (5.8.3), a special $\mathscr{P}$ is always augmentable.

We have now established the necessary preparations in order to construct special resolvents for the structure sheaf $\mathscr{O}_{\mathscr{L}}$ with respect to a fixed augmented special family of polyhedrons. The basic idea is due to Palamodov $\left[\mathrm{Pa}_{1}\right]$ and this method has been fairly generalized in [B-K].

(5.10) Let $\mathscr{X}$ be as above, $Q \subset \mathscr{X}$ a compact subset and $\mathscr{P}=\left(P_{i}, \varphi_{i}, E_{i}\right)_{i \in I}$ a finite augmented special family of polyhedrons for $\mathscr{X} / T$ with nerve $\mathscr{N}$ and $\left.\left|\mathscr{P}^{\left(s_{0}\right)}\right| \supset Q, s_{0} \in\right] 0,1\left[\right.$ near by 1 . Each $\alpha=\left(i_{0}, \ldots, i_{m}\right)$ in $\mathscr{N}$ is always written in ordered form $i_{0}<i_{1}<\cdots<i_{m}$ and $a(\alpha):=i_{m},|\alpha|:=m$.

For $i \in I$ we fix a free graded anticommutative C-algebra ("free" in the sense of $[\mathrm{B}-\mathrm{K},(\mathrm{I} .7 .8)(2)])$

with the following properties:

$$
R_{i}=\mathbf{C}\left\langle e_{i, j}\right\rangle_{j \in J_{i}}
$$

(1) $J_{i}=\bigsqcup_{k \leq 0} J_{i}^{k}$ with $\operatorname{card}\left(J_{i}^{k}\right)<\infty$ and $\operatorname{deg}\left(e_{i, j}\right)=k \Leftrightarrow j \in J_{i}^{k}$; moreover $J_{i}^{0}=\{1, \ldots, n\}$,

(2) $R_{i}^{0}=\mathrm{C}\left\langle e_{i, j}\right\rangle_{j \in J_{i}^{0}}=\Gamma\left(\bar{E}_{i}, \mathscr{O}_{\bar{E}_{i}}\right)$ and $e_{i, j}$ identifies with the $j$ th coordinate function on $\bar{E}_{i, j}$ for $j \in J_{i}^{0}$,

(3) there is a bijection $\{1, \ldots, l\} \stackrel{\sim}{\rightarrow} J_{i}^{-1}$, such that the generators $e_{i, j}$, $j \in J_{i}^{-1}$, coincide with the generators from (5.8),

(4) on $R_{i, \widehat{Z}}=\bigsqcup_{k} \Gamma\left(\widehat{Z} \times \bar{E}_{i}, \mathscr{R}_{i, \widehat{Z}}^{k}\right)$ there is a derivation $s_{i}$ of degree 1 with $s_{i}^{2}=0$, such that $\left(R_{i, \hat{Z}}, s_{i}\right)$ is a resolvent ${ }^{4}$ of $\Gamma\left(\hat{Z} \times \bar{E}_{i},\left(\varphi_{i}\right)_{*}\left(\mathscr{O}_{\mathscr{Z}} \mid U_{i}\right)\right)$ and $s_{i}: R_{i, \widehat{Z}}^{-1} \rightarrow R_{i, \widehat{Z}}^{0}$ is consistent with the data in (5.6.4).

\footnotetext{
${ }^{4}$ "Resolvent" $=$ resolution of this type.
} 
The simplicial $\mathscr{N}$-algebra $R=\left(R_{\alpha}\right)_{\alpha \in \mathscr{N}}$, given by

$$
R_{\alpha}=\mathbf{C}\left\langle e_{\beta, j}^{\alpha}\right\rangle_{\beta \subset \alpha, j \in J_{a(\beta)}}
$$

with free generators $e_{\beta, j}^{\alpha}$ of degree $\left(\operatorname{deg}\left(e_{\alpha(\beta), j}\right)-|\beta|\right)$ and $R_{\alpha}^{0}=\Gamma\left(\bar{E}_{\alpha}, \mathscr{O}_{\bar{E}_{\alpha}}\right)$ (where the transition maps $r_{\beta}^{\alpha}: R_{\beta} \rightarrow R_{\alpha}$ are induced by $e_{\gamma, j}^{\beta} \mapsto e_{\gamma, j}^{\alpha}$ for $\gamma \subset \beta \subset \alpha)$, is even a free algebra in the simplicial sense:

$$
R=\mathbf{C}\left\langle e_{\alpha, j}^{\alpha}\right\rangle_{\alpha \in \mathcal{N}, j \in J_{a(\alpha)}} .
$$

Observe that $R_{\alpha}$ is consistent with (5.8) for $\alpha=(i, a)$.

On the set of free generators of $R_{\alpha}$, we introduce a function $\psi$ with $\psi\left(e_{\beta, j}^{\alpha}\right)$ $=1$ if $\beta \supset \alpha \backslash a(\alpha)$ and $\psi\left(e_{\beta, j}^{\alpha}\right):=\operatorname{deg}\left(e_{a(\alpha), j}\right)$ otherwise. Obviously, $\psi \leq 1$. For $k \leq 1$ we set

$$
K_{\alpha}^{k}:=\mathbf{C}\left\langle e_{\beta, j}^{\alpha}\right\rangle_{\beta \subset \alpha, j \in J_{\alpha(\beta)}}, \quad \psi\left(e_{\beta, j}^{\alpha}\right) \geq k .
$$

Then $K^{k}=\left(K_{\alpha}^{k}\right)_{\alpha \in \mathcal{N}}$ is a free C-subalgebra of $R$ with $K^{k+1} \subset K^{k}$ and $\bigcup_{k} K^{k}=R$. For $i \in I$ we have $K_{i}^{1}=\mathrm{C}$ and the image of $R_{\alpha \backslash a(\alpha)}$ in $R_{\alpha}$ is just $K_{\alpha}^{1}$ in the case $\alpha \in \mathscr{N} \backslash \mathscr{N}^{(0)}$.

For each $\alpha \in \mathscr{N}$, there is a $R_{\alpha}^{0}$-linear derivation $\partial_{\alpha}: R_{\alpha} \rightarrow R_{\alpha}$ of degree 1 , such that for a free generator $e_{\beta, j}^{\alpha}$ of $R_{\alpha}$ with $\beta=\left(i_{0}, \ldots, i_{m}\right)$, we have $\partial_{\alpha}\left(e_{\beta, j}^{\alpha}\right)=\sum_{k=0}^{m-1}(-1)^{k} e_{\beta \backslash i_{k}, j}^{\alpha}$ in the case $|\beta|=m>0, i_{m}=a(\alpha)$, and $\partial_{\alpha}\left(e_{\beta, j}^{\alpha}\right)=0$ otherwise. One easily checks that $\partial_{\alpha}^{2}=0$ and each $K_{\alpha}^{k}$ is stable under $\partial_{\alpha}$.

(5.11) Definition. An $R_{\widehat{Z}}^{0}$-linear derivation $s$ of degree 1 on $R_{\widehat{Z}}$ is called special if the following conditions are satisfied:

(1) The pair $\left(R_{\widehat{Z}}, s\right)$ is a resolvent of $\Gamma\left(\widehat{Z} \times \bar{E}_{*},\left(\varphi_{*}\right)_{*}\left(\mathscr{O}_{\mathscr{Z}} \mid U_{*}\right)\right)$ and $s \mid R_{i, \widehat{Z}}=$ $s_{i}$ for $i \in \mathscr{N}^{(0)}$.

(2) $s$ stabilizes the (obviously defined) filtration $K_{\widehat{Z}}^{\cdot}$ of $R_{\widehat{Z}}$. For a free generator $e_{\beta, j}^{\alpha}$ of $R_{\alpha}$ with $\psi\left(e_{\beta, j}^{\alpha}\right) \geq k \leq 0$, the element $\left(s-\partial_{\alpha}\right)\left(e_{\beta, j}^{\alpha}\right)$ is in $K_{\alpha, \widehat{Z}}^{k+1}$.

(3) For $\alpha=(i, a) \in \mathscr{N}^{(1)}$, the restriction $s \mid R_{\alpha, \widehat{Z}}^{-1}$ coincides with the appropriate homomorphism in (5.8).

(4) If $\alpha=(i, a) \in \mathscr{N}^{(1)}$, then $s\left(e_{\alpha, j}^{\alpha}\right)=e_{a(\alpha), j}+h_{j}$ for $j \in J_{a(\alpha)}^{-1}$, where $h_{j}$ is given by the formula in (5.9). Observe that $h_{j} \in K_{\alpha, \hat{z}}^{0}$.

(5.12) Theorem. Let $\mathscr{X}, Q, \mathscr{P}$, and $R$ be as in (5.10). Then there exists a special derivation $s$ on $R_{\widehat{Z}}$. (Such a resolvent $\left(R_{\widehat{Z}}, s\right)$ will be called special for short.)

The proof of the corresponding result in [B-K, Kap. IV, Satz 1.7] also applies to this context. The only modification is that the very last step in the proof of 
[B-K, (IV. 1.7.3)] is taken for granted here, since $\mathscr{P}$ is augmented by the $h_{j}$.

\section{SPECIAL RESOlutions of COHERENT MODUles}

In this section we are going to make a similar construction as in $\S 5$, but now for the case of modules. We start with the following situation:

(6.1) Let $S$ be a smooth complex space germ (considered here always as a formal space germ, so that the underlying set is just one point), $X$ a space in $\operatorname{An}(S)$ with separated structure map $X \rightarrow S$, and $T=(0, A)$ a formal space germ over $S$. Moreover, we fix a module $\mathscr{F}$ in $\operatorname{Coh}_{p}\left(X_{A}\right)$ and assume that the following data are given:

(6.1.1) a finite augmented special family of polyhedrons $\mathscr{P}=\left(P_{i}, \varphi_{i}, E_{i}\right)_{i \in I}$ for $X / S$ with nerve $\mathcal{N}$ and a smooth complex space germ $Z^{\prime}$ together with an $S$-embedding $T \rightarrow \hat{Z}$, where $Z=S \times Z^{\prime}$ and the completion $\hat{Z}$ is taken with respect to some idal $\mathfrak{a} \subset \mathscr{O}_{Z}$, such that

$$
\left|\mathscr{P}_{\widehat{Z}}^{\left(t_{0}\right)}\right| \supset \operatorname{supp}(F) .^{5}
$$

Here $\mathscr{P}_{\widehat{Z}}$ is the base changed family of polydiscs for $X_{\widehat{Z}} / \widehat{Z}$ and $t_{0}$ is a number in $] 0,1[$ near by 1 ;

(6.1.2) a special resolvent $\left(R_{S}, s\right)$ of $X / S$ with respect to $\mathscr{P}$;

(6.1.3) finite disjoint index sets $J^{0}(\mathscr{F}), J^{-1}(\mathscr{F})$ and exact sequences

$$
\coprod_{j \in J^{-1}(\mathscr{F})} \mathscr{O}_{V_{i, \widehat{z}}} m_{i, j} \stackrel{s_{i}}{\longrightarrow} \coprod_{j \in J^{0}(\mathscr{F})} \mathscr{O}_{V_{i, \widehat{z}}} m_{i, j} \rightarrow\left(\varphi_{i, \widehat{z}}\right)_{*}\left(\mathscr{F} \mid U_{i, \widehat{z}}\right) \rightarrow 0
$$

with free generators $m_{i, j}$;

(6.1.4) for each $\left\{i_{0}, i_{1}\right\} \in \mathscr{N}^{(1)} \backslash \mathscr{N}^{(0)}$ commutative diagrams of $\mathscr{O}_{\bar{E}_{i_{0}}, \bar{z}}-$ modules

$$
\begin{aligned}
& \amalg_{j \in J^{-1}(\mathscr{F})} \mathscr{O}_{\bar{E}_{i_{0}}, \widehat{z}} m_{i_{1}, j} \stackrel{\left(\varphi_{\bar{Z}}^{\left(i_{1}, i_{0}\right)}\right)^{*}\left(s_{i_{1}}\right)}{\longrightarrow} \amalg_{j \in J^{0}(\mathscr{F})} \mathscr{O}_{\bar{E}_{i_{0}, \widehat{z}}} m_{i_{1}, j} \\
& \tilde{\Phi}_{\left(i_{0}, i_{1}\right)}^{(-1)} \downarrow l \quad l \mid \Phi_{\left(i_{0}, i_{1}\right)}^{(0)} \\
& \amalg_{j \in J^{-1}(\mathscr{F})} \mathscr{O}_{\bar{E}_{i_{0}, \widehat{z}}} m_{i_{0}, j} \quad \underset{s_{i_{0}}}{\longrightarrow} \quad \amalg_{j \in J^{0}(\mathscr{F})} \mathscr{Q}_{\bar{E}_{i_{0}, \widehat{z}}} m_{i_{0}, j}
\end{aligned}
$$

with isomorphisms $\Phi_{\left(i_{0}, i_{1}\right)}^{(0)}, \tilde{\Phi}_{\left(i_{0}, i_{1}\right)}^{(-1)}$.

The existence of such data is easily seen, compare the proof of (5.7).

(6.2) On $V_{i}$ we put

$$
\begin{aligned}
& \mathscr{M}_{i}^{0}:=\coprod_{j \in J^{0}(\mathscr{F})} \mathscr{R}_{i}^{0} m_{i, j}, \\
& \mathscr{M}_{i}^{-1}:=\coprod_{j \in J^{-1}(\mathscr{F})} \mathscr{R}_{i}^{0} m_{i, j} \amalg \coprod_{j \in J^{0}(\mathscr{F})} \mathscr{R}_{i}^{-1} m_{i, j},
\end{aligned}
$$

${ }^{5} \mathscr{F}$ is considered as an $\mathscr{O}_{X_{\widehat{Z}}}$-module via the embedding $X_{T} \hookrightarrow X_{\widehat{Z}}$. 
and define the homomorphism

$$
s_{i}: \mathscr{M}_{i, \widehat{Z}}^{-1} \rightarrow \mathscr{M}_{i, \widehat{Z}}^{0}
$$

on $V_{i, \widehat{z}}$ via the product rule $s_{i}\left(a m_{i, j}\right)=s_{i}(a) m_{i, j}+(-1)^{d(a)} a s_{i}\left(m_{i, j}\right)$ where $d(a)$ is the degree of $a$. For $\{i, a\} \in \mathscr{N}^{(1)} \backslash \mathscr{N}^{(0)}$, we have the following commutative diagram

$$
\begin{aligned}
& \left(\varphi_{\widehat{Z}}^{(a, i)}\right)^{*}\left(\mathscr{M}_{a, \widehat{Z}}^{-1} \mid \bar{E}_{a, \widehat{Z}}^{\vee}\right) \stackrel{\left(\varphi_{\widehat{Z}}^{(a, i)}\right)^{*}\left(s_{a}\right)}{\longrightarrow}\left(\varphi_{\widehat{Z}}^{(a, i)}\right)^{*}\left(\mathscr{M}_{a, \widehat{Z}}^{0} \mid \bar{E}_{a, \widehat{Z}}^{\vee}\right) \\
& \boldsymbol{\Phi}_{(i, a)}^{(-1)} \downarrow l \quad l \downarrow \Phi_{(i, a)}^{(0)} \\
& \mathscr{M}_{i, \widehat{Z}}^{-1} \quad \underset{s_{i}}{\longrightarrow} \quad \mathscr{M}_{i, \hat{Z}}^{0}
\end{aligned}
$$

of $\mathscr{O}_{\bar{E}_{i, \widehat{z}}}$-modules with an isomorphism $\Phi_{(i, a)}^{(-1)}$ defined by

$$
\begin{gathered}
\Phi_{(i, a)}^{(-1)}\left(m_{a, j}\right):=\tilde{\Phi}_{(i, a)}^{(-1)}\left(m_{a, j}\right), \quad j \in J^{-1}(\mathscr{F}), \\
\Phi_{(i, a)}^{(-1)}\left(e_{a, k} m_{a, j}\right):=H_{(i, a)}\left(e_{a, k}\right) \Phi_{(i, a)}^{(0)}\left(m_{a, j}\right), \quad j \in J^{0}(\mathscr{F}),
\end{gathered}
$$

where $e_{a, k}$ is a free generator of $R_{a}$ of degree -1 . The data (6.1.1)-(6.1.4) together with $\Phi^{(0)}, \Phi^{(-1)}$ are called a special polyhedron family for $(X / S ; \mathscr{F})$.

(6.3) Let $\alpha=(i, a)$ be in $\mathscr{N}$ with $i<a$. On $\bar{E}_{\alpha, \widehat{z}}^{\vee}$ we set

$$
\begin{aligned}
\mathscr{M}_{\alpha}^{0}:= & \coprod_{j \in J^{0}(\mathscr{F})} \mathscr{R}_{\alpha}^{0} m_{i, j} \amalg \coprod_{j \in J^{0}(\mathscr{F})} \mathscr{R}_{\alpha}^{0} m_{a, j}, \\
\mathscr{M}_{\alpha}^{-1}:= & \coprod_{j \in J^{-1}(\mathscr{F})} \mathscr{R}_{\alpha}^{0} m_{i, j} \amalg \coprod_{j \in J^{-1}(\mathscr{F})} \mathscr{R}_{\alpha}^{0} m_{a, j} \amalg \coprod_{j \in J^{0}(\mathscr{F})} \mathscr{R}_{\alpha}^{-1} m_{i, j} \\
& \amalg \coprod_{j \in J^{0}(\mathscr{F})} \mathscr{R}_{\alpha}^{-1} m_{a, j} \amalg \coprod_{j \in J^{0}(\mathscr{F})} \mathscr{R}_{\alpha}^{0} m_{\alpha, j}
\end{aligned}
$$

with new free generators $m_{\alpha, j}$ and define $s_{\alpha}: \mathscr{M}_{\alpha, \widehat{Z}}^{-1} \rightarrow \mathscr{M}_{\alpha, \widehat{Z}}^{0}$ on $\left(\bar{E}_{i} \times \bar{E}_{a}^{\vee}\right) \widehat{Z}$ by

$$
\begin{aligned}
& s_{\alpha}\left(m_{\nu, j}\right):=s_{\nu}\left(m_{\nu, j}\right), \quad \nu \in\{i, a\}, \\
& s_{\alpha}\left(m_{\alpha, j}\right):=m_{a, j}-\Phi_{(i, a)}^{(-1)}\left(m_{a, j}\right), \quad j \in J^{0}(\mathscr{F}),
\end{aligned}
$$

such that the product rule holds again. One easily shows

(6.3.1) Lemma. $\operatorname{Coker}\left(s_{\alpha}\right)=\left(\left(\varphi_{\alpha, \widehat{Z}}\right)_{*}\left(\mathscr{F} \mid U_{\alpha, \widehat{Z}}\right)\right)$ on $\bar{E}_{\alpha, \widehat{Z}}$.

(6.4) Let $\alpha=(i, a)$ be in $\mathscr{N}^{(1)}, i<a$, and

$$
\boldsymbol{\Phi}_{(i, a)}^{(-1)}\left(m_{a, j}\right)=\sum_{j^{\prime} \in J^{-1}(\mathscr{F})} h_{j, j^{\prime}}^{(\mathscr{F})} m_{i, j^{\prime}}+\sum_{k \in J^{0}(\mathscr{F})} g_{j, k} m_{i, k}
$$


for $j \in J^{-1}(\mathscr{F})$. Analogously to (5.8), we now put

$$
\xi_{j}^{(\mathscr{F})}:=\sum_{j^{\prime} \in J^{-1}(\mathscr{F})} h_{j, j^{\prime}}^{(\mathscr{F})} s_{\alpha}\left(m_{i, j^{\prime}}\right)+\sum_{k \in J^{0}(\mathscr{F})} s_{\alpha}\left(g_{j, k}\right) m_{i, k}-s_{\alpha}\left(m_{a, j}\right)
$$

in $\Gamma\left(\left(\bar{E}_{i} \times \bar{E}_{a}\right)_{\widehat{Z}}, \mathscr{M}_{\alpha, \widehat{Z}}^{0}\right)$. We denote by $\Gamma^{(a, i)}$ the graph-mapping over $\widehat{Z}$ associated to the $\hat{Z}$-morphism

$$
\left(\varphi^{(a, i)}, \Phi_{(i, a)}^{(0)}\right):\left(\bar{E}_{i, \widehat{Z}}, \mathscr{O}_{\widehat{E}_{i, \widehat{z}}}\left[\mathscr{M}_{i, \widehat{Z}}^{0}\right]\right) \rightarrow\left(\bar{E}_{a, \widehat{Z}}^{\vee}, \mathscr{O}_{\bar{E}_{a, \widehat{Z}}^{\vee}}\left[\mathscr{M}_{a, \widehat{Z}}^{0}\right]\right) .
$$

Here [*] means idealization with respect to $*$. The commutativity of $(6.2 .2)$ immediately gives

$$
\left(\Gamma^{(a, i)}\right)^{*}\left(\xi_{j}\right)=0
$$

so there are elements $\tilde{h}_{j, k}^{(\mathscr{F})}, c_{j, k, r}^{(\nu)}$ in $\Gamma\left(\left(\bar{E}_{i} \times \bar{E}_{a}^{\vee}\right)_{\widehat{Z}}, \bar{E}_{\alpha, \widehat{Z}}^{\vee}\right)$ for $k \in J^{0}(\mathscr{F})$, $\nu \in\{i, a\}, 1 \leq r \leq n$ such that

$$
\xi_{j}^{(\mathscr{F})}+\sum_{k} \tilde{h}_{j, k}^{(\mathscr{F})}\left(m_{a, k}-\Phi_{(i, a)}^{(0)}\left(m_{a, k}\right)\right)+\sum_{k, \nu, r} c_{j, k, r}^{(\nu)}\left(z_{r}^{a}-\psi_{r}^{(a, i)}\right) m_{\nu, k}=0 .
$$

If we define

$$
h_{j}^{(\mathscr{F})}:=-\Phi_{(i, a)}^{(-1)}\left(m_{a, j}\right)-\sum_{k} \tilde{h}_{j, k}^{(\mathscr{F})} m_{\alpha, k}-\sum_{k, \nu, r} c_{j, k, r}^{(\nu)} e_{\alpha, r} m_{\nu, k}
$$

on $\bar{E}_{\alpha, \widehat{Z}}$, then we get

$$
s_{\alpha}\left(h_{j}^{(\mathscr{F})}\right)+s_{\alpha}\left(m_{a, j}\right)=0 .
$$

Therefore the following definition makes sense.

(6.5) Definition. A special family of polyhedrons $\mathscr{P}$ for $(X / S ; \mathscr{F})$ is called augmented if for each $\alpha=(i, a) \in \operatorname{Ner}(\mathscr{P}), i<a$, there are elements $\tilde{h}_{j, k}^{(\mathscr{F})}$, $c_{j, k, r}^{(\nu)}$ in $\Gamma\left(\bar{E}_{\alpha, \widehat{Z}}, \mathscr{O}_{\bar{E}_{\alpha, \widehat{z}}}\right)$ for $j \in J^{-1}(\mathscr{F}), k \in J^{0}(\mathscr{F}), r \in\{1, \ldots, n\}$, $\nu \in\{i, a\}$, such that

$$
s_{\alpha}\left(h_{j}^{(\mathscr{F})}\right)+s_{\alpha}\left(m_{a, j}\right)=0
$$

where $h_{j}^{(\mathscr{F})}$ is defined by the formula (6.4.1).

(6.6) We fix an augmented special family of polyhedrons $\mathscr{P}$ for $(X / S ; \mathscr{F})$ with nerve $\mathscr{N}$ and a free graded $R_{i}$-module $M_{i}=\bigsqcup_{j \in J_{i}(\mathscr{F})} R m_{i, j}$ for $i \in I$ with the following properties:

(1) $J_{i}(\mathscr{F})=\bigsqcup_{g \leq 0} J_{i}^{g}(\mathscr{F})$ with $\operatorname{card}\left(J_{i}^{g}(\mathscr{F})\right)<\infty$ and $\operatorname{deg}\left(m_{i, j}\right)=g \Leftrightarrow j \in$ $J_{i}^{g}(\mathscr{F})$; moreover $J_{i}^{0}(\mathscr{F})=J^{0}(\mathscr{F}), J_{i}^{-1}(\mathscr{F})=J^{-1}(\mathscr{F})$ and the generators $m_{i, j}$ are identified with those in (6.1).

(2) $M_{i, \widehat{Z}}$ has a differential $s_{i}$ of degree 1 which fulfills the product rule, such that $\left(M_{i, \widehat{Z}}, s_{i}\right)$ is a resolution of $\Gamma\left(\bar{E}_{i, \widehat{Z}},\left(\varphi_{i, \widehat{Z}}\right)_{*}\left(\mathscr{F} \mid U_{i, \widehat{Z}}\right)\right)$; moreover $s_{i}$ is consistent with (6.1.3). The simplicial $R$-module $M=\left(M_{\alpha}\right)_{\alpha \in \mathcal{N}}$ with

$$
M_{\alpha}=\coprod_{\substack{\beta \subset \alpha \\ j \in J_{\alpha(\beta)}(\mathscr{F})}} R_{\alpha} m_{\beta, j}^{\alpha}
$$


$\operatorname{deg}\left(m_{\beta, j}^{\alpha}\right)=\operatorname{deg}\left(m_{a(\beta), j}\right)-|\beta|$, and where $M_{\beta} \rightarrow M_{\alpha}$ is induced by $m_{\gamma, j}^{\beta} \mapsto$ $m_{\gamma, j}^{\alpha}$ for $\gamma \subset \beta \subset \alpha$, is a free $R$-module in the simplicial sense:

$$
M=\coprod_{\substack{\alpha \in \mathcal{N}, j \in J_{a(\alpha)}(\mathscr{F})}} R m_{\alpha, j}^{\alpha} .
$$

Observe that $M_{(i, a)}^{-1}$ identifies canonically with $\Gamma\left(\bar{E}_{(i, a)}, \mathscr{M}_{(i, a)}^{-1}\right)$ from (6.3). Analogously to (5.10), a $\psi$-function is defined on the free generators $m_{\beta, j}^{\alpha}$ of $M_{\alpha}$. We set here

$$
K_{\alpha}^{k}(\mathscr{F}):=\coprod_{\beta \subset \alpha} K_{\alpha}^{k} m_{\beta, j}^{\alpha}, \quad j \in J_{a(\beta)}(\mathscr{F}), \quad \psi\left(m_{\beta, j}^{\alpha}\right) \geq k,
$$

for $k \leq 1$. Then $K^{k}(\mathscr{F}):=\left(K_{\alpha}^{k}(\mathscr{F})\right)_{\alpha \in \mathcal{N}}$ is a free graded $K^{k}$-module with $K^{k+1}(\mathscr{F}) \subset K^{k}(\mathscr{F})$ and $\bigcup_{k} K^{k}(\mathscr{F})=M$.

Let $\alpha$ be in $\mathscr{N}$. Then there exists a unique $R_{\alpha, \widehat{Z}^{0}}^{0}$ linear homomorphism $\partial_{\alpha}: M_{\alpha, \hat{Z}} \rightarrow M_{\alpha, \hat{Z}}$ of degree 1 which satisfies the product rule with respect to $s_{\alpha}: R_{\alpha, \widehat{Z}} \rightarrow R_{\alpha, \widehat{Z}}$, such that

$$
\partial_{\alpha}\left(m_{\beta, j}^{\alpha}\right)=\sum_{k=0}^{|\beta|-1}(-1)^{k} m_{\beta \backslash i_{k}, j}^{\alpha}
$$

in the case $|\beta|>0, a(\beta)=a(\alpha)$, and $\partial_{\alpha}\left(m_{\beta, j}^{\alpha}\right)=0$ otherwise. Obviously $\partial_{\alpha}^{2}=0$ and each $K_{\alpha}^{k}(\mathscr{F})_{\widehat{Z}}$ is stable under $\partial_{\alpha}$.

(6.7) Definition. A differential $s$ on $M_{\widehat{Z}}$ of degree 1 is called special if the following conditions are satisfied:

(1) $s$ fulfills the product rule (with respect to $\left.s: R_{\widehat{Z}} \rightarrow R_{\widehat{Z}}\right)$ and $\left(M_{\widehat{Z}}, s\right)$ is a resolution of $\Gamma\left(\bar{E}_{*, \widehat{Z}},\left(\varphi_{*}, \widehat{Z}\right)_{*}\left(\mathscr{F} \mid U_{*}, \hat{Z}\right)\right)$; moreover $s \mid M_{i, \widehat{Z}}=s_{i}$ for $i \in I$.

(2) $s$ leaves the filtration $K^{*}(\mathscr{F})_{\widehat{Z}}$ of $M_{\widehat{Z}}$ invariant and $\left(s-\partial_{\alpha}\right)\left(m_{\beta, j}^{\alpha}\right)$ is in $K_{\alpha}^{k+1}(\mathscr{F})_{\widehat{Z}}$ if $\psi\left(m_{\beta, j}^{\alpha}\right) \geq k \leq 0$.

(3) For $\alpha=(i, a) \in \mathscr{N}^{(1)}$, the restriction $s \mid M_{\alpha, \widehat{Z}}^{-1}$ coincides with the homomorphism in (6.3).

(4) If $\alpha=(i, a) \in \mathscr{N}^{(1)}$, then $s\left(m_{\alpha, j}^{\alpha}\right)=m_{a, j}^{\alpha}+h_{j}^{(\mathscr{F})}$ (see (6.5)) for $j \in$ $J_{a}^{-1}(\mathscr{F})$; observe that $h_{j}^{(\mathscr{F})}$ is in $K_{\alpha}^{0}(\mathscr{F})_{\widehat{Z}}$.

Again, we have the following assertion:

(6.8) Theorem. Let $S, X, \mathscr{F}, \mathscr{P}$, and $M$ be as in (6.1), (6.6). Then there exists a special differential $s$ on $M_{\widehat{Z}}$. (A resolution of the form $\left(M_{\widehat{Z}}, s\right)$ will be called special.)

The proof of [B-K, (IV. 1.7)] can be applied without difficulties to this situation. The differential $s$ on $M_{\alpha, \widehat{Z}}$ is constructed first by induction on $|\alpha|$ and then, also inductively, on each $K_{\alpha}^{k}(\mathscr{F})_{\widehat{Z}}$. 


\section{TANGENT COMPLEXES AND DESCRIPTION OF FORMAL SPACES} AND SHEAVES; SPLITTING THEOREM

(7.1) Let $T=(0, A)$ be a formal space germ, embedded in a smooth germ $\hat{Z}$ and $\mathscr{X}$ a space in $\operatorname{An}_{p}(T)$. Moreover, we fix a finite family of polyhedrons $\mathscr{P}=\left(P_{i}, \varphi_{i}, E_{i}\right)_{i \in I}$ for $\mathscr{X} / \widehat{Z}$ with nerve $\mathscr{N}$, satisfying the polyhedral axiom and $\left|\mathscr{P}^{\left(t_{0}\right)}\right|=|\mathscr{X}|$ for some $\left.t_{0} \in\right] 0,1[$ (see (5.4.1)), a free simplicial Calgebra $R=\Gamma\left(\bar{E}_{*}, \mathscr{R}\right)$, such that $R_{\widehat{Z}}$ has a differential $s$ with the property that $\left(R_{\widehat{Z}}, s\right)$ is a resolvent of $\Gamma\left(\bar{E}_{*, \widehat{Z}},\left(\varphi_{*}\right)_{*}\left(\mathscr{O}_{\mathscr{Z}} \mid U_{*}\right)\right)$. The fiber differential, induced by $s$, will be called $s_{0}: R \rightarrow R$.

From now on we consider $\mathscr{R}$ as a sheaf on the open simplicial polydisc $E_{*}$. We denote by ${ }^{\infty} L:=\operatorname{Der}(\mathscr{R}, \mathscr{R})$ the Z-graded $\mathbf{C}$-vector space, consisting of all (compatible) derivations of $\mathscr{R}$. For $m \in \mathbf{Z}$ we set $F^{m}:=F^{m}\left({ }^{\infty} L\right):=$ $\left\{u \in{ }^{\infty} L: u \mid \mathscr{R}^{j}=0\right.$ for $\left.j \geq-m\right\}$ and

$$
{ }^{m} L:=\operatorname{Der}(\mathscr{R}, \mathscr{R}) / F^{m}, \quad{ }^{m} \mathscr{L}:=\operatorname{Der}_{\widehat{Z}}\left(\mathscr{R}_{\widehat{Z}}, \mathscr{R}_{\widehat{z}}\right) / \mathscr{F}^{m},
$$

where $\mathscr{F}^{m}$ is defined analogously. Obviously, each homogeneous component of ${ }^{m} L$ is a PO-space by taking values of derivations on the free generators of $R$ and ${ }^{m} \mathscr{L}={ }^{m} L_{\mathscr{Q}_{\hat{\mathbf{z}}}}$ as graded objects. Moreover, ${ }^{m} L^{(\geq 0)}$ has the structure of a graded PO-Lie algebra over C (in the sense of [B-K, (II.9.5)]) and ${ }^{m} L^{0}$ is the Lie algebra of a PO-Lie group $G$ of type $(-1,1 ;-1)$. There is also an adjoint representation $\omega: G \times{ }^{m} L^{(\geq 0)} \rightarrow{ }^{m} L^{(\geq 0)}$, which is convergent of type $(-1,1 ;-1)$. The mapping

$$
\begin{aligned}
q:{ }^{m} L^{1} & \rightarrow{ }^{m} L^{2} \\
u & \mapsto \frac{1}{2}[u, u]
\end{aligned}
$$

is strictly convergent and equivariant with respect to $\omega$. By setting

$$
W_{\lambda}:=\left(\bar{q}_{\lambda}\right)^{-1}(0), \quad \lambda \in[1 / 2,1[,
$$

we get an inductive system $W_{\lambda}=\left(W_{\lambda}\right)_{\lambda}$ of banachanalytic spaces. The image of $s_{0}$ in the Banach space $\left({ }^{\bar{m} L^{1}}\right)_{\lambda}$ lies in $W_{\lambda}$ and is also denoted by $s_{0}$. From now on we always consider $W_{\lambda}$ as a banachanalytic space germ with distinguished point $s_{0}$. Using (4.2), (4.4), we see that the image of $s$ in ${ }^{m} \mathscr{L}^{-1}$ gives in a natural way a compatible system of formal maps

$$
s_{\lambda}: \widehat{Z} \rightarrow W_{\lambda}, \quad \lambda \in[1 / 2,1[.
$$

On $\operatorname{Der}_{\widehat{Z}}\left(\mathscr{R}_{\widehat{\mathrm{Z}}}, \mathscr{R}_{\widehat{\mathrm{Z}}}\right)$ we have the $\mathscr{O}_{\widehat{\mathrm{Z}}}$-linear differential $d(u):=[s, u]$, which projects down to ${ }^{m} \mathscr{L}$, such that $\left({ }^{m} \mathscr{L}, d\right)$ is a complex in $\mathrm{PO}_{1 / 2}\left(\mathscr{O}_{\hat{Z}}\right)$. On the special fiber over $0 \in \widehat{Z}$, we get that $d_{0}:{ }^{m} L \rightarrow{ }^{m} L$ is induced by $u \mapsto\left[s_{0}, u\right]$ on ${ }^{\infty} L$. 
(7.2) Let $n_{0}:=n(\mathscr{P})$ be as in (5.3) and $\nu \geq 1, m>n_{0}$ integers. We put (with the notation of $(2.8)$ )

$$
\bar{R}_{\widehat{Z}}:=R_{\widehat{Z}} / R_{\widehat{Z}}^{[\leq-(m+\nu+1)]}, \quad \tilde{R}_{\widehat{Z}}:=R_{\widehat{Z}}^{\left[\leq-n_{0}\right]} / R_{\widehat{Z}}^{[\leq-(m+\nu+1)]} .
$$

Then $\bar{R}_{\widehat{Z}}, \tilde{R}_{\widehat{Z}}$ are differential-graded modules over $R_{\widehat{Z}}$. By the choice of $n_{0}$, the complexes $R_{\alpha, \widehat{Z}}^{\left[\leq-n_{0}\right]}$ and $\tilde{R}_{\alpha, \widehat{Z}}$ are homotopic to zero for each $\alpha \in \mathscr{N}$ as complexes of free $R_{\alpha, \widehat{Z}^{0}}^{0}$-modules. Consequently, $\Gamma^{\prime}\left(E_{\alpha, \widehat{Z}}, \mathscr{R}_{\alpha, \widehat{Z}}^{\left[\leq-n_{0}\right]}\right)$ and $\Gamma^{\prime}\left(E_{\alpha, \widehat{Z}}, \tilde{\mathscr{R}}_{\alpha, \widehat{Z}}\right)^{6}$ are homotopic to zero as complexes in $\mathrm{PO}_{1 / 2}\left(\mathscr{O}_{\widehat{Z}}\right)$. If we define $\bar{R}$ and $\widetilde{R}$ analogously to (7.2.1), now with respect to $s_{0}$, we get on the special fiber

The graded objects

$$
\left(\bar{R}_{\widehat{Z}}\right)(0)=\bar{R}, \quad\left(\widetilde{R}_{\widehat{Z}}\right)(0)=\widetilde{R} .
$$

$$
\bar{L}:=\operatorname{Der}(\mathscr{R}, \overline{\mathscr{R}}), \quad \overline{\mathscr{L}}:=\operatorname{Der}_{\widehat{Z}}\left(\mathscr{R}_{\widehat{Z}}, \overline{\mathscr{R}}_{\widehat{Z}}\right),
$$

endowed with the differential $\bar{d}_{0}(u):=\bar{s}_{0} u-(-1)^{\operatorname{deg}(u)} u s_{0}$, resp. $\bar{d}(u):=\bar{s} u-$ $(-1)^{\operatorname{deg}(u)} u s$, are complexes in $\mathrm{PO}_{1 / 2}(\mathbf{C})$, resp. $\mathrm{PO}_{1 / 2}\left(\mathscr{O}_{\widehat{Z}}\right)$, with $\overline{\mathscr{L}}=\bar{L}_{\mathscr{O}_{\widehat{z}}}$ as graded objects. Moreover, there is a regular ${ }^{7}$ spectral sequence

$$
\operatorname{Tor}_{-p}^{\widehat{\widehat{Q}}}\left(\mathbf{C}, H^{q}(\overline{\mathscr{L}})\right) \Rightarrow H^{p+q}(\bar{L})
$$

and the cohomology modules

$$
H^{q}(\overline{\mathscr{L}})=T^{q}\left(\mathscr{X} / \widehat{Z}, \mathscr{O}_{\mathscr{L}}\right)
$$

are invariants of $\mathscr{X} / \widehat{Z}$ and so do not depend on $\mathscr{P}$ and $R$ (for a proof, compare for instance $\left[\mathrm{Pa}_{1}\right.$, p. 173]. One immediately gets that all restriction maps $\bar{L}_{t_{2}} \rightarrow \bar{L}_{t_{1}}$ are quasi-isomorphisms for $t_{0}<t_{1} \leq t_{2} \leq 1$, especially $\operatorname{dim}_{\mathrm{C}} H^{k}(\bar{L})<\infty$ for all $k$ by a standard argument.

Now we are going to make the analogous constructions in the module case.

(7.3) Let $S$ be a smooth complex space germ, $X$ a space in $\operatorname{An}(S)$ with separated structure map $X \rightarrow S$, and $T=(0, A)$ a formal space germ over $S$, embedded (over $S$ ) in $\hat{Z}$ with $Z=S \times Z^{\prime}$ and $Z^{\prime}$ smooth and convergent. Moreover, we fix a module $\mathscr{F}$ in $\operatorname{Coh}_{p}\left(X_{A}\right)$ and a finite family of polyhedrons $\mathscr{P}=\left(P_{i}, \varphi_{i}, E_{i}\right)_{i \in I}$ for $X / S$ with nerve $\mathscr{N}$, which satisfies the polyhedral axiom. We suppose that $\left|\mathscr{P}_{\widehat{Z}}^{\left(t_{0}\right)}\right| \supset \operatorname{supp}(\mathscr{F})$ holds for some $\left.t_{0} \in\right] 0,1[$ near by 1 .

Furthermore, we take a free simplicial graded C-algebra $R=\Gamma\left(\bar{E}_{*}, \mathscr{R}\right)$ with a differential $s_{X}$ on $R_{S}$, such that $\left(R_{S}, s_{X}\right)$ is a resolvent of the complex

$$
\Gamma\left(\bar{E}_{*, S},\left(\varphi_{*}\right)_{*}\left(\mathscr{O}_{X} \mid U_{*}\right)\right),
$$

\footnotetext{
${ }^{6}$ Here $\Gamma^{\prime}$ is the graded global section functor, i.e., $\Gamma^{\prime}\left(\amalg_{k} \mathscr{F}_{k}\right)=\amalg_{k} \Gamma\left(\mathscr{F}_{k}\right)$.

${ }^{7}$ In the sense of [EGA, $\left.(0.11 .1 .3)\right]$.
} 
and a free graded $R$-module $M$ (having only finitely many generators in each degree) with a differential $s$ on $M_{\widehat{Z}}$, compatible with $\left(s_{X}\right)_{\widehat{Z}}$, such that $\left(M_{\widehat{Z}}, s\right)$ is a resolution of $\Gamma\left(\bar{E}_{*}, \hat{Z},\left(\varphi_{*}, \hat{Z}\right)_{*}\left(\mathscr{F} \mid U_{*}, \hat{Z}\right)\right)$. From now on we consider $\mathscr{R}$ and $\mathscr{M}$ as (graded) sheaves on $E_{*}$. We put ${ }^{\infty} E:=\operatorname{Hom}_{\mathscr{R}}(\mathscr{M}, \mathscr{M})$ and, analogously to (7.1),

$$
{ }^{m} E:=\operatorname{Hom}_{\mathscr{R}}(\mathscr{M}, \mathscr{M}) / F^{m}, \quad{ }^{m} \mathscr{E}:=\operatorname{Hom}_{\mathscr{R}_{\widehat{Z}}}\left(\mathscr{M}_{\widehat{Z}}, \mathscr{M}_{\widehat{Z}}\right) / \mathscr{F}^{m}
$$

for $m \in \mathbf{Z}$. The homogeneous components of ${ }^{m} E$ are again PO-spaces (by taking values on the free generators of $M$ over $R$ ) and ${ }^{m} \mathscr{E}={ }^{m} E_{\mathscr{Q}_{\widehat{Z}}}$ as $\mathbf{Z}$ graded objects. Obviously, ${ }^{m} E^{(\geq 0)}$ has the structure of a graded PO-Lie algebra over C with strictly convergent Lie bracket [, ]: ${ }^{m} E^{(\geq 0)} \times{ }^{m} E^{(\geq 0)} \rightarrow{ }^{m} E^{(\geq 0)}$, induced by the usual graded commutator of homomorphisms. Again, ${ }^{m} E^{0}$ is the Lie algebra of a (strict) PO-Lie group $G$ and there is a strictly convergent adjoint representation $\omega: G \times{ }^{m} E^{(\geq 0)} \rightarrow{ }^{m} E^{(\geq 0)}$. On $\operatorname{Hom}_{\mathscr{R}_{\widehat{z}}}\left(\mathscr{M}_{\hat{z}}, \mathscr{M}_{\widehat{z}}\right)$ we have an $\mathscr{O}_{\widehat{Z}}$-linear differential $d$, given by $u \mapsto[s, u]$, which projects down to ${ }^{m} \mathscr{E}$, such that $\left({ }^{m} \mathscr{E}, d\right)$ is a complex in $\mathrm{PO}_{1 / 2}\left(\mathscr{O}_{\widehat{Z}}\right)$. Over the special fiber, this differential $d_{0}:=d(0)$ is induced by $u \mapsto\left[s_{0}, u\right]$, where $s_{0}: M \rightarrow M$ is the fiber differential associated to $s$.

Now let $\delta: \mathscr{M}_{S} \rightarrow \mathscr{M}_{S}$ be the $\mathscr{O}_{S}$-linear homomorphism with $\delta\left(m_{j}\right)=s_{0}\left(m_{j}\right)$ for any free generator $m_{j}$ of $M$ over $R$, such that $\delta$ fulfills the product rule with respect to $s_{X}$. Obviously $\delta^{2}=0$. If we define $w:=s-\delta_{\widehat{Z}}$, then $w$ is in $\operatorname{Hom}_{\mathscr{R}_{\widehat{Z}}}\left(\mathscr{M}_{\widehat{Z}}, \mathscr{M}_{\hat{Z}}\right)$ and $\left[\delta_{\widehat{Z}}, w\right]+\frac{1}{2}[w, w]=0$. The $S$-morphism $q:{ }^{m} E_{S}^{1} \rightarrow{ }^{m} E_{S}^{2}$, given (on general points) by the formula $q(u):=[\delta, u]+$ $\frac{1}{2}[u, u]$ is a strictly convergent morphism in $\operatorname{Con}(S)$, which is equivariant with respect to the (strictly convergent) operations $\omega_{S}^{1}[\delta]: G_{S} \times{ }_{S}^{m} E_{S}^{1} \rightarrow{ }^{m} E_{S}^{1}$ and $\omega_{S}^{2}: G_{S} \times{ }_{S}^{m} E_{S}^{2} \rightarrow{ }^{m} E_{S}^{2} \cdot{ }^{8}$ Setting

$$
W_{\lambda}:=\left(q_{\lambda}^{-1}(S \times\{0\}),\{0\} \times\{0\}\right), \quad \lambda \in[1 / 2,1[,
$$

then $W=\left(W_{\lambda}\right)_{\lambda}$ is an inductive system of banachanalytic space germs over $S$. Using the results of $\S 4$, the homomorphism $w$ induces a compatible system of formal $S$-maps

$$
w_{\lambda}: \widehat{Z} \rightarrow W_{\lambda}, \quad \lambda \in[1 / 2,1[.
$$

(7.4) We fix $m_{0}:=n_{0}+\operatorname{dim} Z^{\prime}$ and integers $\nu \geq 1, m>m_{0}$. Again, we define

$$
\begin{gathered}
\bar{M}_{\widehat{Z}}:=M_{\widehat{Z}} / M_{\widehat{Z}}^{[\leq-(m+\nu+1)]}, \\
\widetilde{M}_{\widehat{Z}}:=M_{\widehat{Z}}^{\left[\leq-m_{0}\right]} / M_{\widehat{Z}}^{[\leq-(m+\nu+1)]} .
\end{gathered}
$$

\footnotetext{
${ }^{8}$ Here $\omega_{S}^{1}[\delta]$ denotes the Taylor expansion of $\omega_{S}^{1}$ "around the point" $(0, \delta)$ which is well defined here!
} 
Then $M_{\widehat{Z}}, \widetilde{M}_{\widehat{Z}}$ are differential-graded modules over $R_{\widehat{Z}}$. As in (7.2), the complexes $\Gamma^{\prime}\left(E_{\alpha, \widehat{Z}}, \mathscr{M}_{\alpha, \widehat{Z}}^{\left[\leq-m_{0}\right]}\right)$ and $\Gamma^{\prime}\left(E_{\alpha, \widehat{Z}}, \widetilde{\mathscr{M}}_{\alpha, \widehat{Z}}\right)$ are homotopic to zero in $\mathrm{PO}_{1 / 2}\left(\mathscr{O}_{\hat{Z}}\right)$ and, with the obvious notation

$$
\left(\bar{M}_{\widehat{Z}}\right)(0)=\bar{M}, \quad\left(\widetilde{M}_{\widehat{Z}}\right)(0)=\widetilde{M}
$$

We put

$$
\bar{E}:=\operatorname{Hom}_{\mathscr{R}}(\mathscr{M}, \overline{\mathscr{M}}), \quad \overline{\mathscr{E}}:=\operatorname{Hom}_{\mathscr{R}_{\widehat{Z}}}\left(\mathscr{M}_{\widehat{Z}}, \overline{\mathscr{M}}_{\widehat{Z}}\right) .
$$

Then $\bar{E}$, resp. $\overline{\mathscr{E}}$, is a complex in $\mathrm{PO}_{1 / 2}(\mathbf{C})$, resp. $\mathrm{PO}_{1 / 2}\left(\mathscr{O}_{\widehat{Z}}\right)$, with $\overline{\mathscr{E}}=\bar{E}_{\mathscr{O}_{\widehat{Z}}}$ as graded objects. There is also a regular spectral sequence

$$
\operatorname{Tor}_{-p}^{\mathscr{Q}}\left(\mathbf{C}, H^{q}(\overline{\mathscr{E}})\right) \Rightarrow H^{p+q}(\bar{E})
$$

and

$$
H^{q}(\overline{\mathscr{E}})=\operatorname{Ext}_{X_{\widehat{Z}}}^{q}(\mathscr{F}, \mathscr{F})
$$

by standard arguments. Consequently, the restriction maps $\bar{E}_{t_{2}} \rightarrow \bar{E}_{t_{1}}$ are quasi-isomorphisms for $t_{0}<t_{1} \leq t_{2} \leq 1$ and $\operatorname{dim}_{\mathbf{C}} H^{k}(\bar{E})<\infty$ for all $k$.

(7.5) The construction in (7.4) can be immediately generalized to the following situation: Let $\mathscr{G}$ be a second module in $\operatorname{Coh}_{p}\left(X_{A}\right)$ with a resolution $\left(M_{\widehat{Z}}^{\prime}, s^{\prime}\right)$, such that the properties in (7.3) are fulfilled (where $\mathscr{P}, R$, and $t_{0}$ remain the same). Defining similarly

$$
\begin{aligned}
\bar{E} & :=\bar{E}(\mathscr{F}, \mathscr{G}):=\operatorname{Hom}_{\mathscr{R}}\left(\mathscr{M}, \overline{\mathscr{M}}^{\prime}\right), \\
\overline{\mathscr{E}} & :=\overline{\mathscr{E}}(\mathscr{F}, \mathscr{G}):=\operatorname{Hom}_{\mathscr{R}_{\widehat{Z}}}\left(\mathscr{M}_{\widehat{Z}}, \overline{\mathscr{M}}_{\widehat{Z}}^{\prime}\right),
\end{aligned}
$$

we have $\overline{\mathscr{E}}=\bar{E}_{\widehat{\mathscr{Z}}}$ (as graded objects of course) and $H^{q}(\overline{\mathscr{E}})=\operatorname{Ext}_{X_{\widehat{Z}}}^{q}(\mathscr{F}, \mathscr{G})$ (there is also a spectral sequence as in (7.4.2)).

For abbreviation, let " $K$ " be the common notation for " $L$ " and " $E$ " above. Then the following assertion holds (compare [B-K, (I.9.7)]):

(7.6) Proposition. (1) We have for $p \in\left[-\nu+1, m-m_{0}-2\right]$ :

$$
H^{p}\left(\left({ }^{m} K\right)^{\left(-\nu, m-m_{0}-1\right)}\right)=H^{q}\left({ }^{\infty} K\right) .
$$

(2) If homotopy maps $h_{\alpha}$ for $\widetilde{R}_{\alpha, \widehat{Z}}$ (resp. $\widetilde{M}_{\alpha, \widehat{Z}}^{\prime}$ ) are fixed for every $\alpha \in \mathscr{N}$ and, moreover, a splitting $r$ of $\bar{K}^{\left(-\nu, m-m_{0}\right)}$ exists in $P O$, then $\left(h_{\alpha}\right)_{\alpha}$ and $r$ define in a natural way a homotopy equivalence $u$ of $\bar{K}^{\left[-\nu+2, m-m_{0}-3\right]}$ onto $\left({ }^{m} K\right)^{\left[-\nu+2, m-m_{0}-3\right]}$ in the category of PO spaces.

The main result of this section is

(7.7) Theorem. The complex $\bar{K}_{t}$ splits in the category PO for any $\left.t \in\right] t_{0}, 1[$.

Together with (7.6) we obtain 
(7.8) Corollary. If $\nu \geq 3$ and $m \geq m_{0}+6$, then the complex

$$
\left({ }^{m} K^{0}\right)_{t} \stackrel{d_{0}}{\longrightarrow}\left({ }^{m} K^{1}\right)_{t} \stackrel{d_{0}}{\longrightarrow}\left({ }^{m} K^{2}\right)_{t}
$$

splits in PO for $t \in] t_{0}, 1[$.

Replacing eventually $\mathscr{P}$ by $\mathscr{P}^{(t)}$ for a $\left.t \in\right] t_{0}, 1[$, we may assume that the complex

$$
\left(\left({ }^{m} K\right)^{(0,2)}, d_{0}\right)
$$

splits in the category PO.

As the proof of (7.7) consists just of a translation of the corresponding result in [B-K] into the situation with formal basis $\hat{Z}$, we restrict ourselves here only to mentioning the formulation of the crucial steps in our context: By the absolute splitting criterion [B-K, (II.1.6)], it suffices to show that the restriction map $\bar{K} \rightarrow \bar{K}_{t}$ is

(a) a quasi-isomorphism,

(b) modulo homotopy, surjective in PO for $t \in] t_{0}, t_{1}[$.

Property (a) is clearly satisfied by (7.2), (7.4). For the proof of (b), one shows even more:

$(\mathrm{b})^{\prime} \overline{\mathscr{K}} \rightarrow \overline{\mathscr{K}}_{t}$ is, modulo homotopy, surjective in PO. $\left(\mathscr{O}_{\widehat{z}}\right)$, where we have replaced $\mathscr{L}$, resp. $\mathscr{E}$, by the symbol $\mathscr{K}$. The assertion (b) then follows, using the base change $\mathscr{O}_{\widehat{Z}} \rightarrow \mathscr{O}_{\widehat{Z}} / \mathfrak{m}_{\widehat{Z}}$. The main ingredients for verifying (b)' are the following:

(A) $\Omega_{R_{\widehat{Z}}}$ (resp. $M_{\widehat{Z}}$ ) is a homotopy invariant for different resolvents $R_{\widehat{Z}}$ (resp. different resolutions $M_{\widehat{Z}}$ );

(B) existence of admissible and (+)-admissible resolvents $R_{\widehat{Z}}, R_{\widehat{Z}}^{+}$; (resp. resolutions $M_{\widehat{Z}}, M_{\widehat{Z}}^{+}$) in the sense of [B-K, Kap. IV, $\left.\S \S 1,2\right]$;

(C) zero-homotopy of the (simplicial) Cech-complex of a resolution of a coherent sheaf on a polydisc (see [B-K, (II.5)]).

(7.9) Let $A$ be a formal algebra and $(\mathscr{K}, d)$ be a complex in $\mathrm{PO}_{\varepsilon}(A)$, resp. PO. $(A)$, such that the fiber complex $\left(\mathscr{K}(0), d_{0}\right)$ has a splitting $h$ in $\mathrm{PO}_{\varepsilon}(\mathbf{C})$, resp. PO. $(\mathbf{C})$. Without loss of generality, we may assume $h^{2}=0$ and $h d_{0} h=0$. Denote by $\mathscr{H}$ the complex $\left(H(\mathscr{K}(0))_{A}, 0\right)$. Since $\mathscr{K}=\mathscr{K}(0)_{A}$, the maps $\left(d_{0}\right)_{A}$ and $h_{A}$ are endomorphisms of $\mathscr{K}$, for which we write $d_{0}$, resp. $h$, again. Then $\operatorname{id}_{\mathscr{H}}-\left[d_{0}, h\right]$ induces homotopy equivalences

$$
f: \mathscr{H} \rightarrow\left(\mathscr{K}, d_{0}\right), \quad g:\left(\mathscr{K}, d_{0}\right) \rightarrow \mathscr{H}
$$

in $\mathrm{PO}_{\varepsilon}(A)$, resp. PO. $(A)$, with

$$
g f=\mathrm{id}_{\mathscr{H}}, \quad f g=\mathrm{id}_{\mathscr{H}}-\left[d_{0}, h\right] .
$$

If we set $r:=d-d_{0}$, then $\left(\operatorname{id}_{\mathscr{K}}+h r\right)$ is an endomorphism of $\mathscr{K}$, which is invertible in $\mathrm{PO}_{\varepsilon}(A)$, resp. PO. $(A)$. This follows from (4.9). Consequently

$$
\begin{gathered}
\partial:=g d\left(\mathrm{id}_{\mathscr{H}}+h r\right)^{-1} f: \mathscr{H} \rightarrow \mathscr{H}, \\
u:=\left(\operatorname{id}_{\mathscr{K}}+h r\right)^{-1} f: \mathscr{H} \rightarrow \mathscr{K}
\end{gathered}
$$


are well defined morphisms of degree 1 , resp. 0 , with $\partial(0)=0$, resp. $u(0)=$ $f$, such that $(\mathscr{H}, \partial)$ is a complex and $u:(\mathscr{H}, \partial) \rightarrow(\mathscr{K}, d)$ is a homotopy equivalence in $\mathrm{PO}_{\varepsilon}(A)$, resp. PO.(A) (see [B-K, (II.2.16)] for a proof). In particular, we have

(7.10) Theorem. Let $\mathscr{K}$ be a complex in $\mathrm{PO}_{\varepsilon}(A)$ with nuclear free components and $\left.t_{0} \in\right] 1-\varepsilon, 1[$, such that

(i) the "restriction" $\mathscr{K}(0) \rightarrow \mathscr{K}_{t_{0}}(0)$ is a quasi-isomorphism,

(ii) $\mathscr{K}_{t_{0}}(0)$ splits in $P O$.

Then there is a complex $\mathscr{H}$ in PO. $(A)$, whose homogeneous components are of the form $\mathscr{H}^{k}=V_{A}^{k}$ with $\operatorname{dim}_{\mathbf{C}} V^{k}<\infty$, and a homotopy equivalence $\mathscr{H} \rightarrow \mathscr{K}_{t_{0}}$ in PO. $(A)$.

\section{THE SURJECTIVITY OF THE FUNCTOR (1.4.1)}

(8.1) We take as a basis the situation of (7.1), (7.2) where we assume in addition that $\mathscr{P}$ is augmented and $\left(R_{\widehat{Z}}, s\right)$ is special. Moreover, we fix $m \geq$ $n(\mathscr{P})+6$ and $\left.\lambda_{.} \in\right] t_{0}, 1\left[\right.$. The morphism $s_{\lambda}$. from $(7.1 .1)$ can be restricted to $T \subset \widehat{Z}$ :

$$
\left(s_{\lambda_{0}}\right)_{T}: T \rightarrow W_{\lambda_{0}}
$$

Using (4.2), we obtain from this an $\mathscr{O}_{T}$-linear derivation $D\left(\left(s_{\lambda_{0}}\right)_{T}\right)$ on $\Gamma^{\prime}\left(E_{*, T}^{\left(\lambda_{*}\right)}, \mathscr{R}_{T}\right)$ of degree 1 which is trivial in degrees $<-m$ and with $D\left(\left(s_{\lambda_{*}}\right)_{T}\right)$ $=s_{T} \mid E_{*, T}^{\left(\lambda_{*}\right)}$ in degrees $\geq-m$. The situation in (7.1) gives us a system of data which fulfills the conditions in (3.2). More precisely, we put there $S=\operatorname{Spec}(C)$, $G$ the PO-Lie group with PO-Lie algebra ${ }^{m} L^{0}$ and $E:=\left({ }^{m} L\right)^{1}, F:=\left({ }^{m} L\right)^{2}$. Observe that $G$ operates on $F$ with fixed point $0 \in F$. Furthermore, the map $u:(E, 0) \rightarrow(F, 0)$ is by definition the Taylor expansion $q\left\langle s_{0}\right\rangle$ of $q$ around the point $s_{0} \in E$. Without loss of generality, we may assume that $\left({ }^{m} L\right)^{(0,2)}$ splits in $\mathrm{PO}_{1-t_{0}}$.

Then conditions (2) and (3) of Theorem (3.3) hold by (7.8). Condition (4) also holds by $(7.2),(7.6)(2)$. As the operation $\omega$ has all the desired properties in $(3.3)(1)$, we get from Proposition (3.3.2) that there exists a finite-dimensional subgerm $Y$ of $\left(W_{\lambda_{0}}-\left\{s_{0}\right\},\{0\}\right)$, such that

$$
\omega_{\lambda, \lambda_{\bullet}}\left[s_{0}\right]: G_{\lambda} \times Y \rightarrow W_{\lambda}-\left\{s_{0}\right\}
$$

is a smooth morphism of banachanalytic space germs for $t_{0}<\lambda<\lambda$. . In particular, we have a commutative diagram (with $Y^{\prime}=Y+\left\{s_{0}\right\}$ )

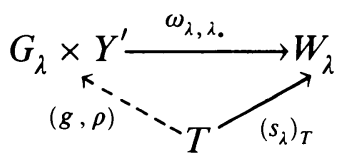


Now we take an exhaustion $A=\underline{\lim }_{k} A_{k}$ by convergent subalgebras $A_{k}$ of $A$ and call $T_{k}$ the associated space germ to $A_{k}$. Since $Y^{\prime}$ is finite dimensional, there is a certain $k$ and a morphism

$$
\rho_{k}: T_{k} \rightarrow Y^{\prime} \text { with } \rho=\left(\rho_{k}\right)_{T} .
$$

The composition $T_{k} \stackrel{\rho_{k}}{\longrightarrow} Y^{\prime} \stackrel{l}{\hookrightarrow} W_{\lambda}$. induces again an $A_{k}$-linear derivation $\delta$ on $\Gamma^{\prime}\left(E_{*, T_{k}}^{\left(\lambda_{*}\right)}, \mathscr{R}_{T_{k}}\right)$ of degree 1 such that $\delta=D\left(\imath \rho_{k}\right)$ and $\delta^{2}=0$. Trivially, we have $\delta(0)=s_{0}$ in degrees $\geq-m$ on the special fiber. We put

$$
\mathfrak{Y}_{*}:=\left(\dot{P}_{*}^{\left(\lambda_{*}\right)}, \mathscr{O}_{\mathfrak{Y}_{*}}\right) \text {, }
$$

where $\mathscr{O}_{\mathfrak{Y}_{\alpha}}:=\varphi_{\alpha}(0)^{-1}\left[\operatorname{Coker}\left(\mathscr{R}_{T_{k}}^{-1} \stackrel{\delta}{\rightarrow} \mathscr{R}_{T_{k}}^{0}\right) \mid E_{\alpha, T_{k}}^{\left(\lambda_{.}\right)}\right]$for $\alpha \in \mathscr{N}$. Then $\mathfrak{Y}_{*}$ is a simplicial complex space in $\mathbf{A n}\left(T_{k}\right)$. The morphism $g$ in (8.1.2) induces an isomorphism

$$
\mathfrak{Y}_{*, T}\left|\dot{P}_{*}^{(\lambda)} \stackrel{\sim}{\rightarrow} \mathscr{X}_{*}\right| \dot{P}_{*}^{(\lambda)}
$$

which is the identity in the distinguished fiber. According to the special choice of $\left(R_{\widehat{Z}}, s\right)$, we are able to show

(8.2) Theorem. $\mathfrak{Y}_{*}$ is the associate simplicial space to a complex space in $\operatorname{An}_{p}\left(T_{k}\right)$.

Proof. We put for abbreviation $S:=T_{k}$ and $\mathfrak{m}:=\mathfrak{m}_{S}$, the maximal ideal of $\mathscr{O}_{S}$. Moreover, we fix an arbitrary $\lambda$ in $] t_{0}, \lambda$. [. Obviously, it is sufficient to show: For each pair $\beta, \alpha \in \mathscr{N}^{(2)}$ with $\beta \subset \alpha$, the given $\mathscr{O}_{S}$-algebra homomorphism $\mathscr{O}_{\mathfrak{Y}_{\beta}} \rightarrow \mathscr{O}_{\mathfrak{Y}_{\alpha}}$ is an isomorphism in all points of $P_{\alpha}^{(\lambda)}$.

First we consider the case $\alpha=(i, a) \in \mathscr{N}^{(1)}$. By (5.11)(3), (5.8), resp. (5.11)(4), we have

$$
\begin{aligned}
& \delta\left(e_{\alpha, j}^{\alpha}\right) \equiv z_{j}^{a}-\psi_{j}^{(a, i)}(0) \bmod \mathfrak{m} \mathscr{R}_{\alpha, S}^{0}, \quad \text { for } j \in J_{a}^{0}, \\
& \delta\left(e_{\alpha, j}^{\alpha}\right) \equiv e_{a, j}^{\alpha}+h_{j}(0) \bmod \mathfrak{m} \mathscr{R}_{\alpha, S}^{-1}, \quad \text { for } j \in J_{a}^{-1},
\end{aligned}
$$

where $h_{j}$ is as in (5.9). We set

$$
\begin{gathered}
\mathscr{K}_{\alpha}^{0}:=\sum_{j \in J_{a}^{0}} \mathscr{R}_{\alpha, S}^{0} \delta\left(e_{\alpha, j}^{\alpha}\right), \\
\mathscr{K}_{\alpha}^{-1}:=\coprod_{j \in J_{a}^{0}} \mathscr{R}_{\alpha, S}^{0} e_{\alpha, j}^{\alpha}+\sum_{j \in J_{a}^{-1}} \mathscr{R}_{\alpha, S}^{0} \delta\left(e_{\alpha, j}^{\alpha}\right) .
\end{gathered}
$$

Then $\delta$ induces an exact sequence on $E_{\alpha, S}^{(\lambda)}$ :

$$
\mathscr{R}_{\alpha, S}^{-1} / \mathscr{K}_{\alpha}^{-1} \stackrel{\bar{\delta}_{\alpha}}{\longrightarrow} \mathscr{R}_{\alpha, S}^{0} / \mathscr{K}_{\alpha}^{0} \rightarrow\left(\varphi_{\alpha}(0)\right)_{*}\left(\mathscr{O}_{\mathfrak{Y}_{\alpha}}\right) \rightarrow 0 .
$$

(8.2.3) Lemma. (1) The canonical $\mathscr{O}_{S}$-algebra homomorphism

$$
\mathscr{R}_{i, S}^{0} \rightarrow \mathscr{R}_{\alpha, S}^{0} / \mathscr{K}_{\alpha}^{0}
$$


is an isomorphism on $\varphi_{\alpha}(0)\left(P_{\alpha}^{(\lambda)}\right)$ and the (on this set well-defined) composition

$$
\theta_{(i, a)}^{0}: \mathscr{R}_{a, S}^{0} \rightarrow \mathscr{R}_{\alpha, S}^{0} / \mathscr{K}_{\alpha}^{0} \stackrel{\sim}{\rightarrow} \mathscr{R}_{i, S}^{0}
$$

is, modulo $\mathfrak{m}$, induced by $z_{j}^{a} \mapsto \psi_{j}^{(a, i)}(0)$ for $j \in J_{a}^{0}$.

(2) The canonical module homomorphism (over $\theta_{(i, a)}^{0}$ )

$$
\mathscr{R}_{i, S}^{-1} \rightarrow \mathscr{R}_{\alpha, S}^{-1} / \mathscr{K}_{\alpha}^{-1}+\mathscr{K}_{\alpha}^{0} \mathscr{R}_{\alpha, S}^{-1}
$$

is an isomorphism on $\varphi_{\alpha}(0)\left(P_{\alpha}^{(\lambda)}\right)$ and the composition

$$
\theta_{(i, a)}^{-1}: \mathscr{R}_{a, S}^{-1} \rightarrow \mathscr{R}_{\alpha, S}^{-1} / \mathscr{K}_{\alpha}^{-1}+\mathscr{K}_{\alpha}^{0} \mathscr{R}_{\alpha, S}^{-1} \stackrel{\sim}{\rightarrow} \mathscr{R}_{i, S}^{-1}
$$

is, modulo $\mathfrak{m}$, given by $e_{a, j} \mapsto \sum_{j^{\prime} \in J_{i}^{-1}} h_{j, j^{\prime}}^{(a)}(0) e_{i, j^{\prime}}$ for $j \in J_{a}^{-1}$.

Proof. Follows from (8.3), (8.4) together with the formulas (8.2.1), (8.2.2).

Using (5.6.3), (5.6.5), we get that $\theta_{(i, a)}^{0}, \theta_{(i, a)}^{-1}$ are isomorphisms (observe that $J_{a}^{\nu}=J_{i}^{\nu}$ for $\left.\nu \in\{0,-1\}\right)$. Consequently, in the commutative diagram, given on $P_{\alpha}^{(\lambda)}$

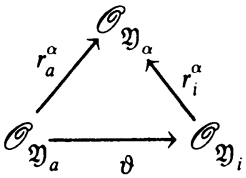

where $\vartheta$ is induced by $\theta_{(i, a)}^{0}$, all morphisms are isomorphisms.

Next, we consider the case $\alpha=\left(i_{0}, i_{1}, a\right) \in \mathscr{N}^{(2)}$. Because of the commutative diagram (on $P_{\alpha}^{(\lambda)}$ )

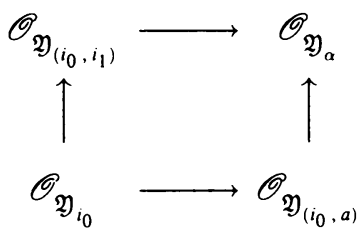

it obviously suffices to treat the case $\beta=\left(i_{0}, i_{1}\right)$. By construction of $R$, we can write

$$
\begin{aligned}
\mathscr{R}_{\alpha, S}^{-1}= & \left(r_{\beta}^{\alpha}\right)^{*}\left(\mathscr{R}_{\beta, S}^{-1}\right) \amalg \coprod_{j \in J_{a}^{0}} \mathscr{R}_{\alpha, S}^{0} e_{\left(i_{0}, a\right), j}^{\alpha} \\
& \amalg \coprod_{j \in J_{a}^{0}} \mathscr{R}_{\alpha, S}^{0} e_{\left(i_{1}, a\right), j}^{\alpha} \amalg \coprod_{j \in J_{a}^{-1}} \mathscr{R}_{\alpha, S}^{0} e_{a, j}^{\alpha} .
\end{aligned}
$$

Modulo $\mathfrak{m} \mathscr{R}_{\alpha, S}$, we get

$$
\begin{gathered}
\delta\left(e_{\left.\left(i_{0}, a\right), j\right)}^{\alpha}\right) \equiv e_{a, j}^{\alpha}+h_{j}(0), \quad \text { for } j \in J_{a}^{-1}, \\
\delta\left(e_{\alpha, j}^{\alpha}\right) \equiv e_{\left(i_{1}, a\right), j}^{\alpha}-e_{\left(i_{0}, a\right), j}^{\alpha}+v_{j}^{\alpha}\left(z^{i_{0}}, z^{i_{1}}\right), \quad \text { for } j \in J_{\alpha}^{0}
\end{gathered}
$$


(for the last congruence see $(5.11)(2)$ in the case $k=0$ ). This time we define

$$
\begin{gathered}
\mathscr{K}_{\alpha}^{0}:=\sum_{j \in J_{a}^{0}} \mathscr{R}_{\alpha, S}^{0} \delta\left(e_{\left(i_{0}, a\right), j}^{\alpha}\right), \\
\mathscr{K}_{\alpha}^{-1}:=\coprod_{j \in J_{a}^{0}} \mathscr{R}_{\alpha, S}^{0} e_{\left(i_{0}, a\right), j}^{\alpha}+\sum_{j \in J_{a}^{0}} \mathscr{R}_{\alpha, S}^{0} \delta\left(e_{\alpha, j}^{\alpha}\right)+\sum_{j \in J_{a}^{-1}} \mathscr{R}_{\alpha, S}^{0} \delta\left(e_{\left(i_{0}, a\right), j}^{\alpha}\right),
\end{gathered}
$$

and get the short exact sequence (on $\left.E_{\alpha, S}^{\left(\lambda_{0}\right)}\right)$ :

$$
\mathscr{R}_{\alpha, S}^{-1} / \mathscr{K}_{\alpha}^{-1} \stackrel{\bar{\delta}_{\alpha}}{\longrightarrow} \mathscr{R}_{\alpha, S}^{0} / \mathscr{K}_{\alpha, S}^{0} \rightarrow\left(\varphi_{\alpha}(0)\right)_{*}\left(\mathscr{O}_{\mathfrak{Y}_{\alpha}}\right) \rightarrow 0 \text {. }
$$

Similarly to $(8.2 .3)$, we have

(8.2.6) Lemma. The canonical morphisms

$$
\mathscr{R}_{\beta, S}^{0} \rightarrow \mathscr{R}_{\alpha, S}^{0} / \mathscr{K}_{\alpha, S}^{0}, \quad \mathscr{R}_{\beta, S}^{-1} \rightarrow \mathscr{R}_{\alpha, S}^{-1} / \mathscr{K}_{\alpha, S}^{-1}+\mathscr{K}_{\alpha, S}^{0} \mathscr{R}_{\alpha, S}^{-1}
$$

are isomorphisms on $\varphi_{\alpha}(0)\left(P_{\alpha}^{(\lambda)}\right)$.

Proof. Follows from the formulas (8.2.1)-(8.2.5) and (8.3), (8.4).

The following two easy lemmas were used above.

(8.3) Lemma. Let $A \rightarrow B$ be a local homomorphism of noetherian local rings and $M=\coprod_{j \in J} B e_{j}$ a finite free $B$-module. Moreover, let $f_{j} \in M, j \in J^{\prime} \subset J$, be elements, such that there is an invertible matrix $G=\left(G_{j, j^{\prime}}\right)$ in $\mathrm{GL}\left(\left|J^{\prime}\right|, B\right)$ with $f_{j} \equiv \sum_{j^{\prime}} G_{j, j^{\prime}} e_{j^{\prime}} \bmod \sum_{j \in J^{\prime} \backslash J} B e_{j}+\mathfrak{m}_{A} M$.

Then the inclusion $\coprod_{j \in J \backslash J^{\prime}} B e_{j} \hookrightarrow M$ induces an isomorphism

$$
\coprod_{j \in J \backslash J^{\prime}} B e_{j} \rightarrow M / \sum_{j \in J^{\prime}} B f_{j}
$$

of $B$-modules.

(8.4) Lemma. Let $A$ be an analytic algebra and $B=A\{z, w\}$ a smooth analytic A-algebra with variables $z=\left(z_{1}, \ldots, z_{n}\right), w=\left(w_{1}, \ldots, w_{m}\right)$. Moreover, let $g_{j} \in \mathfrak{m}_{B}$ be elements for $1 \leq j \leq n$, such that

$$
g_{j} \equiv z_{j}+\psi_{j}(w) \bmod \mathfrak{m}_{A} B .
$$

Then the canonical A-algebra homomorphism

$$
A\{w\} \rightarrow B / \sum_{j} g_{j} B
$$

is an isomorphism.

\section{THE SURJECTIVITY OF THE FUNCTOR (1.1.2)}

(9.1) In this section we take as a basis the situation of (7.3), where we assume in addition that $\mathscr{P}$ is augmented for $(X / S ; \mathscr{F})$ and $\left(M_{\widehat{Z}}, s\right)$ is a special 
resolution of $\mathscr{F} \mid \mathscr{P}_{\widehat{Z}}$ (in the sense of $\S 6$ ). Moreover, we fix integers $m_{0}:=$ $n(\mathscr{P})+\operatorname{dim} Z^{\prime}, \quad m \geq m_{0}+6$ and a number $\left.\lambda . \in\right] t_{0}, 1[$. Restricting the $S$-morphism $w_{\lambda_{0}}: \hat{Z} \rightarrow W_{\lambda}$ in (7.3.1) to the subspace $T \subset \widehat{Z}$, we obtain

$$
\left(w_{\lambda_{0}}\right)_{T}: T \rightarrow W_{\lambda_{\bullet}} \text {. }
$$

Using the results of $\S 4$, this morphism induces in a natural way an $\mathscr{O}_{T}$-linear differential $D\left(\left(w_{\lambda_{0}}\right)_{T}\right)$ of degree 1 on $\Gamma^{\prime}\left(E_{*, T}^{\left(\lambda_{*}\right)}, \mathscr{M}_{T}\right)$ which satisfies the product rule with respect to $s_{X_{T}}$ and such that $D\left(\left(w_{\lambda_{0}}\right)_{T}\right)(0)=s_{0}$ in degrees $\geq-m$.

Again, we can construct a system of data as in (3.2): This time we set, with the same $S, E:=\left({ }^{m} E\right)^{1}, F:=\left({ }^{m} E\right)^{2}$, and $G:=S \times G_{0}$, where $G_{0}$ is the PO-Lie group with PO-Lie algebra $\left({ }^{m} E\right)^{0}$ (which has strictly convergent multiplication and inversion in this case). For $\sigma: S \rightarrow\left(F_{S}, 0\right)$, we take the zero-section and $u:\left(E_{S}, 0\right) \rightarrow\left(F_{S}, 0\right)$ is by definition the morphism $q$, specified in (7.3). Without loss of generality, we may assume that the complex $\left(\left({ }^{m} E\right)^{(0,2)}, d_{0}\right)$ splits in the category $\mathrm{PO}_{1-t_{0}}($ see (7.8)). Now, since the tangent map of the orbit morphism $\beta: G_{0} \rightarrow(E, 0)$, associated to $\omega^{1}\left[s_{0}\right]: G_{0} \times(E, 0) \rightarrow(E, 0)$, identifies with $-d_{0}:\left({ }^{m} E\right)^{0} \rightarrow\left({ }^{m} E\right)^{1}$ and $T\left(u_{0}\right)=d_{0}:\left({ }^{m} E\right)^{1} \rightarrow\left({ }^{m} E\right)^{2}$, the conditions (1) $-(3)$ in (3.3) are obviously fulfilled. The finiteness condition (4) is clearly satisfied, according to (7.4), (7.6). From (the proof of) (3.3.2), we get that there is a finite-dimensional subgerm $Y$ of $W_{\lambda_{\text {o }}}$, such that

$$
\left(\omega_{\lambda, \lambda_{0}}^{1}\right)_{S}[\delta]: G_{\lambda} \times{ }_{S} Y \rightarrow W_{\lambda}
$$

is a smooth morphism of banachanalytic space germs over $S$ for $t_{0}<\lambda<\lambda$. Consequently, we have a commutative diagram of banachanalytic space germs over $S$

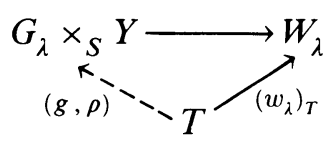

for each such $\lambda$. Now, we fix an exhaustion $A=\underline{\lim }_{k} A_{k}$ as in (1.1.1). It follows again that there is a $S$-morphism $\rho_{k}: T_{k} \rightarrow Y$ for some $k$ with $\rho=$ $\left(\rho_{k}\right)_{T}$. Here $T_{k}$ is the associated space germ to $A_{k}$. If $l: Y \hookrightarrow W_{\lambda}$. denotes the inclusion, we get an $A_{k}$-linear differential $D=D\left(\iota \rho_{k}\right)$ of degree 1 on $\Gamma^{\prime}\left(E_{*, T_{k}}^{\left(\lambda_{*}\right)}, \mathscr{M}_{T_{k}}\right)$, compatible with $s_{X_{T_{k}}}$ and $D(0)=s_{0}$ in degrees $\geq-m$. We set

$$
\mathscr{G}_{*}:=\left(\dot{\varphi}_{*, T_{k}}^{\left(\lambda_{*}\right)}\right)^{*}\left[\operatorname{Coker}\left(\mathscr{M}_{T_{k}}^{-1} \stackrel{D}{\rightarrow} \mathscr{M}_{T_{k}}^{0}\right)\right] .^{9}
$$

Then $\mathscr{G}_{*}$ is a coherent simplicial sheaf on $\left(\dot{P}_{*, T_{k}}^{\left(\lambda_{0}\right)}\right)$. The commutativity of (9.1.2) shows that the $S$-morphism $g: T \rightarrow G_{\lambda}$ induces an isomorphism

$$
\mathscr{G}_{*, T}\left|\dot{P}_{*, T}^{(\lambda)} \stackrel{\sim}{\rightarrow} \mathscr{F}_{*}\right| \dot{P}_{*, T}^{(\lambda)}
$$

\footnotetext{
${ }^{9}$ Here $\dot{\varphi}_{*}$ denotes the embedding $\dot{P}_{*} \hookrightarrow E_{*, S}$, induced by $\varphi_{*}$.
} 
which is the identity on the special fiber. Since $\left(M_{\widehat{Z}}, s\right)$ is special, we are able to prove

(9.2) Theorem. $\mathscr{G}_{*}$ is the associated simplicial sheaf to a sheaf in $\mathbf{C o h}_{p}\left(X_{T_{k}}\right)$. Proof. For abbreviation, we put $V:=T_{k}, \mathfrak{m}:=\mathfrak{m}_{V}$, and $\mathscr{N}:=\operatorname{Ner}(\mathscr{P})$. Obviously, it is sufficient to show: For any $\beta, \alpha \in \mathscr{N}^{(2)}$ with $\beta \subset \alpha$, the canonical homomorphism $G_{\beta} \rightarrow G_{\alpha}$ is an isomorphism on $P_{\alpha, V}^{(\lambda)}$ for each $\lambda \in$ ]$t_{0}, \lambda .[$.

First we consider the case $\alpha=(i, a) \in \mathscr{N}^{(1)}$. By (6.7)(3), (4), (6.6), we have modulo $\mathfrak{m} \mathscr{M}_{\alpha, V}$ :

$$
\begin{gathered}
D\left(m_{\alpha, j}^{\alpha}\right) \equiv m_{a, j}^{\alpha}-\Phi_{(i, a)}^{(0)}\left(m_{a, j}\right)(0), \quad \text { for } j \in J^{0}(\mathscr{F}), \\
D\left(m_{\alpha, j}^{\alpha}\right) \equiv m_{a, j}^{\alpha}+h_{j}^{(\mathscr{F})}(0), \quad \text { for } j \in J^{-1}(\mathscr{F}) .
\end{gathered}
$$

We set

$$
\mathscr{Q}:=\mathscr{M}_{S} / \mathscr{R}_{S}^{[\leq 0]} \mathscr{M}_{S},
$$

where $\mathscr{R}_{S}^{[\leq 0]}$ denotes the truncation ideal with respect to the differential $s_{X}$. The homogeneous components of $\mathscr{Q}_{\alpha}$ are free modules over $\mathscr{O}_{\alpha}:=\varphi_{\alpha *}^{(\lambda)}\left(\mathscr{O}_{X} \mid P_{\alpha}^{(\lambda)}\right)$ for every $\alpha$. Obviously, $D$ induces a homomorphism

$$
\bar{D}: \mathscr{O}_{V} \rightarrow \mathscr{O}_{V}
$$

of degree 1 . This time we define

$$
\begin{gathered}
\mathscr{K}_{\alpha}^{0}:=\sum_{j \in J^{0}(\mathscr{F})} \mathscr{O}_{\alpha, V} \bar{D}\left(m_{\alpha, j}^{\alpha}\right), \\
\mathscr{K}_{\alpha}^{-1}:=\coprod_{j \in J^{0}(\mathscr{F})} \mathscr{O}_{\alpha, V} m_{\alpha, j}^{\alpha}+\sum_{j \in J^{-1}(\mathscr{F})} \mathscr{O}_{\alpha, V} \bar{D}\left(m_{\alpha, j}^{\alpha}\right)
\end{gathered}
$$

and get the exact sequence on $\bar{E}_{\alpha, V}^{(\lambda)}$ :

$$
\mathscr{Q}_{\alpha, V}^{-1} / \mathscr{K}_{\alpha}^{-1} \stackrel{\bar{D}_{\alpha}}{\rightarrow} \mathscr{Q}_{\alpha, V}^{0} / \mathscr{K}_{\alpha, V}^{0} \rightarrow\left(\varphi_{\alpha, V}\right)_{*}\left(\mathscr{G}_{\alpha}\right) \rightarrow 0 .
$$

Similarly to (8.2.3), the following assertion holds.

(9.2.3) Lemma. On $\varphi_{\alpha, V}\left(P_{\alpha, V}^{(\lambda)}\right)$ we have

(1) the canonical homomorphisms

$$
\mathscr{Q}_{i, V}^{\nu} \rightarrow \mathscr{Q}_{\alpha, V}^{\nu} / \mathscr{K}_{\alpha}^{\nu}
$$

are isomorphisms for $\nu=0,-1$,

(2) modulo $\mathfrak{m}$, the composition

$$
\mathscr{Q}_{a, V}^{\nu} \rightarrow \mathscr{Q}_{\alpha, V}^{\nu} / \mathscr{K}_{\alpha}^{\nu} \stackrel{\sim}{\rightarrow} \mathscr{Q}_{i, V}^{\nu}
$$


is induced by

$$
\begin{gathered}
m_{a, j} \mapsto \Phi_{\alpha}^{(0)}\left(m_{\alpha, j}\right)(0), \quad \text { for } \nu=0, \\
m_{a, j} \mapsto \sum_{j^{\prime}} h_{j, j^{\prime}}^{(\mathscr{F})}(0) m_{i, j^{\prime}}, \quad \text { for } \nu=-1
\end{gathered}
$$

(compare (6.14), resp. (6.5), (6.7)(4)).

From (9.2.3), (6.1.4) we immediately get that in the commutative diagram

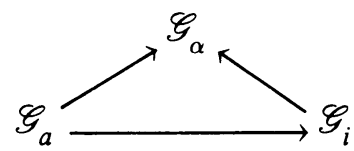

all arrows are isomorphisms.

It remains to consider the case where $\alpha=\left(i_{0}, i_{1}, a\right)$ is in $\mathscr{N}^{(2)}$. By the same argument as in $\S 8$, we may restrict to the case $\beta=\left(i_{0}, i_{1}\right)$. Here we put

$$
\begin{aligned}
\mathscr{K}_{\alpha}^{0}:= & \sum_{j \in J^{0}(\mathscr{F})} \mathscr{O}_{\alpha, V} \bar{D}\left(m_{\left(i_{0}, a\right), j}^{\alpha}\right), \\
\mathscr{K}_{\alpha}^{-1}:= & \coprod_{j \in J^{0}(\mathscr{F})} \mathscr{O}_{\alpha, V} m_{\left(i_{0}, a\right), j}^{\alpha}+\sum_{j \in J^{0}(\mathscr{F})} \mathscr{O}_{\alpha, V} \bar{D}\left(m_{a, j}^{\alpha}\right) \\
& +\sum_{j \in J^{-1}(\mathscr{F})} \mathscr{\mathscr { O }}_{\alpha, V} \bar{D}\left(m_{\left(i_{0}, a\right), j}^{\alpha}\right),
\end{aligned}
$$

and get the short exact sequence on $\bar{E}_{\alpha, V}^{(\lambda)}$

$$
\mathscr{Q}_{\alpha, V}^{-1} / \mathscr{K}_{\alpha}^{-1} \stackrel{\bar{D}_{\alpha}}{\longrightarrow} \mathscr{Q}_{\alpha, V}^{0} / \mathscr{K}_{\alpha}^{0} \rightarrow\left(\varphi_{\alpha, V}\right)_{*}\left(\mathscr{G}_{\alpha}\right) \rightarrow 0 .
$$

(9.2.4) Lemma. The canonical homomorphisms

$$
\mathscr{Q}_{\beta, V}^{\nu} \rightarrow \mathscr{Q}_{\alpha, V}^{\nu} / \mathscr{K}_{\alpha}^{\nu}
$$

are isomorphisms on $\varphi_{\alpha, V}\left(P_{\alpha, V}^{(\lambda)}\right)$ for $\nu=0,-1$.

Proof. Follows from (8.3) by using the formulas (9.2.1), (9.2.2), and (6.7)(2).

As it is not a restriction to assume $S$ to be smooth in (1.1.2), the surjectivity of this functor is established.

\section{The FUlly faithfulNess}

We first consider the case of modules.

(10.1) Let the situation be as in (1.1) and $\mathscr{F}, \mathscr{G}$ two modules in $\operatorname{Coh}_{p}\left(X_{A_{i_{0}}}\right)$ for some $i_{0} \in I$. We have to show that the natural map

$$
\left.\underset{i \geq i_{0}}{\lim _{X_{A_{i}}}} \operatorname{Hom}_{A_{i}}, \mathscr{G}_{A_{i}}\right) \rightarrow \operatorname{Hom}_{X_{A}}\left(\mathscr{F}_{A}, \mathscr{G}_{A}\right)
$$

is bijective. This is achieved in the following way: 
"Surjectivity $\Rightarrow$ Injectivity." Let $\varphi: \mathscr{F}_{A_{i}} \rightarrow \mathscr{G}_{A_{i}}$ be a homomorphism with $\varphi_{A}=0$. If we denote by $\mathscr{H}$ the cokernel of $\varphi$ and by $\psi: \mathscr{G}_{A_{i}} \rightarrow \mathscr{H}$ the quotient map, then $\psi_{A}$ is an isomorphism. So there is a map $\chi: \mathscr{H}_{A_{j}} \rightarrow \mathscr{G}_{A_{j}}$ for some $j \geq i$ with $\chi_{A}=\left(\psi_{A}\right)^{-1}$. Obviously, $\chi$ is an epimorphism (Nakayamalemma) and $\chi \psi_{A_{j}}$ is too. But this last fact implies that $\psi_{A_{j}}$ itself is an isomorphism and consequently $\varphi_{A_{j}}=0$.

Surjectivity. It is no restriction to assume $S$ to be smooth and $\mathscr{F}, \mathscr{G}$ in $\operatorname{Coh}_{p}(X)$. Let $T$, resp. $T_{i}$, be the associated space germ to $A$, resp. $A_{i}$. We fix an $S$-embedding $T \rightarrow \widehat{Z}$ as in (7.3) and, moreover, a finite family of polyhedrons $\mathscr{P}$ for $X / S$, satisfying the polyhedral axiom, with

$$
\left|\mathscr{P}^{\left(t_{0}\right)}\right| \supset \operatorname{supp}(\mathscr{F}) \cup \operatorname{supp}(\mathscr{G})
$$

for some $t_{0} \in$ ]0,1[ near by 1 . Let $R, s_{X}$ be as in (7.3) and $M$ a free (graded) $R$-module with the property that $M_{Z}$ has a differential $s$, compatible with $s_{X_{Z}}$, and such that $\left(M_{Z}, s\right)$ is a resolution of $\Gamma\left(\bar{E}_{*, Z},\left(\varphi_{*, Z}\right)_{*}\left(\mathscr{F}_{Z} \mid P_{*, Z}\right)\right)$.

Next, we take a free $R$-module $M^{\prime}$ and a differential $s^{\prime}$ on $M_{\widehat{Z}}^{\prime}$, compatible with $s_{X_{\widehat{Z}}}$, such that $\left(M_{\widehat{\mathrm{Z}}}^{\prime}, s^{\prime}\right)$ is a resolution of $\Gamma\left(\bar{E}_{*, \widehat{\mathrm{Z}}},\left(\varphi_{*}, \widehat{\mathrm{Z}}\right)_{*}\left(\mathscr{G}_{T} \mid P_{*, \widehat{\mathrm{Z}}}\right)\right)$ and $s_{T}^{\prime}: M_{T}^{\prime-1} \rightarrow M_{T}^{\prime 0}$ is already defined over $S$. We consider the complex

$$
\overline{\mathscr{E}}:=\left(\operatorname{Hom}_{\mathscr{R}_{\widehat{Z}}}\left(\mathscr{M}_{\widehat{Z}}, \overline{\mathscr{M}}^{\prime}{ }_{\hat{Z}}\right), d\right)
$$

constructed in (7.4), (7.5). Similarly to (7.7), the fiber complex $\overline{\mathscr{E}}_{t}(0)$ splits in PO.(C) for any $t \in] t_{0}, 1$ [. After shrinking, it is possible to assume the splitting for $\left(\overline{\mathscr{E}}(0), d_{0}\right)$. We have

$$
H^{0}(\overline{\mathscr{E}})=\operatorname{Hom}_{X_{\widehat{Z}}}\left(\mathscr{F}_{\widehat{Z}}, \mathscr{G}_{T}\right)=\operatorname{Hom}_{X_{T}}\left(\mathscr{F}_{T}, \mathscr{G}_{T}\right) .
$$

Now, take an element $\varphi$ in $\operatorname{Hom}_{X_{T}}\left(\mathscr{F}_{T}, \mathscr{G}_{T}\right)$ and consider it a zero-cycle in $\overline{\mathscr{E}}_{T}$. We adopt the notation from (7.9) with $\mathscr{K}:=\overline{\mathscr{E}}_{T}$ and $h$ a splitting of $\mathscr{K}(0)$. We may assume that $\varphi=u(\psi)$ with a zero-cycle $\psi$ in $(\mathscr{H}, \partial)$. Since $\mathscr{H}$ is a complex with finite free components, there is a zero-cycle $\psi^{\prime}$ in some $\left(\mathscr{K}_{T_{i}}, \partial_{T_{i}}\right)$ with $\psi_{T}^{\prime}=\psi$. It is now sufficient to show that $(u(\psi)) \mid \mathscr{M}_{T}^{(\geq-1)}$ is already defined over $T_{i}$ (observe that $s_{T}^{\prime} \mid \mathscr{M}_{T}^{\prime(\geq-1)}$ is defined over $S$ ). By formula (7.9.2) and the convergent expansion $\left(\mathrm{id}_{\mathscr{K}}+h r\right)^{-1}=\mathrm{id}_{\mathscr{K}}-(h r)+$ $(h r)^{2}-\cdots$, this follows from the

Assertion. Let $\eta$ be in $\mathscr{K}^{0}$ with $\eta \mid \mathscr{K}_{T}^{(\geq-1)}$ defined over $T_{i}$. Then

$$
(h r)(\eta) \mid \mathscr{K}_{T}^{(\geq-1)}
$$

is also defined over $T_{i}$.

Proof. By definition of $h$ and $r$, it suffices to see that $d_{T}(\eta) \mid \mathscr{M}_{T}^{(\geq-1)}$ is defined over $T_{i}$. But this is clear from the formula $d_{T}(\eta)=\overline{s_{T}^{\prime}} \eta-\eta s_{T}$, and because $s_{T}$ and $\bar{s}_{T}^{\prime} \mid \mathscr{M}_{T}^{\prime(\geq-1)}$ are even defined over $S$. 
(10.2) Next we treat the case of spaces, i.e., the fully faithfulness of the functor (1.4.1). For this let $X, Y$ be spaces in $\operatorname{An}_{p}\left(A_{i_{0}}\right)$ for some $i_{0} \in I$. We have to show the bijectivity of the map

$$
{\underset{i \geq i m}{i \geq i_{0}}}_{\operatorname{Hom}_{A_{i}}}\left(X_{A_{i}}, Y_{A_{i}}\right) \rightarrow \operatorname{Hom}_{A}\left(X_{A}, Y_{A}\right) \text {. }
$$

Surjectivity. Let $\varphi: X_{A} \rightarrow Y_{A}$ be a morphism in $\operatorname{An}(A)$ and $\Gamma_{\varphi} \subset$ $\left(X_{A} \times{ }_{A} Y_{A}\right)=\left(X \times \times_{A_{i_{0}}} Y\right)_{A}$ the graph of $\varphi$. By the surjectivity of (1.1.2), there is an index $i \geq i_{0}$ and a closed subspace $\Gamma \subset X_{A_{i}} \times_{A_{i}} Y_{A_{i}}$ with $\Gamma_{A}=\Gamma_{\varphi}$. If we consider now the projection $\mathrm{pr}: \Gamma \rightarrow X_{A_{i}}$, then $\mathrm{pr}_{A}$ is an isomorphism and, in particular, pr is an embedding. The fully faithfulness of (1.1.2) implies that $(\mathrm{pr})_{A_{j}}$ is an isomorphism for some $j \geq i$, so we are done.

Injectivity. Let $\varphi, \psi$ be in $\operatorname{Hom}_{A_{i}}\left(X_{A_{i}}, Y_{A_{i}}\right)$ with $\varphi_{A}=\psi_{A}$. If we denote by $\Gamma_{\varphi}$, resp. $\Gamma_{\psi}$, the graph of $\varphi$, resp. $\psi$, considered as a subspace of $X_{A_{i}} \times_{A_{i}} Y_{A_{i}}$, we have $\left(\Gamma_{\varphi}\right)_{A}=\Gamma_{\varphi_{A}}=\Gamma_{\psi_{A}}=\left(\Gamma_{\psi}\right)_{A}$. The subspace $\left(\Gamma_{\varphi} \cap \Gamma_{\psi}\right) \hookrightarrow \Gamma_{\varphi}$ of $\Gamma_{\varphi}$ has the property $\left(\Gamma_{\varphi} \cap \Gamma_{\psi}\right)_{A}=\left(\Gamma_{\varphi}\right)_{A}$ and so, by the same argument as above, we get $\left(\Gamma_{\varphi} \cap \Gamma_{\psi}\right)_{A_{j}}=\left(\Gamma_{\varphi}\right)_{A_{j}}$ for some $j \geq i$ or, alternatively, $\Gamma_{\varphi_{A_{j}}} \cap \Gamma_{\psi_{A_{j}}}=\Gamma_{\varphi_{A_{j}}}$. This proves $\Gamma_{\varphi_{A_{j}}} \subset \Gamma_{\psi_{A_{j}}}$. By symmetry, we may assume $\Gamma_{\varphi_{A_{j}}}=\Gamma_{\psi_{A_{j}}}$, which shows the injectivity.

\section{Proof of the local Statements in $\S 1$}

We first show Theorem (1.9). Since the proof is partly very similar to that of $\S 9$, we shall be brief at some points. By $T$, resp. $T_{i}$, resp. $T^{(k)}$, we denote the associated formal space germs to $A$, resp. $A_{i}$, resp. $A / \mathfrak{a}^{k+1}$. Let $\mathscr{F}$ be in $\operatorname{Coh}^{\prime}\left(X_{A}, 0\right)$. We take an $S$-embedding $X \hookrightarrow \bar{D}_{S}$, where $\bar{D}$ is a compact polydisc around 0 in some $\mathbf{C}^{n}$, further a free graded algebra $\mathscr{R}$ over $\mathscr{O}_{\bar{D}}$, such that $\mathscr{R}_{T}$ has a differential $s_{X_{T}}$, giving a resolvent of $\mathscr{O}_{X_{T}}$ (we may assume that $s_{X_{T}} \mid \mathscr{R}_{T}^{(\geq-1)}$ is already defined over $S$ ), and a free graded $\mathscr{R}$-module $\mathscr{M}=$ $\amalg_{i \in I}^{T} \mathscr{R} m_{i}$ together with an $s_{X_{T}}$-compatible differential $s$ on $\mathscr{M}_{T}$, such that $\left(\mathscr{M}_{T}, s\right)$ is a resolution of $\mathscr{F}$. We denote by $s_{0}: \mathscr{M} \rightarrow \mathscr{M}$ the fiber differential of $s$. By the formula $\delta\left(m_{i}\right):=1 \hat{\otimes} s_{0}\left(m_{i}\right), i \in I$, we get a $s_{X_{T}}$-compatible differential $\delta: \mathscr{M}_{T} \rightarrow \mathscr{M}_{T}$. We put

$$
\begin{aligned}
{ }^{\infty} \mathscr{L}_{T}:=\operatorname{Hom}_{\mathscr{R}_{T}}\left(\mathscr{M}_{T}, \mathscr{M}_{T}\right), & \mathscr{L}_{T}:=\left({ }^{\infty} \mathscr{L}_{T} / F^{2}\left(^{\infty} \mathscr{L}_{T}\right)\right)^{(\geq 0)}, \\
{ }^{\infty} \mathscr{L}:=\operatorname{Hom}_{\mathscr{R}}(\mathscr{M}, \mathscr{M}), & \mathscr{L}:=\left({ }^{\infty} \mathscr{L} / F^{2}\left({ }^{\infty} \mathscr{L}\right)\right)^{(\geq 0)},
\end{aligned}
$$

where $F^{m}(\cdots)$ is defined analogously to $(7.1)$. Then these graded objects are complexes (with differential induced by $u \mapsto[s, u]$, resp. $u \mapsto\left[s_{0}, u\right]$ ) and sheaves of graded Lie algebras. The homogeneous components of $\mathscr{L}_{T}$, resp. $\mathscr{L}$, are finite free modules over $\mathscr{O}_{\bar{D}_{T}}$, resp. $\mathscr{O}_{\bar{D}}$. We have

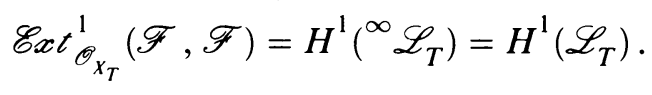


Here the last identity follows from the fact that $H^{\nu}\left(F^{2}\left(\mathscr{L}_{T}\right)\right)=0$ for $\nu=1,2$. Moreover, since $\operatorname{Sing}_{X_{T}}(\mathscr{F})$ is finite over $T$ and $\mathscr{F}$ is flat over $T$ at all points not in $\operatorname{Sing}_{X_{T}}(\mathscr{F})$, we obtain easily

$$
\operatorname{dim}_{\mathbf{C}} H^{1}(\mathscr{L})_{\{0\}}<\infty .
$$

Now the map

$$
q_{T}: \mathscr{L}_{T}^{1} \rightarrow \mathscr{L}_{T}^{2}
$$

induced by

$$
w \mapsto[\delta, w]+\frac{1}{2}[w, w]
$$

is even "definable over $S . "$ This follows immediately from the fact that $s_{X_{T}} \mid \mathscr{R}_{T}^{(\geq-1)}$ is defined over $S$ and $[\delta, w] \mid \mathscr{R}_{T}^{-2} \mathscr{M}_{T}^{0}=0$, so $[\delta,-] \mid \mathscr{L}_{T}^{1}$ is definable over $S$. We fix a triangle set $\Delta \subset \mathbf{R}_{>0}^{n}$ which is privileged for the complex $\mathscr{L}^{(0,2)}$ (compare [B-K, Kap. II, §3]). Then

$$
L_{\rho}:=\Gamma(D(\rho), \mathscr{L}),{ }^{10} \quad \rho \in \Delta,
$$

is a graded PO-Lie algebra (even in the "strict sense") with PO-Lie group $G_{\rho}$, associated to $L_{\rho}^{0}$, and we have restriction maps

$$
L_{\rho} \rightarrow L_{\rho^{\prime}}, \quad \text { for } \rho^{\prime} \leq \rho .
$$

We set

$$
\bar{L}_{\rho}:={\overline{\left(L_{\rho}\right)}}_{1 / 2},
$$

so $\bar{L}_{\rho}$ is a graded Banach Lie algebra, and we may assume that

$$
H^{1}\left(\bar{L}_{\rho}\right)=H^{1}(\mathscr{L})_{\{0\}}, \quad \rho \in \Delta,
$$

if $\Delta$ is small enough. The map (11.1.2) induces strictly convergent $S$-morphisms

and $S$-morphisms

$$
q_{\rho}: L_{\rho, S}^{1} \rightarrow L_{\rho, S}^{2}
$$

$$
\bar{q}_{\rho}: \bar{L}_{\rho, S}^{1} \rightarrow \bar{L}_{\rho, S}^{2}
$$

of banachanalytic spaces, compatible in $\rho$. We put $W_{\rho}:=\bar{q}_{\rho}^{-1}(\mathfrak{o})$, where $\mathfrak{o}$ is the zero-section of $\bar{L}_{\rho, S}^{2} \rightarrow S$. Again, we have the adjoint representation

$$
\omega_{\rho}: G_{\rho, S} \times{ }_{S} L_{\rho, S} \rightarrow L_{\rho, S},
$$

induced by $(g, u) \mapsto g u g^{-1}$ (if we use an "interpretation" of $G_{\rho}$ as automorphisms), and also

$$
\bar{\omega}_{\rho}: \bar{G}_{\rho, S} \times_{S} \bar{L}_{\rho, S} \rightarrow \bar{L}_{\rho, S} .
$$

\footnotetext{
${ }^{10}$ Here $D(\rho)$ is the open polydisc of multi-radius $\rho$ around $0 \in \mathbf{C}^{n}$.
} 
The Taylor expansion $\bar{\omega}_{\rho}^{1}[\delta]$ of $\bar{\omega}_{\rho}^{1}: \bar{G}_{\rho, S} \times{ }_{S} \bar{L}_{\rho, S}^{1} \rightarrow L_{\rho, S}^{1}$ "around $(0, \delta)$ " is well defined here: it is induced by $(g, u) \mapsto g u g^{-1}+g \delta g^{-1}-\delta$, and $\left(g \delta g^{-1}-\delta\right) \mid \mathscr{K}_{T}^{(\geq-2)}$ is already defined over $S$, since its restriction to $\mathscr{R}_{T}^{-2} \mathscr{M}_{T}^{0}$ is zero. In this way, we obtain again an operation

$$
\bar{\omega}_{\rho}^{1}[\delta]: \bar{G}_{\rho, S} \times{ }_{s} \bar{L}_{\rho, S}^{1} \rightarrow \bar{L}_{\rho, S}^{1}
$$

and $\bar{q}_{\rho}$ is equivariant with respect to $\bar{\omega}_{\rho}^{1}[\delta], \bar{\omega}_{\rho}^{2}$.

We fix $\rho_{0} \in \Delta$. By the proof of (3.3.2) and (11.1.1), (11.1.3), we can find a finite-dimensional subgerm $Y \subset\left(W_{\rho_{0}}, 0\right)$, such that

$$
\bar{\omega}_{\rho}^{1}[\delta]: \bar{G}_{\rho, S} \times{ }_{S} Y \rightarrow W_{\rho}
$$

is a smooth $S$-map of banachanalytic space germs for each $\rho \in \Delta, \rho \leq \rho_{0}$. Our differential $s$ gives a $S$-map

$$
\tilde{s}_{\rho}: T \rightarrow W_{\rho}
$$

for each such $\rho$, compatible with $W_{\rho} \rightarrow W_{\rho^{\prime}}$ for $\rho^{\prime} \leq \rho$. Let $(g, \chi): T \rightarrow$ $\bar{G}_{\rho, S} \times_{S} Y$ be a lifting of $\tilde{s}_{\rho}$. Then $\chi$ is already defined on some $T_{i}$. The construction of $\S 9$ shows us that there is a module $\mathscr{G} \in \operatorname{Coh}\left(X_{A_{i}}, 0\right)$ with $\mathscr{G}_{A} \cong \mathscr{F}$. It remains to show that $\mathscr{G}$ is in $\operatorname{Coh}^{\prime}\left(X_{A_{i}}, 0\right)$. Let $T_{i}^{\prime} \subset T_{i}$ be the universal subspace with the property that $\mathscr{G}_{T_{i}^{\prime}, x}$ is flat over $T_{i}^{\prime}$ for all points $x \in X_{0} \backslash\{0\}$. If the isomorphism $\mathscr{G}_{A} \stackrel{\sim}{\rightarrow} \mathscr{F}$ is defined at all points of $X_{0} \subset \bar{D}$ (otherwise shrink $D$ ), then $T \rightarrow T_{i}$ necessarily factorizes over $T_{i}^{\prime}$. But then $T_{i}=T_{i}^{\prime}$, since $A_{i} \rightarrow A$ is injective. This implies $\mathscr{G} \in \operatorname{Coh}^{\prime}\left(X_{A_{i}}, 0\right)$.

We consider the second functor in (1.9), which is the composition of

$$
\operatorname{Coh}^{\prime}\left(X_{A}, 0\right) \stackrel{\wedge}{\rightarrow} \operatorname{Coh}^{\prime}\left(\left(X_{A}, 0\right)^{\wedge}\right) \stackrel{\kappa}{\stackrel{\lim }{\leftrightarrows}} \operatorname{Coh}_{k}^{\prime}\left(X_{A / a^{k+1}}, 0\right),
$$

where "^" means the completion with respect to the ideal $\mathfrak{a} \mathscr{O}_{X_{A}, 0}$. If we replace $\operatorname{Coh}^{\prime}(\cdots)$ by $\operatorname{Coh}(\cdots)$, then it is known that $\kappa$ is an equivalence of categories. But this is also true for $\operatorname{Coh}^{\prime}(\cdots)$, which is a consequence of the following facts:

Let $A$ be a noetherian commutative ring and $M$ a finite $A$-module. Then the singularity set $\operatorname{Sing}_{A}(M)$ of $M$ can be defined by the ideal

$$
S_{A}(M):=\sum_{r=0}^{\infty} F_{r}(M) \cdot \operatorname{Ann}_{A}\left(F_{r-1}(M)\right)
$$

(compare [Ku, Appendix D]), where $F_{i}(M)$ denotes the $i$ th Fitting ideal of $M$.

(11.2) Lemma. Let $A \rightarrow B$ be a local homomorphism of local noetherian rings, $\mathfrak{a} \subset \mathfrak{m}_{A}$ an ideal, and $M$ a finite $B$-module. We put $A_{k}:=A / \mathfrak{a}^{k+1}, B_{k}:=$ $B \otimes_{A} A_{k}, M_{k}:=M \otimes_{B} B_{k}$. 
Assume that $B_{k} / S_{B_{k}}\left(M_{k}\right)$ is a finite $A_{k}$-module for each $k \in \mathbf{N}$. Then $B / S_{B}(M)+\mathfrak{a} B$ is a finite $A_{0}$-module. If $A$ is $\mathfrak{a}$-adically complete, then $B / S_{B}(M)$ is finite over $A$.

Proof. Take a prime ideal $\mathfrak{y} \in \operatorname{Spec}(B)$ with $\mathfrak{y} \supset \mathfrak{a} B$ and let $\mathfrak{y}_{k}$ be the image of $\mathfrak{y}$ in $B_{k}$, which is prime again. Then we have the equivalent properties

$$
\mathfrak{y} \supset S_{B}(M) \Leftrightarrow \exists k \in \mathbf{N}: \mathfrak{y}_{k} \supset S_{B_{k}}\left(M_{k}\right) .
$$

We denote by $S_{k}$ the pre-image of $S_{B_{k}}\left(M_{k}\right)$ in $B$. Then, obviously, $S_{B}(M) \subset$ $S_{k+1} \subset S_{k}$. So for any $\mathfrak{y} \in \operatorname{Spec}(B)$, we have

$$
\mathfrak{y} \supset S_{B}(M)+\mathfrak{a} B \Leftrightarrow \exists k: \mathfrak{y} \supset S_{k} .
$$

This implies $\sqrt{S_{B}(M)+\mathfrak{a} B}=\sqrt{S_{k}}$, for $k$ large enough. By assumption, $B / S_{k}$ is finite over $A_{k}$. So we get that $B / S_{B}(M)+\mathfrak{a} B$ is finite over $A_{0}$.

Now assume $A$ to be a-adically complete and set $N:=B / S_{B}(M)$. Obviously, it is sufficient to show that $(N)^{\wedge \mathfrak{a}}$ is finite over $A$, since $N \rightarrow(N)^{\wedge \mathfrak{a}}$ is injective. But this is standard now.

In the next step we are going to show the surjectivity of ${ }^{\wedge}$ which is more subtle. Fix $F$ in $\operatorname{Coh}^{\prime}\left(\left(X_{A}, 0\right)^{\wedge}\right)$. With the notations above, $F$ has a resolution of the form $\left(\mathscr{M}_{T} \mid\left(D_{T}, 0\right)^{\wedge}, s\right)$, where "^" means completion with respect to $\mathrm{a} \mathscr{O}_{D_{T}, 0}$. For abbreviation, we set $\widehat{M}_{T}:=\left(\mathscr{M}_{T} \mid\left(D_{T}, 0\right)\right)^{\wedge}, M:=\mathscr{M} \mid(D, 0)$, $R:=\mathscr{R} \mid(D, 0), \widehat{R}_{T}:=\left(\mathscr{R}_{T} \mid\left(D_{T}, 0\right)\right)^{\wedge \mathfrak{a}}, B:=\mathscr{O}_{X_{T}, 0}, \widehat{B}:=\widehat{B}^{\mathfrak{a}}, \bar{B}:=B / \mathfrak{m}_{A} B$, and $K:=\mathscr{O}_{D, 0}=R^{0}$. Moreover, we define ${ }^{\infty} L_{T}:=\operatorname{Hom}_{\widehat{R}_{T}}\left(\widehat{M}_{T}, \widehat{M}_{T}\right)$ and $L_{T}, L$ similarly as in the beginning. Obviously, we have $H^{1}\left({ }^{\infty} L_{T}\right)=H^{1}\left(L_{T}\right)$ and $L_{T} / \mathfrak{m}_{T} L_{T}=L$. We want to show that $\operatorname{supp} H^{1}(L) \subset\left\{\mathfrak{m}_{K}\right\}$. For this, let $\mathfrak{y} \in \operatorname{Spec}(K), \mathfrak{y} \neq \mathfrak{m}_{K}$, and $\mathfrak{p}$ be the pre-image of $\mathfrak{y}$ in $\widehat{R}_{T}^{0}$. We distinguish two cases:

$$
\mathfrak{p} \not \supset \operatorname{Ker}\left(\widehat{R}_{T}^{0} \rightarrow \widehat{B}\right) .
$$

Here we get $F_{\mathrm{p}}=0$. Consequently, $\left(\widehat{M}_{T}\right)_{\mathfrak{p}}$ and $(M)_{\mathfrak{y}}$ are homotopic to zero. This immediately implies $H^{1}(L)_{\mathfrak{y}}=H^{1}\left(L_{\mathfrak{\eta}}\right)=0$.

$$
\mathfrak{p} \supset \operatorname{Ker}\left(\widehat{R}_{T}^{0} \rightarrow \widehat{B}\right) \text {. }
$$

Let $\mathfrak{p}^{\prime}$, resp. $\mathfrak{y}^{\prime}$, be the image of $\mathfrak{p}$, resp. $\mathfrak{y}$, in $\widehat{B}$, resp. $\bar{B}$. Then $\mathfrak{p}^{\prime}$ and $\mathfrak{y}^{\prime}$ are prime again, since $\mathfrak{y} \supset \operatorname{Ker}\left(R^{0} \rightarrow \bar{B}\right)$. We assume $\mathfrak{p}^{\prime} \supset S_{\widehat{B}}(F)$. Then $\mathfrak{y}^{\prime} \supset S_{\widehat{B}}(F) \bar{B}$. By assumption, $\widehat{B} / S_{B}(F)$ is a finite $A$-module, so $\mathfrak{y}^{\prime} \supset \mathfrak{m}_{\bar{B}} \frac{k}{}$ for $k$ large enough. This also implies $\mathfrak{y} \supset \mathfrak{m}_{K}^{k}$ and so $\mathfrak{y}=\mathfrak{m}_{K}$, which is a contradiction. Consequently, $\mathfrak{p}^{\prime} \not \supset S_{\widehat{B}}(F)$ and $F_{\mathfrak{p}^{\prime}}$ is free over $\widehat{B}_{\mathfrak{p}^{\prime}}$. By the flatness of the composition $A \rightarrow B \rightarrow \widehat{B} \rightarrow \widehat{B}_{\mathfrak{p}^{\prime}}$, we see that $M_{\mathfrak{y}}$ is a resolution 
of $\bar{F}_{\mathfrak{y}^{\prime}}$. This implies again $H^{1}\left(L_{\mathfrak{y}}\right)=H^{1}\left(\operatorname{Hom}_{R_{\mathfrak{n}}}\left(M_{\mathfrak{y}}, M_{\mathfrak{y}}\right)\right)$. Using the quasiisomorphisms

$$
\left.\operatorname{Hom}_{\left(\widehat{R}_{T}\right)_{\mathfrak{p}}}\left(\left(\widehat{M}_{T}\right)_{\mathfrak{p}}, \bar{F}_{\mathfrak{y}^{\prime}}\right) \longrightarrow M_{\mathfrak{y}}, \longrightarrow M_{\mathfrak{y}}\right) \longrightarrow \operatorname{Hom}_{R_{\mathfrak{n}}}\left(M_{\mathfrak{y}}, \bar{F}_{\mathfrak{y}^{\prime}}\right)
$$

we get $H^{1}\left(L_{\mathfrak{y}}\right)=\operatorname{Ext}_{\widehat{B}_{\mathfrak{p}^{\prime}}}^{1}\left(F_{\mathfrak{p}^{\prime}}, \bar{F}_{\mathfrak{y}^{\prime}}\right)=0$.

The finiteness condition (11.1.1) holds now in this context too. With the same argument as above, $s$ induces $S$-morphisms

$$
s^{(k)}: T^{(k)} \rightarrow W_{\rho(k)}, \quad \rho(k) \in \Delta,
$$

for each $k \in \mathbf{N}$. Observe that the sequence $(\rho(k))_{k \in \mathbf{N}}$ may converge to $0 \in \mathbf{R}^{n}$. By (11.1.4), we have liftings $\left(g_{\rho(k)}, \chi_{k}\right)$ of $s^{(k)}$ to $\bar{G}_{\rho(k), S} \times{ }_{S} Y$, which can be even chosen compatible in $k$ (use induction on $k$ and the smoothness in (11.1.4)). Hence, we obtain a $S$-map $\chi: T \rightarrow Y$ with $\bar{\omega}_{\rho(k)}^{1}[\delta]\left(g_{\rho(k)}, \chi\right)=s^{(k)}$ for all $k$. Because of $Y \subset W_{\rho_{0}}$, we get a module $\mathscr{G}$ in $\operatorname{Coh}\left(X_{A}, 0\right)$, such that $\hat{\mathscr{G}} \cong F$. Note that $\mathscr{G}$ is defined over $D\left(\rho_{0}\right)_{A}$. But we have also $\mathscr{G} \in$ $\operatorname{Coh}^{\prime}\left(X_{A}, 0\right)$, since $S_{B}(\mathscr{G})^{\wedge \mathfrak{a}}=S_{\widehat{B}}(\hat{\mathscr{G}})$.

The proof of Theorem (1.10) uses the same method and techniques, therefore we restrict ourselves here to mentioning the data: Take $\mathscr{X}$ in $\operatorname{Gan}^{\prime}(A)$, a $T$ embedding $\mathscr{X} \hookrightarrow \bar{D}_{T}$ (where $\bar{D}$ is some compact polydisc), a resolvent $\mathscr{R}_{T}$ of $\mathscr{O}_{\mathscr{Z}}$ and put

$$
\begin{array}{rlrl}
{ }^{\infty} \mathscr{L}_{A}:=\operatorname{Der}_{T}\left(\mathscr{R}_{T}, \mathscr{R}_{T}\right), & & \mathscr{L}_{A}:=\left({ }^{\infty} \mathscr{L}_{A} / F^{2}\left({ }^{\infty} \mathscr{L}_{A}\right)\right)^{(\geq 0)}, \\
{ }^{\infty} \mathscr{L}:=\operatorname{Der}(\mathscr{R}, \mathscr{R}), & \mathscr{L}:=\left({ }^{\infty} \mathscr{L} / F^{2}\left({ }^{\infty} \mathscr{L}\right)\right)^{(\geq 0)} .
\end{array}
$$

The assumption on the smoothness locus of $\mathscr{X} \rightarrow T$ gives the finiteness condition (11.1.1). Observe that $\operatorname{Der}_{T}\left(\mathscr{R}_{T}, \mathscr{R}_{T}\right)=\operatorname{Hom}_{\mathscr{R}_{T}}\left(\Omega_{\mathscr{R}_{T} / T}, \mathscr{R}_{T}\right)$ and $\operatorname{Der}(\mathscr{R}, \mathscr{R})=\operatorname{Hom}_{\mathscr{R}}\left(\Omega_{\mathscr{R}}, \mathscr{R}\right)$. One chooses $\Delta$ again privileged for $\mathscr{L}^{(0,2)}$. The rest of the proof of the assertion can be taken from $\S 8$ and from above.

\section{SOME APPLICATIONS}

(12.1) First we want to give a proof for Corollary (1.3). Obviously, it is sufficient to show the surjectivity of the functor in (1.3). For this, let $\mathscr{F}$ be in $\operatorname{Vect}\left(X_{A}\right)$ of rank $r$ (we may assume that the special fiber $X_{0}$ is connected) and $\mathscr{G}$ a coherent sheaf in $\operatorname{Coh}\left(X_{A_{i}}\right)$ with $\mathscr{G}_{A} \cong \mathscr{F}$. Let $Y^{0} \supset Y^{1} \supset \cdots \supset$ $Y^{k}$ be the chain of closed subspaces of $X_{A_{i}}$, defined by the chain of Fitting ideals $F_{0}(\mathscr{G}) \subset F_{1}(\mathscr{G}) \subset \cdots \subset F_{k}(\mathscr{G})$ of $\mathscr{G}$ (see [Ku, appendix D]). Because of $F_{\nu}(\mathscr{G})_{A}=F_{\nu}\left(\mathscr{G}_{A}\right)$ for all $\nu$, we get $X_{A}=Y_{A}^{0}=Y_{A}^{1}=\cdots=Y_{A}^{r-1} \supset Y_{A}^{r}=\varnothing$. 
The fully faithfulness of (1.1.2) implies that $X_{A_{j}}=Y_{A_{j}}^{\nu}$ for $\nu=0, \ldots, r-1$ and $Y_{A_{j}}^{r}=\varnothing$, where $j$ is some index $\geq i$. Then $\mathscr{G}_{A_{j}}$ must be locally free of rank $r$ (see [loc. cit., D.13]). This shows the assertion.

Let $X, S, A, A_{i}$ be as in (1.2) and $\mathscr{E}$ a coherent $\mathscr{O}_{X}$-module. For a formal $\mathscr{O}_{S}$-algebra $B$, we define

$$
Q_{\mathscr{E} / X / S, p}(B)
$$

to be the set of all quotients $\mathscr{F}$ of $\mathscr{E}_{B}$ in $\operatorname{Coh}_{p}\left(X_{B}\right)$. Obviously, $Q_{\mathscr{E} / X / S, p}(-)$ is a (covariant) functor. Theorem (1.2) immediately gives us

(12.2) Theorem. The canonical map

$$
\underset{i \in I}{\lim _{\mathscr{E} / X / S, p}} Q_{i}\left(A_{i}\right) \rightarrow Q_{\mathscr{E} / X / S, p}(A)
$$

is bijective.

(12.3) Remark. Using Frisch's theorem on the universal flat locus (compare [Fr] or also [Fl-Ko, Theorem (5.7)]), it follows from (1.2), resp. (1.7), that the functor (1.1.3), resp. (1.4.2), is an equivalence of categories. If we replace $Q_{\mathscr{E} / X / S, p}$ by the subfunctor $Q_{\mathscr{E} / X / S, p, f}$, consisting of those quotients which are in addition flat over the base, then Theorem (12.2) holds for $Q_{\mathscr{E} / X / S, p, f}$ as well (using the same argument).

In the book [B-K], the existence of semiuniversal deformations for 1-convex spaces, considered as a germ along its exceptional set $E$, has been proved under the assumption that $E$ is analytically thin (see [loc. cit., (V.5.2)(b)]). We are going to show that this condition is not necessary by using the surjectivity of (1.1.2). The procedure is nearly the same as in [loc. cit.], the only difference is that one has to prove a stronger version of the formal principle (V.6.2) for deformations.

(12.4) Let $S$ be a complex space and $T \subset S$ a closed complex subspace with infinitesimal neighborhoods $S_{n}, n \in \mathbf{N}$. Moreover, we fix a point $s \in T$. For a complex space $X$ over $S$ and a closed subset $K$ of the fiber $X_{s}$, we denote by $(X / K)^{\wedge}$ the completion of the pseudocomplex space $X \mid K=\left(K, \mathscr{O}_{X} \mid K\right)$ along (the pre-image of) $T$. If $Y$ is another complex space over $S, L$ a closed subset of $Y_{s}$, and $f: X \rightarrow Y$ a $S$-morphism with $f(K) \subset L$, then the completion

$$
(f \mid(K, L))^{\wedge}: X|K \rightarrow Y| L
$$

of

$$
(f \mid(K, L)): X|K \rightarrow Y| L
$$

along $T$ is well defined. With these notions we have

(12.5) Theorem. Let $X \rightarrow S, X^{\prime} \rightarrow S$ be 1-convex spaces over $S$ with relative exceptional sets $E, E^{\prime}$ and $\bar{\varphi}:\left(X \mid E_{s}\right)^{\wedge} \rightarrow\left(X^{\prime} \mid E_{s}^{\prime}\right)^{\wedge}$ an $S$-morphism. Then 
for each integer $n \geq 0$, there is an $S$-morphism $\varphi: X\left|E_{s} \rightarrow X^{\prime}\right| E_{s}^{\prime}$ such that $\varphi_{S_{n}}=\bar{\varphi}_{S_{n}}$.

Proof. It is no restriction to assume $E_{s}$ and $E_{s}^{\prime}$ connected. Denote by $f: X \rightarrow$ $Y, f^{\prime}: X^{\prime} \rightarrow Y^{\prime}$ the relative Remmert quotients of $X, X^{\prime}$ over $S$ and put $F:=f(E), F^{\prime}:=f^{\prime}\left(E^{\prime}\right)$. Then there is exactly one $\widehat{S}$-morphism $\bar{\psi}:\left(Y \mid F_{s}\right)^{\wedge} \rightarrow$ $\left(Y^{\prime} \mid F_{s}^{\prime}\right)^{\wedge}$, making the diagram

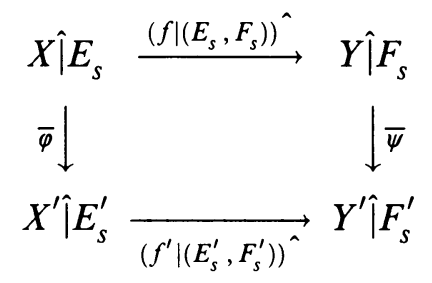

commutative. Since $E_{s}, E_{s}^{\prime}$ are connected, one has $F_{s}=\{y\}, F_{s}^{\prime}=\left\{y^{\prime}\right\}$ and the assertion follows now from (12.7).

(12.6) We fix the situation in (12.4). Let $f: X \rightarrow Y, f^{\prime}: X^{\prime} \rightarrow Y^{\prime}$ be two separated $S$-morphisms and $L=\{y\}, L^{\prime}=\left\{y^{\prime}\right\}$ be points in $Y_{s}, Y_{s}^{\prime}$. We assume that $K=f^{-1}(L), K^{\prime}=f^{\prime-1}\left(L^{\prime}\right)$ and $\bar{\varphi}:(X \mid K)^{\wedge} \rightarrow\left(X^{\prime} \mid K^{\prime}\right)^{\wedge}, \bar{\psi}:(Y \mid L)^{\wedge} \rightarrow$ $\left(Y^{\prime} \mid L^{\prime}\right)^{\wedge}$ are $\widehat{S}$-morphisms, such that the diagram

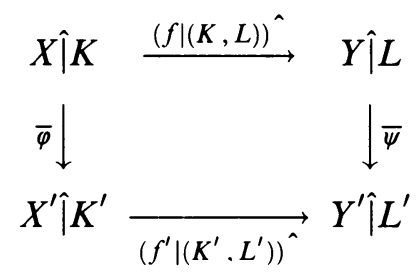

is commutative.

(12.7) Theorem. Assume that $f$ is proper in the situation above. Then for each $n \geq 0$, there are $S$-morphisms $\varphi: X\left|K \rightarrow X^{\prime}\right| K^{\prime}, \psi: Y\left|L \rightarrow Y^{\prime}\right| L^{\prime}$ with $\varphi_{S_{n}}=$ $\bar{\varphi}_{S_{n}}, \psi_{S_{n}}=\bar{\psi}_{S_{n}}$ and such that

$$
\left(f^{\prime} \mid\left(K^{\prime}, L^{\prime}\right)\right) \circ \varphi=\psi \circ(f \mid(K, L)) .
$$

Proof. In the following, we consider $X, X^{\prime}$, resp. $Y, Y^{\prime}$, always as pseudocomplex spaces along $K, K^{\prime}$, resp. $L, L^{\prime}$. Obviously, $A:=\left(\mathscr{O}_{Y}\right)^{\wedge}=\mathscr{O}_{\widehat{Y}}$ (completion with respect to $T$ ) is a formal algebra over $\mathscr{O}_{Y}$. We fix an exhaustion $\left(A_{i}\right)_{i \in I}$ of $A$ by convergent $\mathscr{O}_{Y}$-subalgebras. Let $T_{i}$ be the associated space germ to $A_{i}$. Then there is a factorization of $\bar{\psi}$ into $\widehat{Y} \rightarrow T_{i} \rightarrow Y^{\prime}$ for some $i \in I$ and

$$
Z:=X_{T_{i}} \times{ }_{T_{i}} X_{T_{i}}^{\prime}
$$

is a space in $\operatorname{An}\left(T_{i}\right)$. Obviously, we have $Z_{\widehat{Y}}=\widehat{X} \times_{Y^{\prime}} X^{\prime}$. The graph $\Gamma_{\bar{\varphi}}$ of $\bar{\varphi}$ is a closed subspace of $Z_{\widehat{Y}}$, proper over $\widehat{Y}$. By (1.2), there is some $j \geq i$ and a closed subspace $\Gamma$ of $Z_{T_{j}}$ with $\Gamma_{\widehat{Y}}=\Gamma_{\bar{\varphi}}$. We may also assume that 
the projection $\Gamma \rightarrow X_{T_{j}}$ is an isomorphism, otherwise we increase $j$ enough. Consequently, $\Gamma$ is the graph of a $T_{j}$-morphism $\chi: X_{T_{j}} \rightarrow X_{T_{j}}^{\prime}$. Now, we take an $n$th order approximation $\rho: Y \rightarrow T_{j}$ of the $Y$-morphism $\widehat{Y} \rightarrow T_{j}$, such that $Y \stackrel{\rho}{\rightarrow} T_{j} \rightarrow Y$ is the identity and put

$$
\begin{gathered}
\psi: Y \stackrel{\rho}{\rightarrow} T_{j} \rightarrow Y^{\prime} \\
\varphi: X=\left(X_{T_{j}} \stackrel{\rho}{\times_{T_{j}}} Y\right) \stackrel{\mathrm{pr}}{\longrightarrow} X_{T_{j}} \stackrel{\chi}{\rightarrow} X_{T_{j}}^{\prime} \stackrel{\mathrm{pr}}{\longrightarrow} X^{\prime} .
\end{gathered}
$$

Then $(\varphi, \psi)$ has the desired properties.

\section{APPENDIX}

For the convenience of the reader we want to mention here some notions from [B-K] which are indispensable for this paper.

Let $E$ be a $\mathrm{C}$-vector space. Then $E$ is called a $P O$-space if $E$ is endowed with a family $\left.\|\cdot\|_{\lambda}, \lambda \in\right] 0,1\left[\right.$, of seminorms, such that $\|\cdot\|_{\lambda^{\prime}} \leq\|\cdot\|_{\lambda}$ holds for $\lambda^{\prime} \leq \lambda$. Fix $\left.\varepsilon \in\right] 0,1\left[\right.$. The category $\mathrm{PO}_{\varepsilon}$ is by definition the category whose objects are PO-spaces and whose morphisms $u: E \rightarrow F$ are $\mathrm{C}$-linear mappings, such that there is a constant $C=C(u)>0$ with $\|u(e)\|_{\lambda} \leq C\|e\|_{\lambda}$ for all $e \in E$ and $\lambda \in\left[1-\varepsilon, 1\left[\right.\right.$. We put $\mathrm{PO}:=\lim _{\varepsilon} \mathrm{PO}_{\varepsilon}$. Let $E$ be a PO-space. Then $\bar{E}_{\lambda}$ denotes the completion of $E$ with respect to the seminorm $\|\cdot\|_{\lambda}$. The projective limit $E_{t}:=\lim _{\lambda<t} \bar{E}_{\lambda}$ of Banach spaces, defined for $\left.t \in\right] 0,1[$, is in a natural way a PO-space with $\|\cdot\|_{\lambda}^{t}:=\|\cdot\|_{t \lambda}$. For $0<t^{\prime} \leq t \leq 1$, one has canonical homomorphisms (also called "restrictions") $i_{t^{\prime}, t}: E_{t} \rightarrow E_{t^{\prime}}$ which are compatible with the PO-structures. Obviously, $E_{1}$ is the completion $\widehat{E}$ of $E$ as a topological vector space. $E$ is called nuclear if the canonical maps $\bar{E}_{\lambda} \rightarrow \bar{E}_{\lambda^{\prime}}$ are nuclear maps between Banach spaces for $\lambda^{\prime}<\lambda$. In this case all $E_{t}, 0<t \leq 1$, are FN-spaces. Let $\varepsilon$ be in $\left.] 0,1\right]$. We call $E \varepsilon$-good, if $E$ is complete and all maps $\bar{E}_{\lambda} \rightarrow \bar{E}_{\lambda^{\prime}}$ are injective for $1-\varepsilon<\lambda^{\prime} \leq \lambda<1$.

The most important example of a PO-space is the following. Let $x$ be a fixed point in $\mathbf{C}^{n}$ and

$$
P:=\left\{z \in \mathbf{C}^{n}:\left|z_{i}-x_{i}\right|<b_{i}, 1 \leq i \leq n\right\}
$$

the open polydisc around $x$ with multi-radius $b=\left(b_{1}, \ldots, b_{n}\right) \in\left(\mathbf{R}_{>0}\right)^{n}$. We denote by $P^{(\lambda)}$ the (concentric) shrinking of $P$ by the factor $\left.\lambda \in\right] 0,1[$. Let $f=\sum_{\nu} f_{\nu}(z-x)^{\nu}$ be a holomorphic function on $P$. We define

$$
\left.\|f\|_{\lambda}:=\sum_{\nu \in \mathbf{N}^{n}}\left|f_{\nu}\right| \lambda^{|\nu|} b^{\nu}, \quad \lambda \in\right] 0,1[.
$$

Then $E:=\Gamma\left(P, \mathscr{O}_{\mathbf{C}^{n}}\right)$ is a PO-space with this family of seminorms. Moreover, 
$E$ is 1 -good and nuclear. We have the identifications

$$
\begin{aligned}
\bar{E}_{\lambda} & =B\left(\overline{P^{(\lambda)}}, \mathscr{O}_{\mathbf{C}^{n}}\right), & & 0<\lambda<1, \\
E_{t} & =\Gamma\left(P^{(t)}, \mathscr{O}_{\mathbf{C}^{n}}\right), & & 0<t<1 .
\end{aligned}
$$

Here $B\left(K, \mathscr{O}_{\mathbf{C}^{n}}\right)$ denotes the Banach algebra of continuous functions $f: K \rightarrow \mathbf{C}$ on $K \subset \mathbf{C}^{n}$ compact, such that $f \mid \stackrel{\circ}{K}$ is holomorphic.

\section{REFERENCES}

[Art] M. Artin, Algebraization of formal moduli. I, Global Analysis (Papers in Honor of K. Kodaira), Princeton Univ. Press, Princeton, N.J., 1969, pp. 21-71.

[Bi $\left.{ }_{1}\right] \quad J$. Bingener, Über formale komplexe Räume, Manuscripta Math. 24 (1978), 253-293.

$\left[\mathrm{Bi}_{2}\right] \_$, Darstellbarkeitskriterien für analytische Funktoren, Ann. Sci. École Norm. Sup. 13 (1980), 317-347.

$\left[\mathrm{Bi}_{3}\right] \quad-$, Offenheit der Versalität in der analytischen Geometrie, Math. Z. 173 (1980), 241-281.

[B-K] J. Bingener and S. Kosarew, Lokale Modulräume in der analytischen Geometrie, Aspects of Math., D2, D3, Vieweg-Verlag, Braunschweig, 1987.

[Dou $\left.{ }_{1}\right]$ A. Douady, Le probleme des modules pour les sous-espaces analytiques compacts d'un espace analytique donné, Ann. Inst. Fourier (Grenoble) 16 (1966), 1-95.

$\left[\mathrm{Dou}_{2}\right] \ldots$ Le problème des modules locaux pour les espaces $\mathbf{C}$-analytiques compacts, Ann. Sci. École Norm. Sup. 7 (1974), 569-602.

[Fl-Ko] H. Flenner and S. Kosarew, On locally trivial deformations, Publ. Res. Inst. Math. Sci. 23 (1987), 627-665.

[Fo-Kn] O. Forster and K. Knorr, Konstruktion verseller Familien kompakter komplexer Räume, Lecture Notes in Math., vol. 705, Springer-Verlag, Berlin-Heidelberg-New York, 1979.

[Fr] J. Frisch, Aplatissement en géométrie analytique, Ann. Sci. École Norm. Sup. 1 (1968), 305-312.

[EGA] A. Grothendieck and J. Dieudonné, Éléments de géométrie algébrique, Inst. Hautes Études Sci. Publ. Math. 4, 8, 11, 17, 20, 24, 28, 32 (1960-1967).

[Knu] D. Knutson, Algebraic spaces, Lecture Notes in Math., vol. 203, Springer-Verlag, BerlinHeidelberg-New York, 1971.

[Ku] E. Kunz, Kähler differentials, Adv. Lectures Math., Vieweg-Verlag, Braunschweig, 1986.

$\left[\mathrm{Pa}_{1}\right] \quad$ V. P. Palamodov, Deformations of complex spaces, Russian Math. Surveys 31 (1976), 129197.

$\left[\mathrm{Pa}_{2}\right] \quad$, The tangent complex of an analytic space, Amer. Math. Soc. Transl. 122 (1984), 119-171.

[Pou] G. Pourcin, Théorème de Douady au-dessus de $S$, Ann. Scuola Norm. Sup. Pisa Cl. Sci. (4) 23 (1969), 451-459.

[S-T] Y. T. Siu and G. Trautmann, Deformations of coherent analytic sheaves with compact supports, Mem. Amer. Math. Soc. No. 238 (1981).

Université de Grenoble I, Institut Fourier, Laboratoire de Mathematiques, associe au CNRS, B.P. 74, 38402 Saint-Martin-d'Hères Cedex, France 